\title{
Rateless Coding for Wireless Relay Networks
}

\author{
By \\ Hulaiyel Al Khaledi
}

A thesis submitted to the Faculty of Graduate and Postdoctoral Affairs in partial fulfillment

of the requirements for the degree of

\author{
Master of Applied Science
}

Ottawa-Carleton Institute for Electrical and Computer Engineering

Department of Systems \& Computer Engineering

Carleton University

Ottawa, Ontario, Canada

July 2010

(C) 2010 Hulaiyel Al Khaledi 
Library and Archives

Canada

Published Heritage

Branch

395 Wellington Street Ottawa ON K1A ON4 Canada
Bibliothèque et

Archives Canada

Direction du

Patrimoine de l'édition

395 , rue Wellington Ottawa ON K1A ON4

Canada
Your file Votre référence

ISBN: 978-0-494-71508-6

Our file Notre référence

ISBN: 978-0-494-71508-6
NOTICE:

The author has granted a nonexclusive license allowing Library and Archives Canada to reproduce, publish, archive, preserve, conserve, communicate to the public by telecommunication or on the Internet, loan, distribute and sell theses worldwide, for commercial or noncommercial purposes, in microform, paper, electronic and/or any other formats.

The author retains copyright ownership and moral rights in this thesis. Neither the thesis nor substantial extracts from it may be printed or otherwise reproduced without the author's permission.
AVIS:

L'auteur a accordé une licence non exclusive permettant à la Bibliothèque et Archives Canada de reproduire, publier, archiver, sauvegarder, conserver, transmettre au public par télécommunication ou par l'Internet, prêter, distribuer et vendre des thèses partout dans le monde, à des fins commerciales ou autres, sur support microforme, papier, électronique et/ou autres formats.

L'auteur conserve la propriété du droit d'auteur et des droits moraux qui protège cette thèse. $\mathrm{Ni}$ la thèse ni des extraits substantiels de celle-ci ne doivent être imprimés ou autrement reproduits sans son autorisation.
In compliance with the Canadian Privacy Act some supporting forms may have been removed from this thesis.

While these forms may be included in the document page count, their removal does not represent any loss of content from the thesis.
Conformément à la loi canadienne sur la protection de la vie privée, quelques formulaires secondaires ont été enlevés de cette thèse.

Bien que ces formulaires aient inclus dans la pagination, il n'y aura aucun contenu manquant.

\section{Canadä}




\section{Abstract}

The importance of relaying has arisen lately as a cost effective technique for extending the coverage of wireless networks. In this thesis, we explore the application of rateless coding to relay networks. The rateless code applied here is a rate compatible code based on a standard QC-LDPC code. The throughput performance for three TDD relaying methods, named startover, combine and continue, is examined. It is shown that continue relaying is the best of the three relaying techniques, with least complexity. The theoretical throughput performance of linear relaying is derived and validated using simulations. The thesis generalizes the findings to triangular relay networks. Continue relaying is applied to a single cell wireless system, to evaluate how much it can enhance the performance of such a practical system against Additive White Gaussian Noise (AWGN), pathloss and shadowing. Rateless continue-relaying can vastly improve the throughput performance and reduce the likelihood of outage events. 


\section{Acknowledgments}

In the first place, I would like to thank God for giving me the opportunity to do my Master degree in Carleton University, with all the knowledgeable and professional people. My sincere gratitude goes to Professor lan D. Marsland for his supervision and guidance. His patience and support are endless and his teaching style and advisory skills are unique. I am indebted to him for finishing this work.

I gratefully acknowledge Professor Radford M. Neal from University of Toronto for sharing his software work of Low Density Parity Check Codes. His computer program was very essential to my simulation software.

Many thanks to the Faculty of Graduate Studies and Research, and the Department of Systems and Computer Engineering for the teaching quality and the professional environment they provide.

I convey special acknowledgments to my work supervisor Adham Mutairi, and Division Head Ali Masari for their support and encouragements.

Special thanks go to my mother and the memory of my father, who raised me with love and patience. No words can express my appreciation and love to them. Their support and prayers were the secrets of my success. I also would like to thank all my brothers and sisters for supporting and encouraging me.

I would like to thank my wife for inspiring me and supporting me at all times. Special thanks go to my children Yafa, Muhammad and Selman who make my life a joy.

Finally, I would like to thank everybody who helped me in this work and apologize to those I have not mentioned their names here. 


\section{Contents}

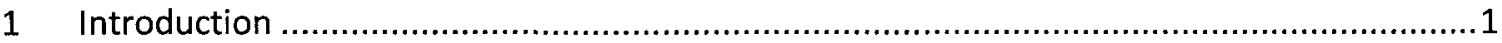

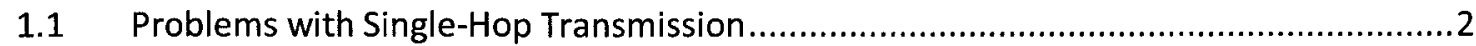

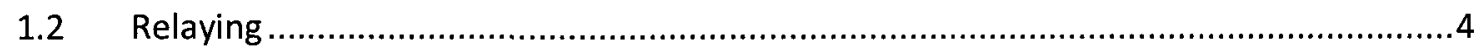

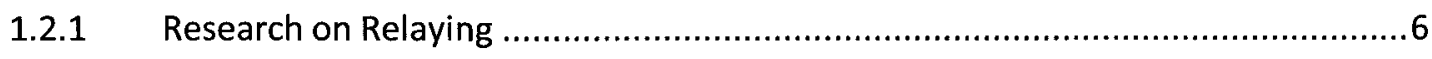

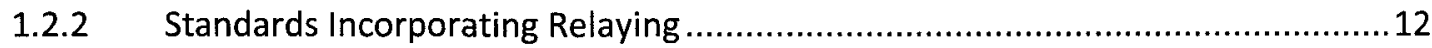

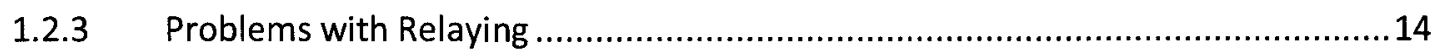

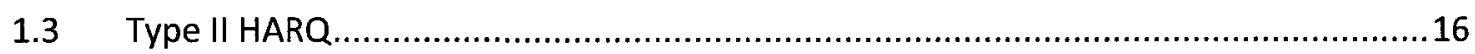

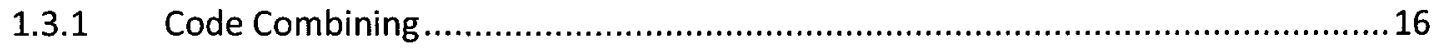

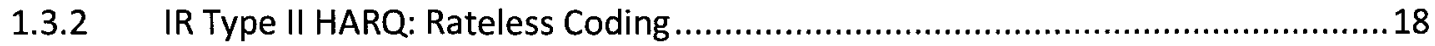

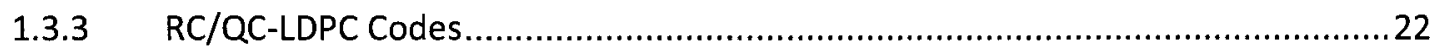

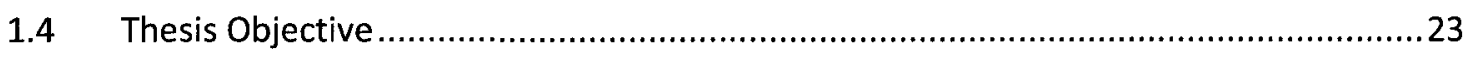

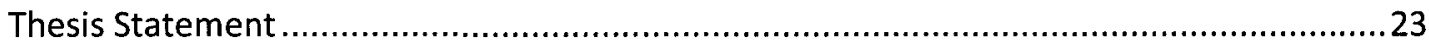

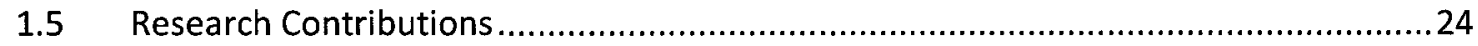

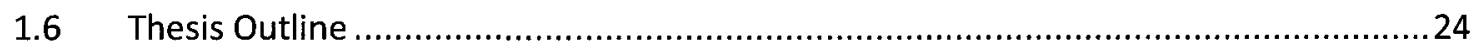

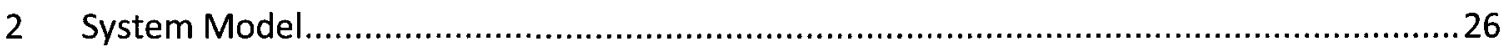

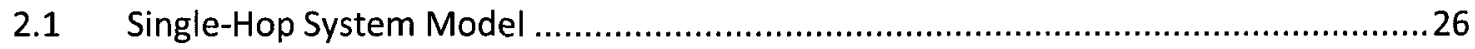

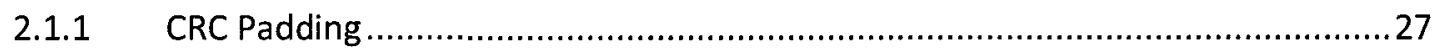

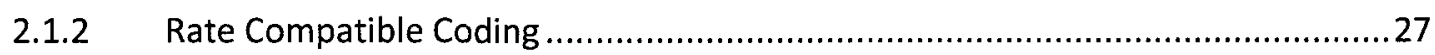

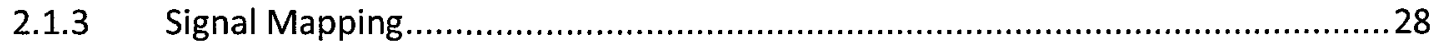

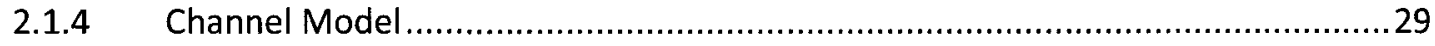

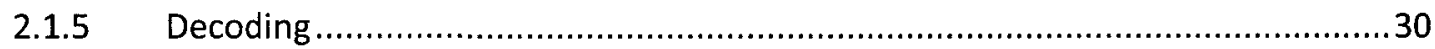

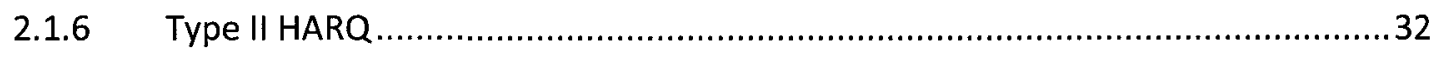

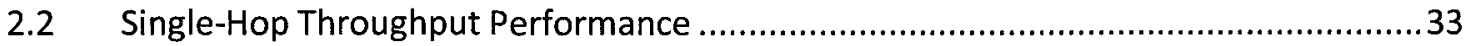

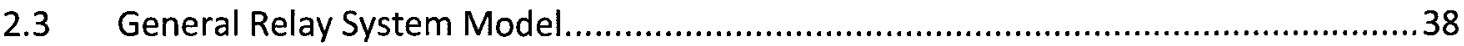

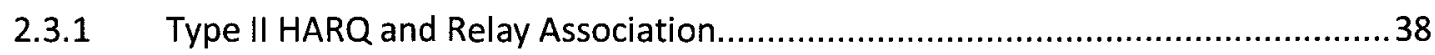

2.3.2 General Relay System Operation and Relaying Modes ....................................41

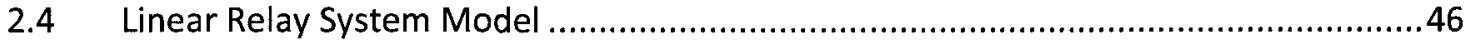

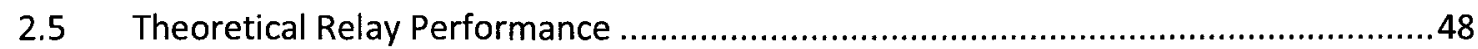

2.6 Generalization of Results to Triangular Relaying.................................................53

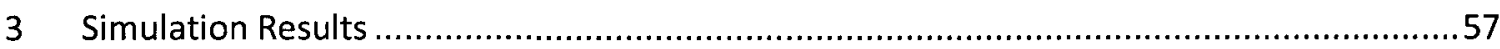




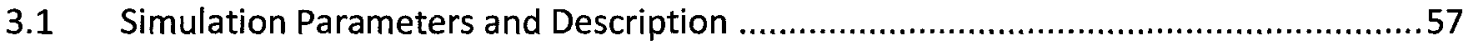

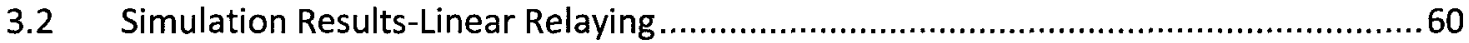

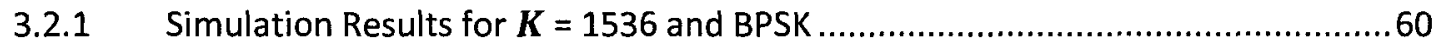

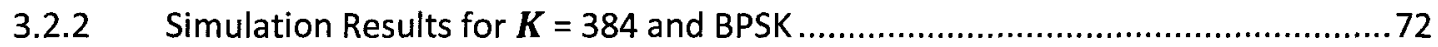

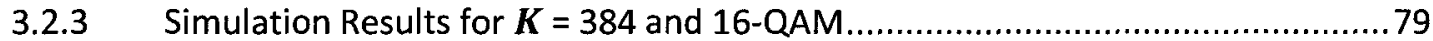

3.2.4 Maximum Relaying Throughput of Startover and Continue................................86

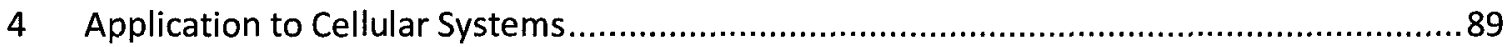

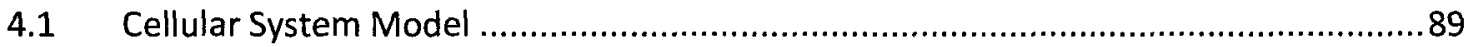

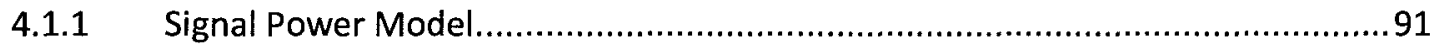

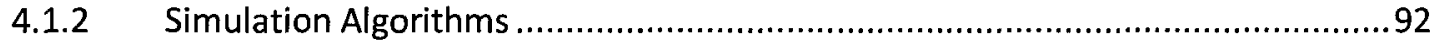

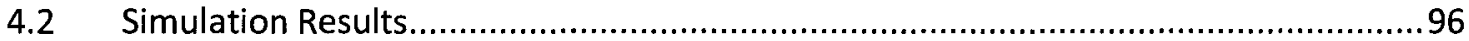

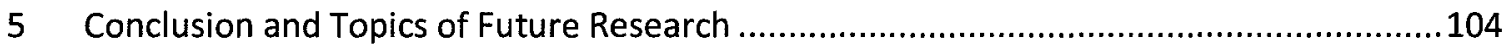

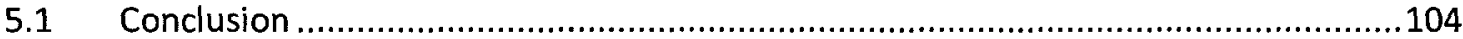

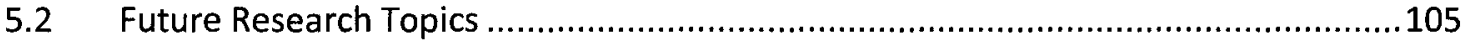

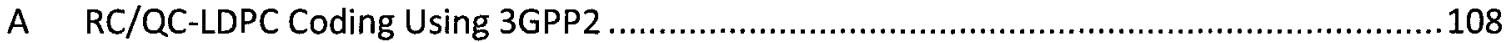

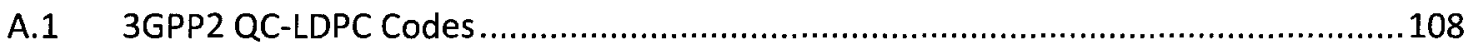

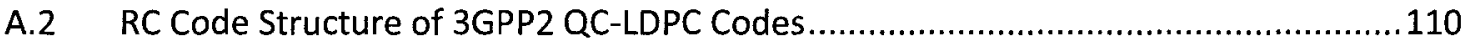

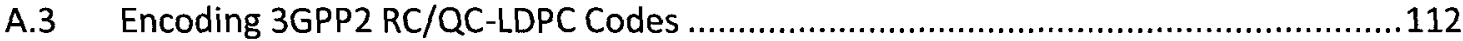

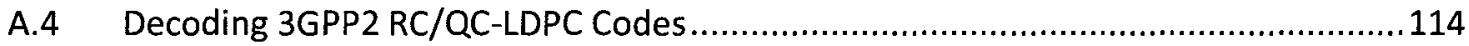

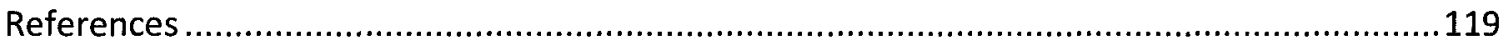




\section{List of Tables}

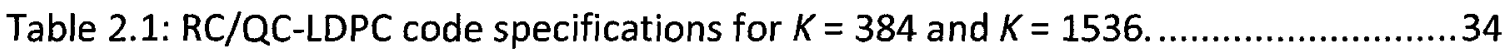

Table 2.2: SNR gap of the RC/QC-LDPC code from the channel capacities.....................38

Table 3.1: Linear relay model simulation parameters. .............................................59

Table 4.1: Simulation parameters for the one cell relay model. ................................96 


\section{List of Figures}

Figure 2.1: Signal constellation for (a) BPSK and (b) 16-QAM.

Figure 2.2: RC/QC-LDPC code throughput performance over AWGN channel (BPSK, $K=$

384)

Figure 2.3: RC/QC-LDPC code throughput performance over AWGN channel (16-QAM, K

$=384)$. 35

Figure 2.4: RC/QC-LDPC code throughput performance over AWGN channel (BPSK, $K=$ 1536). 36

Figure 2.5: RC/QC-LDPC code throughput performance over AWGN channel (16-QAM,

$\mathrm{K}=1536)$.

Figure 2.6: The formats of type II HARQ acknowledgment messages and Relay Association Message. 39

Figure 2.7: Message flow diagram of type II HARQ applied to relayed-network............40

Figure 2.8: General relay model......................................................................42

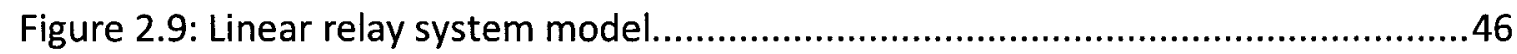

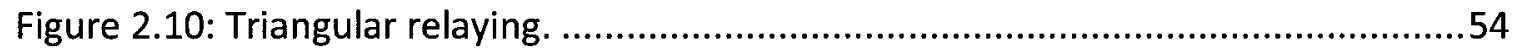

Figure 3.1: Theoretical throughput performance of startover relaying vs. actual throughput performance ( $K=1536$, BPSK).

Figure 3.2: Theoretical throughput performance of combine relaying vs. actual throughput performance ( $K=1536$, BPSK, $\gamma_{S D}=-6 \mathrm{~dB}$ ).

Figure 3.3: Theoretical throughput performance of combine relaying vs. actual throughput performance ( $K=1536, B P S K, \gamma_{S D}=-2 \mathrm{~dB}$ ).

Figure 3.4: Theoretical throughput performance of combine relaying vs. actual throughput performance ( $K=1536, \mathrm{BPSK}, \mathrm{\gamma}_{\mathrm{SD}}=+2 \mathrm{~dB}$ ).

Figure 3.5: Theoretical throughput performance of continue relaying vs. actual throughput performance ( $K=1536$, BPSK). .65

Figure 3.6: Comparison of linear relay performance: $K=1536, B P S K, \gamma_{S D}=-6 \mathrm{~dB}$........67

Figure 3.7: Comparison of linear relay performance: $K=1536, B P S K, Y_{S D}=-4 \mathrm{~dB}$........69

Figure 3.8: Comparison of linear relay performance: $K=1536, B P S K, \gamma_{S D}=-2 \mathrm{~dB}$.......69

Figure 3.9: Comparison of linear relay performance: $K=1536, B P S K, \gamma_{S D}=0 \mathrm{~dB}$.........70

Figure 3.10: Comparison of linear relay performance: $K=1536, B P S K, \gamma_{S D}=+2 \mathrm{~dB}$.....70

Figure 3.11: Comparison of linear relay performance: $K=1536, B P S K, \gamma_{S D}=+4 d B$.....71

Figure 3.12: Theoretical throughput performance of startover relaying vs. actual throughput performance ( $K=384$, BPSK).

Figure 3.13: Theoretical throughput performance of combine relaying vs. actual throughput performance $\left(K=384, B P S K, Y_{S D}=-6 \mathrm{~dB}\right.$ ). 
Figure 3.14: Theoretical throughput performance of combine relaying vs. actual throughput performance ( $K=384$, BPSK, $\gamma_{S D}=-2 \mathrm{~dB}$ ).

Figure 3.15: Theoretical throughput performance of combine relaying vs. actual throughput performance ( $K=384$, BPSK, $\gamma_{S D}=+2 \mathrm{~dB}$ )

Figure 3.16: Theoretical throughput performance of continue relaying vs. actual throughput performance ( $K=384$, BPSK). 75

Figure 3.17: Comparison of linear relay performance: $K=384, B P S K, \gamma_{S D}=-6 \mathrm{~dB}$........76

Figure 3.18: Comparison of linear relay performance: $K=384, B P S K, \gamma_{S D}=-4 \mathrm{~dB}$.........76

Figure 3.19: Comparison of linear relay performance: $K=384, B P S K, \gamma_{S D}==-2 \mathrm{~dB}$.......77

Figure 3.20: Comparison of linear relay performance: $K=384, B P S K, \gamma_{S D}=0 \mathrm{~dB} \ldots \ldots \ldots .77$

Figure 3.21: Comparison of linear relay performance: $K=384$, $B P S K, Y_{S D}=+2 \mathrm{~dB}$.........78

Figure 3.22: Comparison of linear relay performance: $K=384, B P S K, \gamma_{S D}=+4 \mathrm{~dB}$........78

Figure 3.23: Comparison of linear relay performance: $K=384, B P S K, \gamma_{S D}=+6 \mathrm{~dB}$.......79

Figure 3.24: Theoretical throughput performance of startover relaying vs. actual throughput performance ( $\mathrm{K}=384,16-\mathrm{QAM})$.

Figure 3.25: Theoretical throughput performance of continue relaying vs. actual throughput performance ( $K=384,16-Q A M)$. .81

Figure 3.26: Comparison of linear relay performance: $K=384,16-\mathrm{QAM}, \gamma_{S D}=-2 \mathrm{~dB} \ldots \ldots 82$

Figure 3.27: Comparison of linear relay performance: $K=384,16-\mathrm{QAM}, \gamma_{\mathrm{SD}}=0 \mathrm{~dB} \ldots \ldots .84$

Figure 3.28: Comparison of linear relay performance: $K=384,16-\mathrm{QAM}, \gamma_{S D}=+2 \mathrm{~dB}$....84

Figure 3.29: Comparison of linear relay performance: $K=384,16-\mathrm{QAM}, \gamma_{S D}=+5 \mathrm{~dB} \ldots . .85$

Figure 3.30: Comparison of linear relay performance: $K=384,16-Q A M, \gamma_{S D}=+10 \mathrm{~dB} . .85$

Figure 3.31: Comparison of linear relay performance: $K=384,16-Q A M, \gamma_{S D}=+15 \mathrm{~dB} . .86$

Figure 3.32: Optimum relaying vs. single-hop performance (BPSK). .87

Figure 3.33: Optimum relaying vs. single-hop performance (16-QAM) .......................8 8

Figure 4.1: Cell sectors....................................................................................90

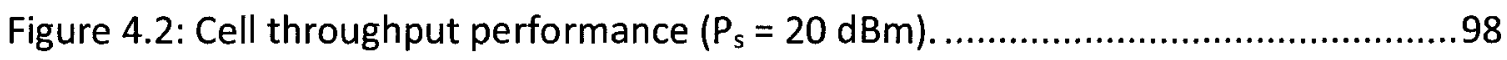

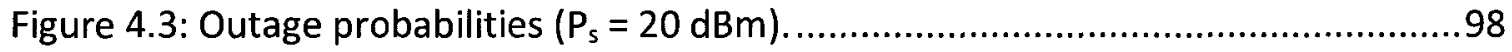

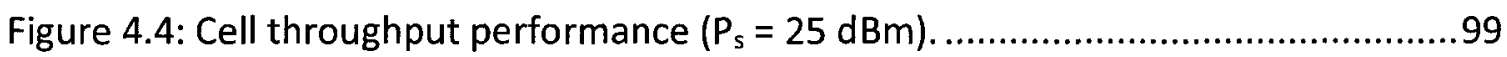

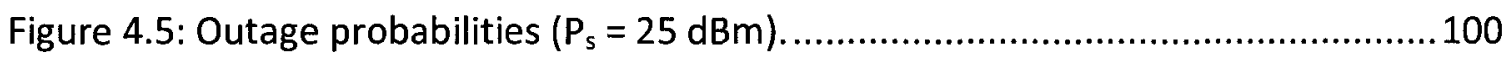

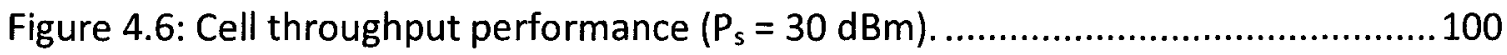

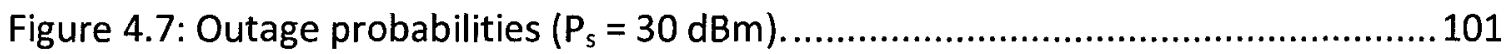

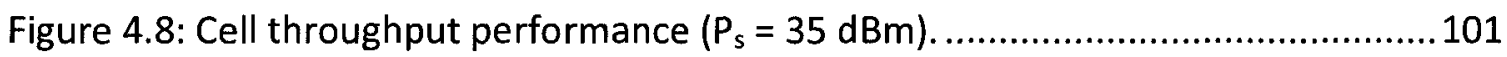

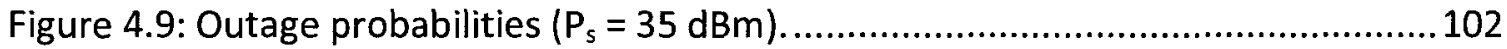

Figure 4.10: Maximum cell throughput profile................................................... 103

Figure 4.11: Minimum cell outage profile. ...................................................... 103

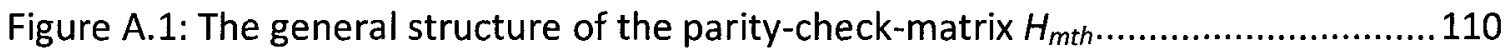




\section{Introduction}

There have been many significant advances in wireless communication over the last three decades. These enhancements have brought hypothetical communication services to life, including voice cellular communications, data cellular communications, wireless computer networks and personal area networks. Sophisticated communication procedures and algorithms found their way into real applications because of the advancements in integrated circuits, radio frequency electronics and signal processing.

Wireless communication has been the target of extensive research for a long time. The research field has been inspired by the many challenges encountered in wireless environments. These challenges include resource limitations, mobility, channel impairments, and the variable number of end users. The different aspects of wireless systems were targeted by different research communities. The data and computer network communities are concerned with the development of standard protocols for data flow control, network routing, link layer error control, and media access control. The communication theory, information theory and signal processing theory communities are more concerned with the application layer (through source coding) and the physical layer (through channel coding, signal representation, channel impairments mitigation, etc.).

Based on an information theory point of view, wireless network channels are classified into three broad types: multiple access channels, broadcast channels, and relay channels [1]. The multiple access channels are used by multiple transmission 
sources transmitting to a single destination. The broadcast channel is the converse; one transmission source transmits to different destinations simultaneously. On the other hand, the relay channels consist of one source, one destination and a number of extra channels between the source and the destination to facilitate the end-to-end transmission.

Despite the aforementioned channel classifications, most wireless applications nowadays use a single-hop transmission. Message signals travel over a single-hop from the source transmitter towards the destination receiver. While on their way to the destination, signals experience variable channel distortions such as path loss, shadowing, fading, interference and others. Although many of these have been partially or fully overcome through different strategies like error control (channel coding and flow control), equalization, diversity, interference cancelation, channel reuse and others, some problems remain.

\subsection{Problems with Single-Hop Transmission}

Single-hop transmission involves a pair of wireless terminals communicating with each other directly; they could be close together or far apart. Although single-hop transmission (between physically wired base stations and wireless end-terminals) has been widely used and implemented in both cellular and data networks, it faces a number of problems; the single-hop transmission is incapable of fulfilling the future demand on wireless networking [2][3]. 
The main concerns in the future (4G) wireless networks are ubiquitous coverage, maximizing cell capacities, achieving high data rate (up to $1 \mathrm{~Gb} / \mathrm{s}$ for stationary/nomadic users and $100 \mathrm{Mb} / \mathrm{s}$ aggregate cell rates for mobile users in large areas), minimizing time delay, minimizing error rate, and reducing the cost of the resources used. Single-hop transmission in cellular networks provides limited coverage if end users are far away or if the line of sight (LOS) is not present between the base station and the wireless terminal. These two cases, while operating at higher frequency spectrums $(2 \mathrm{GHz}$ and above), make the transmitted signals more vulnerable to channel impairments, i.e. path loss, fading, shadowing, and interference [2][3][4].

The second problem with single-hop transmission is the limitation on achieving high data rates. This problem is mainly due to the limitation on wireless resources (transmitted power, frequency channel, time channel and space) and more importantly the transmission power level. The obstacle is that at a fixed transmission power level, increasing the data rate decreases the bit signal-to-noise ratio $\left(\frac{E_{b}}{N_{0}}\right)$ linearly, which in turn increases the bit error rate and hence limits the realization of a high data-rate [2][3]. For cases when power is not limited, increasing transmission power levels introduces more interference to neighboring areas operating in the same frequency band which in turn decreases the signal-to-interference-and-noise ratio (SINR) and consequently limits the data rate [3].

The third problem with single-hop transmission is the inefficient use of wireless resources. When the single-hop channel experiences high path loss or deep fading, the 
single-hop transmission becomes more costly and the trade-off is longer use of resources [3].

The solutions to overcome these obstacles are of two types: either architectural modifications of the wireless network or using advanced transmission and reception techniques. The architectural modification solutions include increasing the density of base stations/access points (BSs/APs), deployment of micro-/pico-cells, and radio-onfiber. However these solutions are not cost-effective. Advanced transmission and reception techniques including distributed antennas, smart antennas, interference cancellation, and multiple-input-multiple-output (MIMO) aren't always practical (like having distributed antennas in small wireless terminals) [2][3].

In wireless networks, reliability plays an important role in meeting quality-of-service (QoS) requirements. Reliability is measured in terms of outage probability. Outages are mainly caused by fading channels as well as shadowing. Fading and shadowing could be mitigated by diversity: time diversity, frequency diversity, or space diversity. As a matter of fact, time and frequency diversities are expensive options in congested networks. On the other hand, space diversity implies increasing the number of antennas spaced at an order of wavelengths; which is also impractical in small wireless terminals [3].

\subsection{Relaying}

A solution to the single-hop transmission problems is relaying. Relaying is one of the most promising techniques to achieve ubiquitous coverage at high data rates. When using relaying, signals reach the destinations over multiple hops. It enables effective 
distribution and collection of signals to and from wireless terminals. It also costeffectively compensates for the channel impairments, as it offers mitigation on pathloss, fading and shadowing, especially for those terminals far away from BSs. Hopefully, high data coverage then can be effectively extended and made ubiquitous as promised at a low cost [2][3].

The main reason behind the cost-efficiency of relays is that relays do not require wired backhaul connectivity with BSs or APs. Actually, the direct connection to the backbone is what makes relays different from APs and BSs. Also, the power transmitted by relays is expected to be lower than the one transmitted by BSs, since the area covered by a relay is less. Furthermore, relays require lower-cost towers and less maintenance than BSs [2][3].

Another feature of relaying is diversity, because relays act like virtual antennas. This feature makes relaying effective against shadowing and fading impairments [4]. So, signals would reach the destination with better quality (high diversity) improving the decision at the receivers. Also, different advanced modulation and coding techniques can be incorporated with relaying, to enhance the overall performance [2].

Relaying also improves the outage capacity to achieve better reliability as it serves as multihop-diversity in diversity-limited fading environments. There exists an optimal number of hops that optimizes the end-to-end outage probability. This number increases with increasing the data rate [4]. Another good feature of relaying is that it can be used to divert traffic from congested areas to other areas with lower traffic load [2]. 
The relay network can be either infrastructure-based or mobile (ad hoc). In infrastructure-based relay networks, the relays are positioned in fixed strategic locations, while in ad hoc networks, the relays are mobile [2][3].

There are four protocols of relaying: amplify-and-forward (AF), decode-and-forward (DF), compress-and-forward (CF), and compute-and-forward. In AF, relays amplify signals and retransmit them, whereas the DF relays decode source messages, re-encode them, and retransmit them. On more advanced relaying protocols, the CF relays forward quantized and compressed versions of the received signals. The latest relaying protocol, compute-and-forward relaying, enables relays to decode linear equations of the transmitted messages using noisy linear combinations provided by the channel, which the destination can solve for its desired messages [5][6].

Full-duplex (FD) relaying allows the relay to receive and transmit simultaneously over the same channel. In fact, this is not practical. In reality, relays incorporate time division duplex (TDD) or frequency division duplex (FDD) to be able to receive and transmit over two different channels. TDD/FDD relaying can take place as simultaneous relaying or non-simultaneous relaying. Simultaneous relaying allows the relay and source to transmit simultaneously, whereas non-simultaneous relaying allows only one transmitter at the same time, either the source or the relay [7][8][9].

\subsubsection{Research on Relaying}

Relay channels were first introduced by van der Meulen [10]. Cover and El Gamal derived the upper and lower bounds for the performance of relay channels using the cut-set theorem (or max-flow min-cut) [11]. The cut-set theorem states that the 
maximum achievable rate is upper bounded by the minimum cut. The cut is obtained by separating the network into two parts, and evaluating the mutual information with respect to this cut [12]. The coding schemes used to derive the lower bound were Markov superposition encoding, random partitioning, and successive decoding [11]. The codebooks used belong to different sizes [11]. After that, a lot of researches have tackled relay channels using different models.

The ergodic and outage capacities for Rayleigh relay channels were studied in [9] for many cases of interests. It was shown that relaying gives better gains for both ergodic and outage capacities over direct transmissions. More importantly, power allocation was found to increase the relaying gain even for high signal-to-noise-ratios (SNRs) [9]. In [13], Kramer, Gastpar and Gupta studied relay channels for both pure additive white Gaussian noise (AWGN) channels and phase fading channels. The coding scheme used was regular block Markov encoding and windowed decoding. It was shown that DF relaying supersedes AF relaying over AWGN channels, and the relay gain is optimal for phase-fading when the relay is close to the source transmitter, provided that the channel state information (CSI) is available to the receiver only [13].

In [14], the authors found that for multiple relay networks (as for ad hoc networks) consisting of $n$ terminals while using point-to-point coding, the throughput per terminal decreases in the order of $\frac{1}{\sqrt{n}}$ for optimally placed terminals, and decreases in the order of $\frac{1}{\sqrt{n \log _{2} n}}$ for random placed terminals. Both results suggest that the throughput goes to zero as the number of terminals goes very high [14]. The traffic model used was that all nodes in the network are split into sources and destinations at random. 
Another model considering multiple-relay networks was done by Gastpar and Vetterli in

[12]. They used network coding in a cooperative relay model (in contrast to multi hop) to show that the $n$-node network capacity per terminal behaves like $\log _{2} n$ as the number of nodes goes very high under the assumption that only a single active link is present at a time and other nodes act as relays.

In [7], Nicholas Laneman did a thorough analyses for synchronous (Laneman used the term "cooperative") and asynchronous (Laneman used the term "non-cooperative") transmission of relay channels using information theoretical tools. He showed, in the context of relaying, that synchronous transmission capacity supersedes asynchronous transmission capacity by a factor of $M$ for low SNRs, and by $M \mathrm{~b} / \mathrm{s} / \mathrm{Hz}$ for high SNRs when having $M$ full-duplex cooperative transmitters (each terminal plays the role of a relay for others). For example, for a one relay channel the capacity of cooperative transmission would be higher than the non-cooperative transmission capacity by a factor of 2 at low SNRs, and by $1 \mathrm{~b} / \mathrm{s} / \mathrm{Hz}$ at high SNRs. He also showed that there is a gain in the outage probability when using relaying in fading channels. The outage probability is reduced by $\frac{1}{S N R^{2}}$ for $A F$ half-duplex relaying, while for a direct transmission the reduction is in the order of $\frac{1}{\mathrm{SNR}}$. Laneman also showed that the DF half-duplex relaying outage probability behaves similar to the direct channels, due to the extra effort played by the relay to decode and re-encode messages.

Hasna and Alouini studied a multi hop relay system when the direct link between the source and the destination is deeply faded (or absent) over flat Rayleigh-fading 
channels. They found that DF outperforms AF in terms of bit error rate and outage probability at low SNRs and both behave similar at high SNRs [8].

Castura and Yongyi introduced rateless coding to relay networks. They analyzed a single-relay system based on the framework of MIMO systems, and found the achievable rate region for such a system. The relay model they presented was TDD relaying with simultaneous transmission (source and relay transmit simultaneously). The code they used was the Raptor code [15]. The relay aids the transmission process by simultaneously transmitting the same code bits the source transmits. The destination combines both transmitted code bits and decode. They found that the maximum singlerelay system throughput would be very close to the capacity of MIMO channel with diversity order of $2 \times 1$. The derived achievable rates resulted from solving the rate bounds of relay channels. The results were validated over a quasi-static Rayleigh fading relay channel, where the channel gains remain constant over the period of an entire codeword transmission [15].

In [16], a queued cooperative relay system using Fountain rateless coding over fading channels was examined. The relays were assumed to have buffers to allow the source to transmit more as the relays take over. Also, the relay transmission was assumed to be full duplex. Two relay selection strategies were studied, namely, relay selection diversity and relay cooperation with strong inter-relay links. The relay selection diversity selects the relay having the best link with the source to forward messages to the destination. The second selection strategy dictates that all relays cooperate to forward the message to the destination. It was assumed that the direct channel link between the source and 
the destination is absent, resembling a multi-hop cascade relaying. The relays transmit at orthogonal channels using different sequence spectrum spreading. The first strategy provides both load balancing and diversity to the transmission system, but suffers from a smaller stability region. On the other hand, the relay cooperation strategy outperforms any other strategy by providing diversity, load balancing, a wider stability region, and enhances the system throughput [16].

A new relay combining strategy was introduced in [17], using rateless coding. The new combining strategy, mixed combining $(M C)$, is a mixture of the two traditional combining strategies, namely energy combining (EC) and information combining (IC). The EC is based on combining repeated code bits using maximal-ratio as the relay retransmits the source transmissions. In the IC, the relay transmits different code bits and the destination combine the two transmissions by using a composite generator matrix governing the two streams. The proposed $M C$ strategy was derived from these two strategies. The model used was based on TDD, simultaneous relaying over orthogonal channels. It was found that IC outperforms EC, and the proposed $M C$ outperforms both strategies. However, $M C$ is subject to optimization to perform as required [17].

In [18], a Fountain code (Raptor code) was examined over slow deeply-interleaved fading relay channel. The study extends the work of [15] to three rateless cooperative protocols, namely distributed space-time code (DSTC), time-division (TD), and two-hop (cascade multi-hop). The DSTC uses Alamouti space-time coding during the cooperation phase, during which the relay has already decoded the message and has started the 
transmission simultaneously with the source forming a MIMO channel. For the TD relaying, it is assumed that the relay takes over after it successfully decoded the source message (non-simultaneous relaying) and the source stops the transmission. It was found that all the three protocols achieve zero outage probability without CSI at the transmitters. Also it was found that under the same total power budget, TD protocol outperforms DSTC protocol while the two-hop performs the worst [18].

In [19], the performance of Raptor codes was tested over collaborative relay networks. Information accumulation was facilitated in this research by using Fountain codes, in contrast to the traditional energy accumulation widely used in literature. The relaying model was assumed non-simultaneous where the source stops transmission as soon as relaying takes place. Two non-simultaneous relaying strategies were analyzed, namely quasi-synchronous and asynchronuous. In the quasi-synchronous relaying strategy, there has to be a certain number of relays that have successfully decoded the source message before the relays take over. On the other hand, asynchronuous relaying dictates that as soon as the first relay decodes the source message successfully, the source will stop and the relay will take over feeding the destination and other relays which may cooperate later. Relays transmit over orthogonal channels. In both scenarios, the direct source-to-destination link is assumed absent. It was found that for the quasisynchronous relaying there exists an optimum number of active transmitting relays that minimizes the total power expenditure. The asynchronuous relaying was found efficient in reducing the latency of transmission and also saving the total transmit energy [19]. 
Rateless coding also finds its way to MIMO channels, which is the generic model of relay channels. It has been found that rateless coding enhances the diversitymultiplexing-tradeoffs and fit naturally over MIMO channels [20][21].

\subsubsection{Standards Incorporating Relaying}

Relaying has already been introduced in industry, and efforts to standardize relaying are being accepted. For cellular systems, the first effort was lead by the European Telecommunications Standards Institute/Digital Enhance Cordless Telephony (ETSI/DECT) as they specified fixed relays for both voice and data using TDD multiplexing [2]. The Wireless World Initiative New Radio (WINNER) project promises to provision ubiquitous high data rate coverage from small range to wide range in the $4^{\text {th }}$ generation (4G) of radio systems. WINNER has already adopted the concept of relay-based deployment. Also, IS-95 and Universal Mobile Telecommunications Systems (UMTS) terrestrial radio access (UTRA) FDD use FDD for code-division-multiple-access (CDMA) relaying [2].

Wireless LAN (WLAN) IEEE 802.11 (2.4 GHz and $5 \mathrm{GHz}$ frequency bands) has adopted two services, namely: basic service (BSS) and independent basic service (IBSS) also known as ad hoc. The basic service allow terminals to communicate through the access point (AP) only, while the ad hoc mode allows terminals to communicate directly without the need of APs. The ad hoc mode may be used to relay packets; however it doesn't perform well because it uses a contention-based (CSMA) media-access-control (MAC) protocol. Multihop mesh techniques soon will be available under the IEEE 802.11s ESS mesh standard [22]. 
As for metropolitan area networks (MAN), IEEE 802.16 (2-11 GHz frequency band) has already adopted relaying under the IEEE $802.16 \mathrm{~m}$. The IEEE 802.16 standard is designed for point-to-multipoint (PMP) to provide high data rate (up to $100 \mathrm{Mb} / \mathrm{s}$ for high mobility and $1 \mathrm{~Gb} / \mathrm{s}$ for low mobility) with line of sight (LOS) coverage of $5 \mathrm{~km}$. The IEEE 802.16a covers up to $50 \mathrm{~km}$, allowing non-LOS (NLOS) with two basic modes: PMP and mesh mode. The mesh mode allows multi-hopping through wireless terminals. Infrastructure-based relays will soon be adopted by the IEEE 802.16 to extend PMP coverage to NLOS wireless terminal [4][22][23].

Low-rate wireless personal area network (WPAN) IEEE802.15.5 and Zigbee $(2.4 \mathrm{GHz}$ and $868 / 915 \mathrm{MHz}$ frequency bands) will soon provide an architectural framework standard adopting multi-hopping for WPAN devices [22]. Sensor networks will benefit a lot from the new standards.

ETSI-broadband radio access network (BRAN), and ETSI-high-performance LAN (HiperLAN/2) already have the capabilities to adopt relaying through the ad hoc mode. It is found that the multi-hoping works better with HiperLAN/2 than with other WLAN standards as it uses TDD TDMA (OFDMA)-based MAC while others use CSMA-based MAC [2].

LTE-A has adopted relaying to enhance high data rate and group mobility. The relays in LTE-A operates in two modes, namely inband and outband. The backhaul in inband relays share the same band with direct wireless end user links, while the backhaul outband relays operate at different band. LTE-A relays are classified as transparent, 
where the end user does not know about the relay, and non-transparent, where the end user knows about the relay [24].

\subsubsection{Problems with Relaying}

Now that we have seen the incentives for using relying in wireless systems, it is time to look into the challenges facing the deployment of relays. The first problem is related to the relaying duplex transmission. The capacity of relay channels is maximized when using full-duplex relaying, where the relay receives and transmits simultaneously using the same channel. However, full-duplex relaying is not practical, as it causes unmanageable problems at the circuit level in the relay station. It also causes extra interference at the destination, degrading the overall performance of the reception capacity. Instead, time-division-duplex (TDD) or frequency-division-duplex (FDD) transmission is more practical. While TDD/FDD relaying eliminates the problem of transmission and reception at the relay station, the throughput is degraded (by a factor of 2 if using half-duplex). Also, TDD/FDD requires more channel-resources to eliminate the interference at the destination caused by simultaneous transmission from the source and the relay. The extra relay-to-destination channel-resources can be allocated using TDMA or reusing neighboring-cell's frequency-TDMA channels. All these extra resource allocations have to be well designed and managed [9].

The second problem with relaying is the resource management problem. In addition to the channel allocation for relaying, mobility and handoff need to be managed in the presence of relays. The extra management tasks arise from the fact that end terminals need to associate with relays in addition to the association with BSs. Resource allocation 
and management can be either central or distributed. In the central resource allocation, the AP or the BS distributes the resources to the relay links and the end terminal links. To be able to do so, the BS needs to have global CSI and use efficient centralized schedule and routing algorithms. This would create substantial overhead for the feedback, and the processing will increase accordingly. For the distributed resourceallocation, relays and terminals need to have higher processing capabilities in order to contribute. Also, extra tasks will be assigned to the relays and the end terminals to do scheduling, and interference management. A better prospective solution combines the two resource management methods, where BSs, relays and maybe end-terminals contribute in the resource allocation and management process [2].

In addition to all these problems, different real life impacts on the relay performance haven't been studied well. For example, the impacts of delay, throughput, coverage, and reliability on relay systems have not been thoroughly studied [3].

As relays introduce extra transmissions, another serious problem needs to be tackled, which is the power consumption. Power consumption is expected to increase and the impact might be serious. Few studies have tackled this issue with limited results on the capacity [2][3][9].

It was found in the literature, codes that were used in the context of relaying are of three types: peer-to-peer, network coding, and space-time coding. Although most of the studies have assumed certain coding schemes, they mostly were general and did not give details on what code will give good relay performance. 


\subsection{Type II HARQ}

As an effort to improve the performance of HARQ type I, type II HARQ was introduced. It dictates that packets with uncorrectable errors should be utilized instead of being discarded. In principle, there are two approaches to utilize packets with uncorrectable errors, namely code combining and incremental redundancy (IR).

\subsubsection{Code Combining}

In code combining (Chase combining) type II HARQ, a packet consisting of information and CRC bits of code rate $\mathrm{R}$ is transmitted through the channel and when received with uncorrectable errors, the receiver asks for a re-transmission. So, the transmitter resends the exact packet again. The receiver stores the received $L_{\text {tran }}$ packet(s) and combines them to improve the decoder's decision. The decoder uses soft decision (maximum-likelihood) rather than hard decision. Each received packet is given a reliability weight as per the channel estimation associated with that packet. Channel can be estimated using training bits to give a reliability measure and estimation of the

channel SNR. The final code rate is $\frac{\mathrm{R}}{\mathrm{L}_{\mathrm{tran}}}$ and the resulting minimum distance is $\mathrm{L}_{\text {tran }}$ times the basic code's minimum distance [25].

The maximum-likelihood decoder (code combiner) chooses the packet that maximizes the conditional probability between the received sequences and the repeated packets. Every repeated packet is tagged by a reliability weight before combining it with other repetitions. The decoder finds the sequences that result in the least bit-disagreements between the repeated packets [25]. 
The reliability weight for channels tags the less reliable packets with less weight, and vice versa. In the BSC channel, it is the natural logarithm of the ratio of success probability to failure probability. As so, packets with high errors would be given less weight while packets with fewer errors would be weighted more. For the AWGN, it is the reciprocal of the channel noise variance. For higher noise, the variance would be more and the weight would be less and vice versa [25].

Chase asserted that code combining would allow communication as long as the channel capacity is finite and not limited by the code used (error rate must not be greater than $12 \%$ for maximum-likelihood Viterbi decoder and not greater than $25 \%$ for algebraic decoders). He emphasized on the fact that code combining would not necessarily improve the code gain versus SNR as for AWGN channel, however the real advantage of code combining is being adaptive to jamming threats where the conventional diversity combining breaks down. Conventional diversity simply combines symbols over different time slots. Jamming means long and strong interference or fading that may result in loss of communication. Chases showed the bit error performance of using code combining was enhanced; however the throughput gain versus SNR was not really improved [25].

Hard decision can also be used in code combining. In [26], instead of using soft decisions, the packets are hard decided. Then, repeated packets are XOR'ed to locate erroneous bits. These erroneous bits are brute-forcedly inverted until the CRC check indicates correctness. This lengthy search would be suitable for small data packets as the computation is expected to grow exponentially with the packet size. 


\subsubsection{IR Type II HARQ: Rateless Coding}

IR Type II HARQ is basically based on sending out extra bits rather than resending the whole packet as in code combining. After the first packet is sent with CRC bits, the decoder tries to decode the data packet. However, if the decoding is not successful then a NACK is sent back to the transmitter requesting for more parity bits. The transmitter keeps sending more parity checks until receiving ACK message or transmission is timed out.

IR type II HARQ requires a code that is attributed with hypothetically endless parity bits. These codes are widely named "rateless codes". Rateless coding employs error correcting codes that fall in one of two broad categories, namely fountain codes and rate compatible (RC) codes [27][28][29][30]. Raptor codes and Luby Transfer (LT) codes are the two famous fountain codes. The fountain codes basically generate code bits pseudo-randomly on the fly. The receiver can decode the transmitted message by collecting enough code bits.

The LT code was invented earlier by Luby in 1998 originally for Binary Erasure Channels (BEC). LT codes can generate limitless source packets while the decoder picks and decodes received packets until successfully recovering the original information packet. LT encoding process works as follows: [28]

1) A degree is chosen randomly for the encoding symbol from a degree distribution

2) A uniformly random $i$ distinct source symbols are chosen as neighbors of the encoding symbol.

3) The value of the encoding symbol is the exclusive-or of the $i$ neighbors. 
The degree distribution is carefully designed to yield good results. The decoder needs to know the degree and set of neighbors of each encoding symbol. This information can be sent in the overhead of the transmitted packets or prior agreed upon through pseudorandom procedure that both encoder and decoder know about [28].

LT decoder uses the Belief Propagation (BP) algorithm to decode received codewords. The complexity of encoding and decoding of LT code grows as $O(\mathrm{k} \ln (\mathrm{k}))$, where $\mathrm{k}$ is the number of input symbols which is a drawback when packet size is large.

Raptor codes are another type of Fountain codes that were invented by Shokrollahi to overcome the drawbacks of LT codes complexity. Raptor codes encoding and decoding complexity grows as $\mathrm{O}(\mathrm{k})$, which is linear. Basically, a Raptor code is a concatenation of an outer linear code (e.g. LDPC) with LT as an inner code. Though showing high throughput performances at lower SNRs, Raptor code throughput at high SNR is limited and not reaching near-Shannon limit region [29].

RC codes, on the other hand, are derived from fixed-rate mother codes, e.g. convolutional codes and low-density-parity-check (LDPC) codes. The RC code is generated by puncturing/combining the mother code to generate shorter codewords, and by extending the mother code to generate longer codewords. The rate compatibility dictates that shorter codewords are subsets of longer codewords. The shorter codewords are sent first to the receiver. If the decoding process fails at the receiver, more code bits are sent to complete the next higher rated codeword. This process goes on until the receiver decodes successfully [27][30]. 
One approach of designing a RC code is to pick a low rate code and start building a family of higher rated codes out of it, using puncturing. Another approach is to generate a family of different rated codes from the mother code $t$ : higher and lower rated codes. In both approaches, the higher rated codes have to be always a subclass of the lower rated codes [31]. A good practice in designing $\mathrm{RC}$ codes is to have a fixed pair of encoder and decoder to encode and decode all different subsets of that code [31].

The transmitter starts with the highest rated code packets and moves on to the lowest rated code packet, adapting to the channel conditions without much feedback from the receiver. The basic feedback required is ACK/NACK messages. The gain is not only improving the error control performance but also improving the total throughput, given that each code of the RC code family has a good performance.

Most of researches on IR type II HARQ were based on the convolutional codes, turbo codes, LDPC codes, and Fountain codes. In [31], Hagenuer developed an RC code based on convolutional code using a computer search. He constructed a family of RC using puncturing of a $1 / \mathrm{N}$ convolutional code. The puncturing rule he applied was: all punctured bits in the lower rated code have to be also punctured in the higher rated codes. He constructed families of RCPC codes through an iterative procedure. First, a mother convolutional code is chosen of certain rate $1 / \mathrm{N}$, memory $\mathrm{F}$, generator $\mathrm{g}$, and puncturing period $\mathrm{P}$. Then, different $\mathrm{RC}$ puncturing schemes are applied to the code to achieve different higher rates. The free distance of each resulted higher rate is evaluated and stored. These steps are repeated for all possible RC puncturing patterns. Finally, the best code rate with highest free distance is chosen. The free distance is an 
indication of how good the code is. It was noticed that increasing the period of puncturing yields better codes with greater free distances and therefore better bit error rate performances [31].

Basically, Hagenuer's proposal was to send the mostly punctured packet at first. Then, if decoding wasn't successful the transmitter will send a selected number of punctured bits at a time until successfully decoding the packet without any error. Analysis and simulation over Gaussian channel and Rayleigh fading channel using a $1 / 3$ convolutional code as a mother code showed very good results in the sense of throughput and frame error rate (FER). The disadvantages were mainly the complexity of decoding and memory utilization to store the packets [31].

[32] presents a different interesting approach using $1 / 2$ convolutional codes in type II HARQ. First, a high rate punctured convolutional code derived from $1 / 2$ convolutional code is used. Then, lower RC codes are derived from that code, by just turning zeros in the puncturing matrix into ones until reaching the mother code rate of $1 / 2$. The next step is to extend the mother code to even lower rate codes. The extension is a straightforward operation; part of the packet bits is retransmitted again. The extension operation was summarized by a repetition matrix which is similar in concept to the puncturing matrix, but instead of having 0's and 1's, the elements are of 1 values or more. The paper disserted that all codes derived from a noncatastrophic high rate code are also noncatastrophic. The results were showed an outperforming throughput against code combining and also channel adaptive capability to changing channel states [32]. 
Other high-performance codes such as turbo codes and LDPC codes made it possible for IR type II HARQ to achieve near-Shannon-limit performance[33][34][35][36]. As in convolutional codes, turbo codes and LDPC codes are made rate compatible through puncturing and extending their mother codes. The drawback accompanied with puncturing and extending is the computational complexity [37]. Although complexity may be enhanced, it is at the cost of lowering the throughput performance [37]. In [35], Mohammedreza et al. could reach about $0.7 \mathrm{db}$ from Shannon limit. It was obvious that type II HARQ's performance is based mainly on the mother code used and the puncturing/extending approach used.

\subsubsection{RC/QC-LDPC Codes}

Structured and random LDPC codes perform very well over different channels. They are able to perform very close to the Shannon limit [38][39]. LDPC codes are decoded using belief propagation algorithms. Since LDPC codes are sparse codes, the decoding complexity grows linearly with the length of the code [40][41]. However, the encoding can be complex, especially with random LDPC codes.

The complexity of encoding can be simplified by using well-structured LDPC codes. Among other structured LDPC codes, quasi-cyclic LDPC (QC-LDPC) codes show low complexity for both encoding and decoding. The parity-check-matrix of a QC-LDPC code is an array of circulant matrices (also called circulants). The encoder of the QC-LDPC codes can be realized by using either serial or parallel shift registers. The complexity of encoding QC-LDPC codes is linear. The decoding is less complex than non-QC-LDPC codes due to the modular structure of the QC-LDPC codes [40][42][43][44]. 


\subsection{Thesis Objective}

This research work aims for a better relay communication system model. The main objective is to develop a relay system model that operates on its maximum throughout with low negotiation message overhead and low resource allocation management. The message overhead originates from the fact that the transmission components (source, relay and destination) need to synchronize in a way to decide when to relay and what resources are needed for relaying. The resources involved in the relaying process are power and channel allocation.

The second objective is to explore the suitability of rateless coding, and more specifically RC/QC-LDPC codes, when used with relaying.

The third objective is to develop an adequate strategy of relaying, and how the destination can make the most use of that strategy.

The fourth objective is to develop a theoretical performance model for the system at hand.

\section{Thesis Statement}

This thesis work develops and evaluates a relay system that uses rateless coding and non-simultaneous TDD relaying. It develops the rateless coding by using IR type II HARQ based on RC/QC-LDPC code. The IR type II HARQ used here is modified in a way to suit relaying systems and to have the least negotiation overhead. This thesis also finds the best relaying and reception approach to maximize the throughput of the system as a 
whole. Theoretical models are derived that well describe the relay system using IR type II HARQ.

\subsection{Research Contributions}

This research further studies IR type II HARQ application to wireless relay networks based on RC/QC-LDPC codes. Castura and Yongyi in [15] did similar study based on Raptor codes and simultaneous relaying. Here, the study is based on RC/QC-LDPC code with non simultaneous relaying. The theoretical performance derived here is found consistent with the one that was done in [15], but the approach here is different and more tractable.

A significant result of this research finds that relaying new code bits (continue relaying) is better than relaying repeated code bits, in the context of wireless relay networks.

Another contribution of this research is the introduction of a new relay association mechanism that is easy to implement and yield a good performance. This approach is based on continue relaying and requires little information overhead and the decision processing is straightforward.

Also, this research shows that 3GPP2 QC-LDPC code can be modified into RC code with powerful throughput performance over relay channels.

\subsection{Thesis Outline}

This thesis presents the research as follows. Chapter 2 presents the model of "rateless coding for wireless relay networks". It starts with describing the single-hop 
system model, detailing error control methods (rate compatible coding, and type II HARQ), signal mapping, and channel model. Next, it presents the single-hop throughput performance using the aforementioned techniques. The general relay system model together with the relaying strategies are explained afterwards, based on the rateless coding and type II HARQ. The "linear relay model" is then presented, in order to be used as a test model to evaluate the developed methods of relaying. Next section presents the derivation for the theoretical performance model of the rateless coded linear relay system. The chapter concludes by generalizing the findings of the linear relaying to a more general one, which is "triangular relaying". The first part of Chapter 3, presents the simulation results of the system model, validating the theoretical performance with the simulation results. Chapter 4 applies the rateless coded relaying method to a single-cell wireless system with three relays, to evaluate the enhancements of this relaying method by presenting simulation results. Chapter 5 concludes the thesis work and lists potential future research topics. 


\section{System Model}

This chapter presents the system model of "rateless coding for wireless relay networks". The relay model considered here is the simplest model: a one-relay model, which consists of a source transmitter, a relay, and a destination receiver. Section 2.1 describes the single-hop system model, to facilitate the understanding of wireless transmission over single-hops. It explains details about CRC coding, rate compatible coding, signal mapping, channel model, decoding and type II HARQ. In Section 2.2, the throughput performance of single-hop transmission over the AWGN channel is presented. Section 2.3 presents the general one relay model, detailing type II HARQ acknowledgement, the relay association process, system operation and relaying strategies. Section 2.4 applies the relaying strategies to the framework of a linear relay model. The theoretical performance of the linear relaying is derived in Section 2.5 for the three categories of relaying modes: startover, combine and continue relaying. Section 2.6 generalizes the findings of linear relaying to triangular relaying systems for the three relaying methods.

\subsection{Single-Hop System Model}

The system model for single-hop transmission (or any individual hop in a multihop system) involves a transmitter, a discrete-time channel, and a receiver. The transmitter incorporates cyclic redundancy check (CRC) encoding, rate compatible coding, and signal mapping, and the receiver incorporates decoding and CRC checking. These components are described in the following. 


\subsubsection{CRC Padding}

Information data blocks are first padded with a CRC code, resulting in a $K$ bit message word $\boldsymbol{u}$. The CRC is used to detect transmission failures at the receiver. Although in practice CRC codes introduce a finite non-zero overhead in the message length (and therefore contribute to a slight reduction in the transmission rate since extra time is needed to transmit the CRC parity bits), and are not always able to detect transmission failures, in this work we assume the CRC introduces no overhead and flawlessly detects transmission failures. System performance analysis under more realistic assumptions is deferred as a topic of future research.

\subsubsection{Rate Compatible Coding}

The CRC padded information message, $\boldsymbol{u}$, is encoded with a rate compatible (RC) code, giving codeword $c$. An RC code has the ability to generate a set of codewords, where every higher rated codeword is a subset of the next lower rated codeword. The length difference between the consecutive codewords can be constant or variable. The design of the RC code starts with designing a basis code, called a "mother code" and its parity-check matrix is called the "mother parity-check matrix". The mother code should hold good performance criteria in terms of encoding, decoding, and the ability to be converted into a family of RC codes. It is necessary that the mother code and its RC codes perform well over the targeted channels.

To save the effort of designing a new code, this paper adopts a ready-to-use code of a well known standard: the QC-LDPC codes found in 3GPP2 (3rd Generation Partnership 
Project2) [45]. The resulting RC/QC-LDPC code is able to generate a series of paritycheck matrices $\left\{\boldsymbol{H}_{i}\right\}$ having a range of code rates from $R_{c}=0.1$ to 0.96 . The RC/QCLDPC encoder encodes the message to the lowest rate $R_{\min }=0.1$ to produce a codeword $c$ of size $10 K$.

The detailed RC/QC-LDPC code used here is explained in Appendix A.

\subsubsection{Signal Mapping}

In signal mapping, code bits are converted into symbols using one of the common modulation techniques. The bits of the codeword $\boldsymbol{c}$ are mapped into points in an $M$-ary signal constellation, where $M=2^{n_{d}}$ is the number of points in the constellation. Each symbol represents $n_{d}$ bits of the codeword $c=\left[c_{1} c_{2} \ldots c_{N_{\max }}\right]$. Assuming the code bits of the $i^{\text {th }}$ symbol are $\boldsymbol{b}_{i}=\left[\boldsymbol{b}_{i}^{(1)} \boldsymbol{b}_{i}^{(2)} \ldots \boldsymbol{b}_{i}^{\left(n_{d}\right)}\right]$, and then the transmitted symbol $s_{i}$ is

$$
s_{i}=\operatorname{SM}\left[\boldsymbol{b}_{\boldsymbol{i}}\right]
$$

where SM[.] represents the mapping between bit sequences and points in the signal constellation. In this research work two modulation schemes are used, namely BPSK and 16-QAM. BPSK has $n_{d}=1 \quad(M=2)$, while 16-QAM has $n_{d}=4 \quad(M=16)$. The constellations of both schemes are shown in Figure 2.1.

The average transmitted energy per symbol of either constellation is

$$
E_{\text {avg }}=\frac{1}{M} \sum_{m=0}^{M-1}|\mathrm{SM}[m]|^{2} \text {. }
$$

For BPSK the average transmitted energy is $A^{2}$, and for the 16-QAM the average transmitted energy is $10 A^{2}$. 


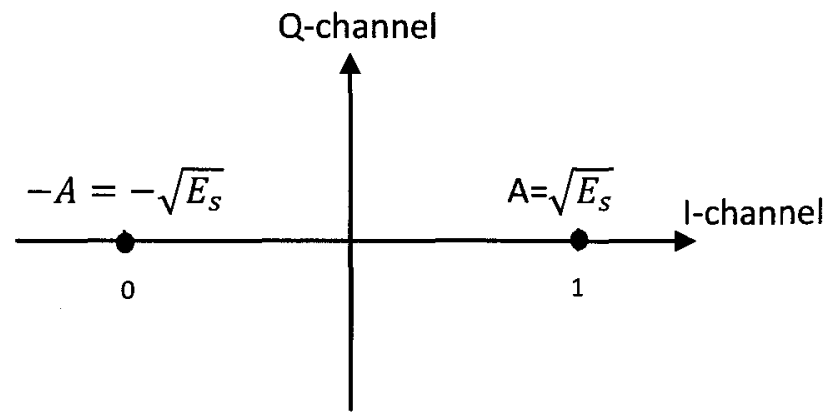

(a) BPSK constellation

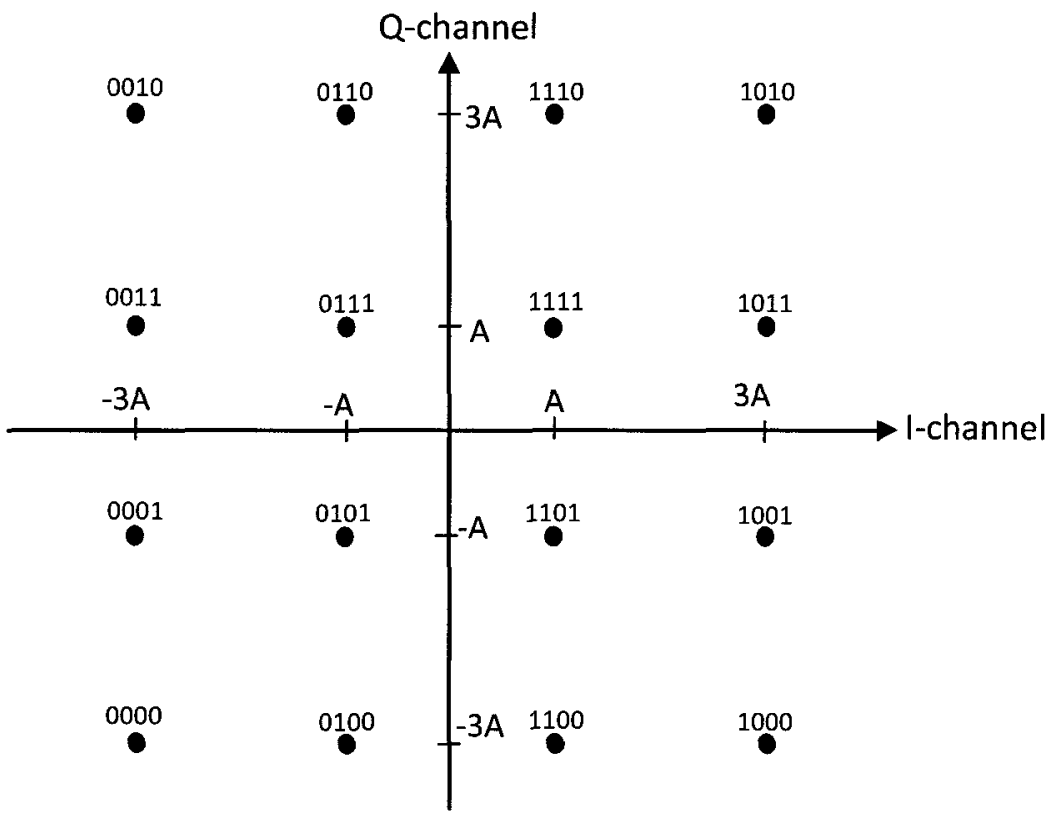

(b) 16-QAM constellation

Figure 2.1: Signal constellation for (a) BPSK and (b) 16-QAM.

\subsubsection{Channel Model}

The channel used in this research is the attenuated AWGN channel. As the transmitter transmits symbol $s_{i}$ over the channel, the received sample at time $i$ is

$$
r_{i}=\alpha s_{i}+w_{i}
$$


where $w_{i}$ is complex white Gaussian noise with zero mean and variance $\mathrm{E}\left[\left|w_{i}\right|^{2}\right]=N_{0}$. The term $\alpha$ is the attenuation factor, and it is assumed to be real. This captures the path loss encountered by the transmitted signal. In general, the received SNR $(\gamma)$ is

$$
\gamma=\alpha^{2} \frac{E_{s}}{N_{0}} .
$$

The channel model here ignores shadowing, delay spread, multipath fading and Doppler effects; it only focuses on path loss and additive white Gaussian noise.

\subsubsection{Decoding}

The decoder is based on a message-passing algorithm. We will use the method that was used by Neal [46], where likelihood ratios (LRs) are propagated instead of logarithm likelihood ratios (LLRs). The likelihood ratio $\left(\mathrm{LR}_{n}=p_{n}\right)$ of the $n^{\text {th }}$ bit in codeword $\boldsymbol{c}=\left\{c_{1}, c_{2}, \ldots c_{N_{i}}\right\}$ after observing the corresponding received sequence $\boldsymbol{r}=$ $\left\{r_{1}, r_{2}, \ldots r_{N_{i}}\right\}$, is given as

$$
\mathrm{LR}_{n}=p_{n}=\frac{\operatorname{Pr}\left\{c_{n}=1 \mid \boldsymbol{r}\right\}}{\operatorname{Pr}\left\{c_{n}=0 \mid r\right\}}=\frac{f\left(r_{n} \mid c_{n}=1\right)}{f\left(r_{n} \mid c_{n}=0\right)} \frac{\operatorname{Pr}\left\{c_{n}=1 \mid\left\{r_{i \neq n}\right\}\right\}}{\operatorname{Pr}\left\{c_{n}=0 \mid\left\{r_{i \neq n}\right\}\right\}}
$$

provided that code bits are independent and $c_{n}$ is equally probable to be zero or one.

The term $\mathrm{LR}_{n}^{\text {init }}=\frac{f\left(r_{n} \mid c_{n}=1\right)}{f\left(r_{n} \mid c_{n}=0\right)}$ is the initial value of the likelihood ratio of bit $n$, and it represents the intrinsic information about $c_{n}$. On the other hand, the term $\frac{\operatorname{Pr}\left\{c_{n}=1 \mid\left\{r_{i \neq n}\right\}\right\}}{\operatorname{Pr}\left\{c_{n}=0 \mid\left\{r_{i \neq n}\right\}\right\}}$ is the extrinsic information about $c_{n}$ which represents the contribution of other code bits.

The value $\mathrm{LR}_{n}^{\text {init }}$ is directly found from the received signal sequences provided the receiver knows the channel parameters. The intrinsic information depends on the 
channel and the type of modulation. For BPSK transmission over an AWGN channel with a single-sided PSD of $N_{0}$ and attenuation $\alpha$, the initial likelihood ratio is

$$
\mathrm{LR}_{n}^{\text {init }}=\frac{f\left(r_{n} \mid c_{n}=1\right)}{f\left(r_{n} \mid c_{n}=0\right)}=\exp \left(\frac{4 \alpha A r_{n}}{N_{0}}\right)
$$

where $\exp ($.$) is the natural exponential function. However, for 16-QAM modulation the$ situation is different. Following the 16-QAM constellation mapping mentioned earlier, the $I$-channel component is used to find the LRs of the first two bits, and the $Q$-channel component is used to find the LRs of the remaining two bits. If the complex received sample $r$ represents bits $\left[b_{1} b_{2} b_{3} b_{4}\right]$, then the LRs of bits $b_{1}$ and $b_{2}$ can be found by using $r_{I}=\operatorname{Re}\{r\}$ and the LRs of bits $b_{3}$ and $b_{4}$ can be found by using $r_{I}=\operatorname{Im}\{r\}$. In other words, the LR of $b_{1}$ is

$$
\begin{aligned}
\mathrm{LR}_{b_{1}}^{\text {init }} & =\frac{\operatorname{Pr}\left\{b_{1}=1 \mid r_{I}\right\}}{\operatorname{Pr}\left\{b_{1}=0 \mid r_{I}\right\}}=\frac{\operatorname{Pr}\left\{b_{1}=1, b_{2}=0 \mid r_{I}\right\}+\operatorname{Pr}\left\{b_{1}=1, b_{2}=1 \mid r_{I}\right\}}{\operatorname{Pr}\left\{b_{1}=0, b_{2}=0 \mid r_{I}\right\}+\operatorname{Pr}\left\{b_{1}=0, b_{2}=1 \mid r_{I}\right\}} \\
& =\frac{f\left(r_{I} \mid b_{1}=1, b_{2}=0\right)+f\left(r_{I} \mid b_{1}=1, b_{2}=1\right)}{f\left(r_{I} \mid b_{1}=0, b_{2}=0\right)+f\left(r_{I} \mid b_{1}=0, b_{2}=1\right)} \\
\mathrm{LR}_{b_{1}}^{\text {init }} & =\frac{\exp \left(\frac{-1}{N_{0}}\left(r_{I}-3 \alpha A\right)^{2}\right)+\exp \left(\frac{-1}{N_{0}}\left(r_{I}-\alpha A\right)^{2}\right)}{\exp \left(\frac{-1}{N_{0}}\left(r_{I}+3 \alpha A\right)^{2}\right)+\exp \left(\frac{-1}{N_{0}}\left(r_{I}+\alpha A\right)^{2}\right)} .
\end{aligned}
$$

Applying the same procedure to the remaining bits will yield the following LRs of $b_{2}$, $b_{3}$ and $b_{4}$, respectively

$$
\begin{aligned}
\mathrm{LR}_{b_{2}}^{\text {init }} & =\frac{\exp \left(\frac{-1}{N_{0}}\left(r_{I}+\alpha A\right)^{2}\right)+\exp \left(\frac{-1}{N_{0}}\left(r_{I}-\alpha A\right)^{2}\right)}{\exp \left(\frac{-1}{N_{0}}\left(r_{I}+3 \alpha A\right)^{2}\right)+\exp \left(\frac{-1}{N_{0}}\left(r_{I}-3 \alpha A\right)^{2}\right)} \\
\mathrm{LR}_{b_{3}}^{\text {init }} & =\frac{\exp \left(\frac{-1}{N_{0}}\left(r_{Q}-3 \alpha A\right)^{2}\right)+\exp \left(\frac{-1}{N_{0}}\left(r_{Q}-\alpha A\right)^{2}\right)}{\exp \left(\frac{-1}{N_{0}}\left(r_{Q}+3 \alpha A\right)^{2}\right)+\exp \left(\frac{-1}{N_{0}}\left(r_{Q}+\alpha A\right)^{2}\right)}
\end{aligned}
$$




$$
\mathrm{LR}_{b_{4}}^{\text {init }}=\frac{\exp \left(\frac{-1}{N_{0}}\left(r_{Q}+\alpha A\right)^{2}\right)+\exp \left(\frac{-1}{N_{0}}\left(r_{Q}-\alpha A\right)^{2}\right)}{\exp \left(\frac{-1}{N_{0}}\left(r_{Q}+3 \alpha A\right)^{2}\right)+\exp \left(\frac{-1}{N_{0}}\left(r_{Q}-3 \alpha A\right)^{2}\right)}
$$

Neal's message-passing algorithm [46] is then used to calculate the LRs of the code bits to decode the code.

\subsubsection{Type II HARQ}

This research work adopts type-II hybrid ARQ, using the RC/QC-LDPC code. Type-II HARQ is found efficient in saving resources and increasing the total system transmission throughput. The information data blocks are first CRC padded, to produce information messages $\boldsymbol{u}$ of length $K$. The LDPC encoder encodes the messages at the lowest code rate $R_{\text {min }}$; generating all needed parity bits to communicate assuming the worst expected channel conditions. The maximum codeword length is $N_{\max }=\frac{K}{R_{\min }}$. The transmitter will partition the codeword into packets. The first packet is the longest, of length $K$. The remaining packets are of a constant preselected packet size $L_{\text {inc }}$.

With the type II HARQ protocol adopted here, as the transmitter starts transmitting, the decoder collects data and starts decoding after receiving the first packet. If decoding is successful, the receiver will send back a positive acknowledgment (ACK) indicating a successful transmission. If the decoding is not successful, then the receiver will not send any acknowledgement, but will wait until the next packet is received before attempting to decode again. The transmitter continues transmitting additional parity packets, until either receiving a positive acknowledgment or the maximum time $\left(t_{\max }\right)$ for transmission is exceeded. If a given channel operates at a transmission rate of $W$, then 
$t_{\max }$ is slightly greater than $N_{\max } / W$ taking into account the round-trip delay and decoder processing time. If no acknowledgments are sent by the receiver until $t_{\text {max }}$ has elapsed, then the transmission is considered "failed". In this case the receiver is deemed "un-servable", corresponding to an outage event. Although outages are undesirable, spending more time trying to serve this user will degrade the performance of the other users of the system, which is also undesirable.

\subsection{Single-Hop Throughput Performance}

This section presents the single-hop throughput performance of the system described in the last section. The performance indication is the average throughout, which is defined as the ratio of the number of received information bits to the number of channel uses (number of transmissions) required to successfully convey the information to the receiver. This is given as

$$
\eta=\frac{\# \text { of } \text { information bits }}{\# \text { of transmissions }}
$$

Since the number of CRC parity bits is small compared to the number information bits $(K)$, the CRC bits are ignored in the throughout calculation. Also, the impact of using type II HARQ messages are ignored in the throughput calculation, along with the effects of the non-zero round trip delay and decoder processing times. For the BPSK modulation, every transmitted signal carries $n_{d}=1$ code bit, while the 16-QAM modulation signal carries $n_{d}=4$ code bits in each transmission. If $K$ is the number of information bits, and $N_{c}(i)$ is the number of code bits required to successfully decode information message $i$, then, the throughput is calculated as 


$$
\eta=n_{d} \frac{T K}{\sum_{i}^{T} N_{c}(i)}
$$

where $T$ is the total number of transmitted information messages of length $K$.

In this work, two sizes of information messages are considered: $K=384$ and $K=1536$ (these lengths are chosen to avoid the zero padding, a technique used for the 3GPP2 codes). Table 2.1 shows the specification of the RC/QC-LDPC code for these two cases.

\begin{tabular}{|l|c|c|}
\hline Parameter & $\mathbf{K = 3 8 4}$ & $\mathbf{K = 1 5 3 6}$ \\
\hline Incremental step $L_{\text {inc }}$ & 16 & 64 \\
\hline$N_{\min }$ & 400 & 1600 \\
\hline$N_{\max }$ & 3840 & 15360 \\
\hline Punctured State bits $Z_{B}$ & $c 1 \rightarrow c 192$ & $c 1 \rightarrow c 512 \& c 640 \rightarrow c 768$ \\
\hline
\end{tabular}

Table 2.1: RC/QC-LDPC code specifications for $K=384$ and $K=1536$.

The state bits are the first $K / 2$ bits of the codeword, and 3GPP2 recommends puncturing all these bits. Doing so makes the code stronger, yielding better performance at low-to-moderate SNRs, but makes the code non-systematic, which degrades the performance at high SNRs by limiting the maximum code rate. A brute force search showed that puncturing only 640 of the state bits in the $K=1536$ code, from the positions indicated in Table 2.1, gave the best overall performance.

The encoder and the decoder are simulated based on Neal's LDPC encoder and decoder [46], with modifications to adopt the RC concept and other system criteria at hand. The BPSK and the 16-QAM throughput performances for $K=384$ and $K=1536$, are shown in Figures 2.2-2.5. These figures compare the throughputs of the systems with the capacities of BPSK and 16-QAM [27]. 


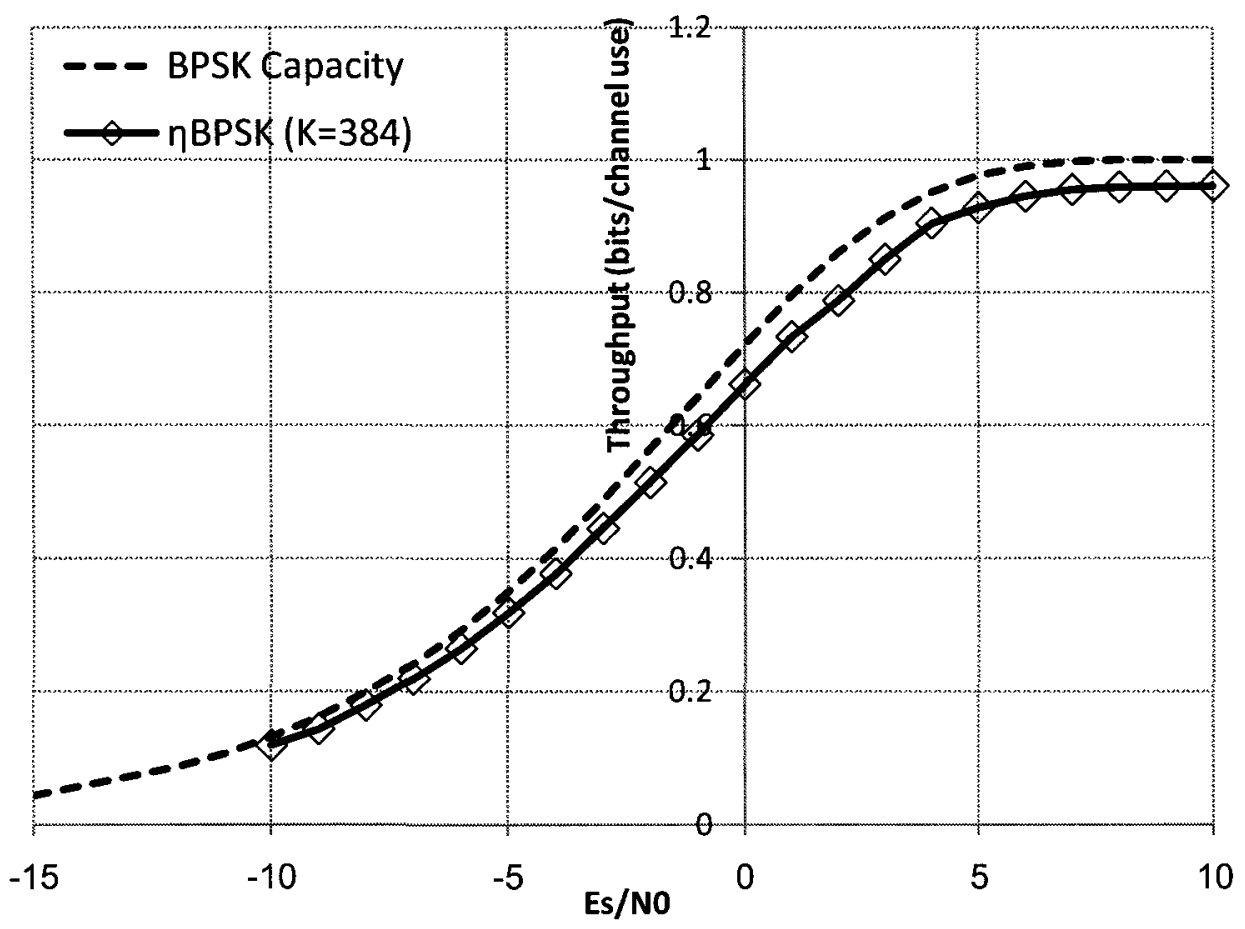

Figure 2.2: RC/QC-LDPC code throughput performance over AWGN channel (BPSK, $K=384)$.

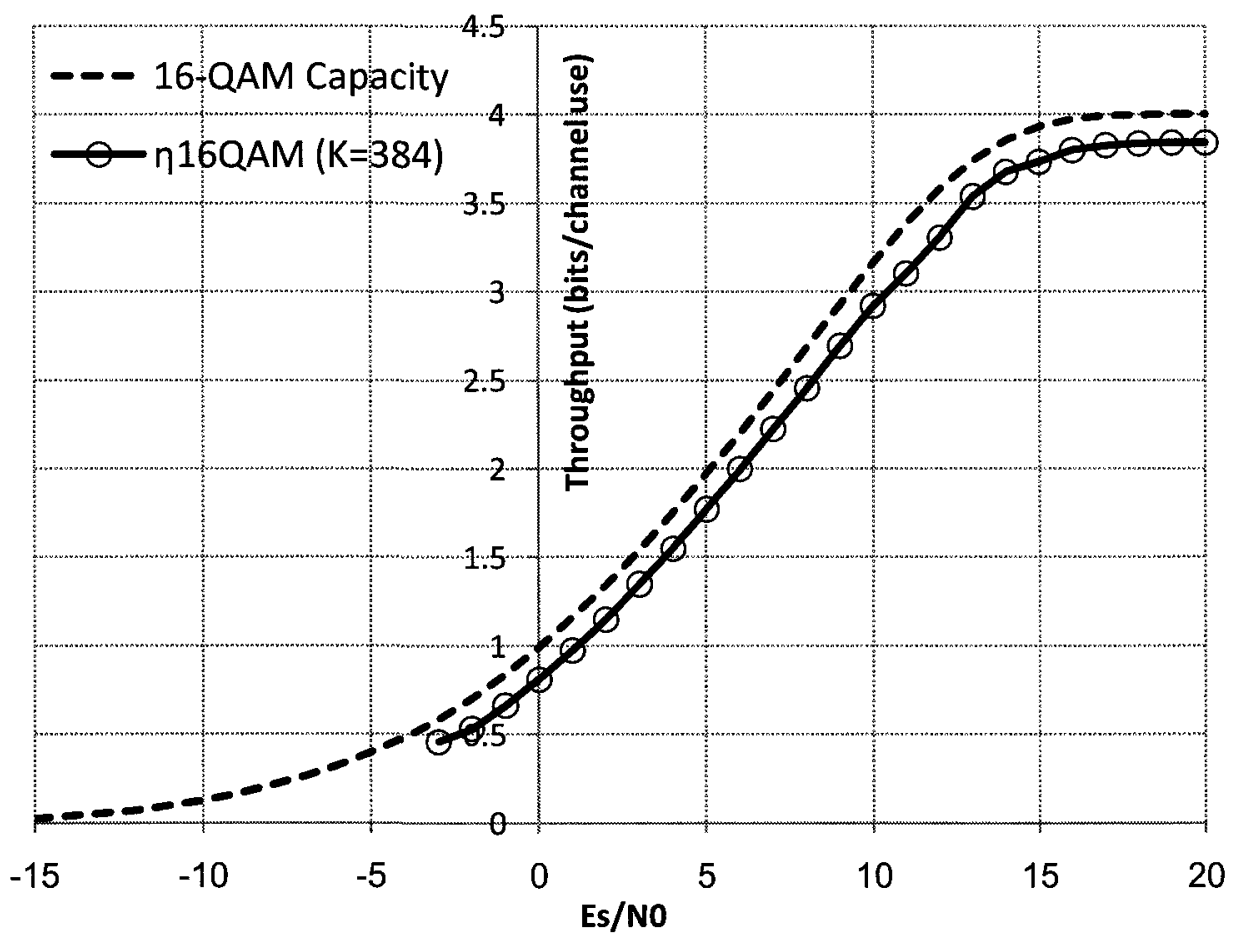

Figure 2.3: RC/QC-LDPC code throughput performance over AWGN channel (16QAM, $K=384)$. 


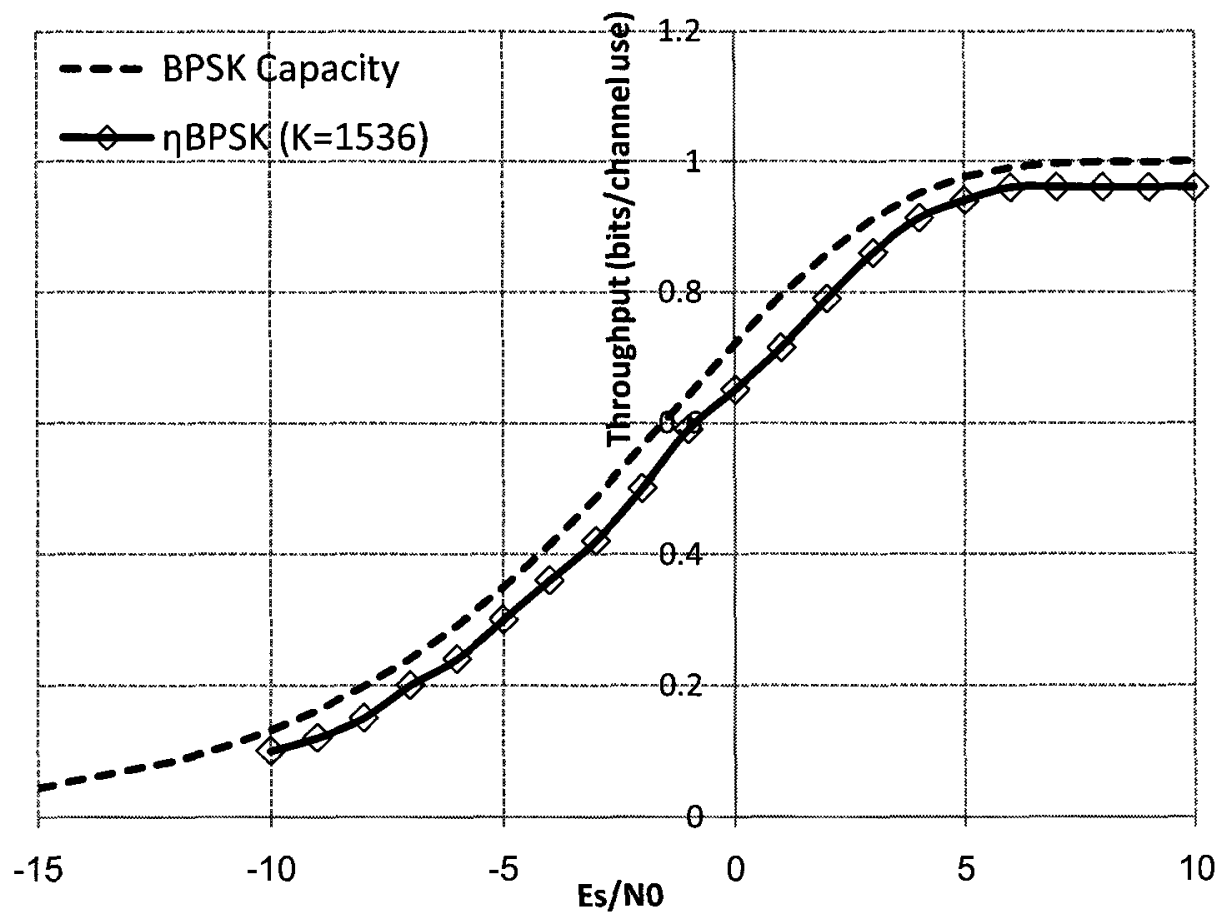

Figure 2.4: RC/QC-LDPC code throughput performance over AWGN channel (BPSK, $K=1536)$.

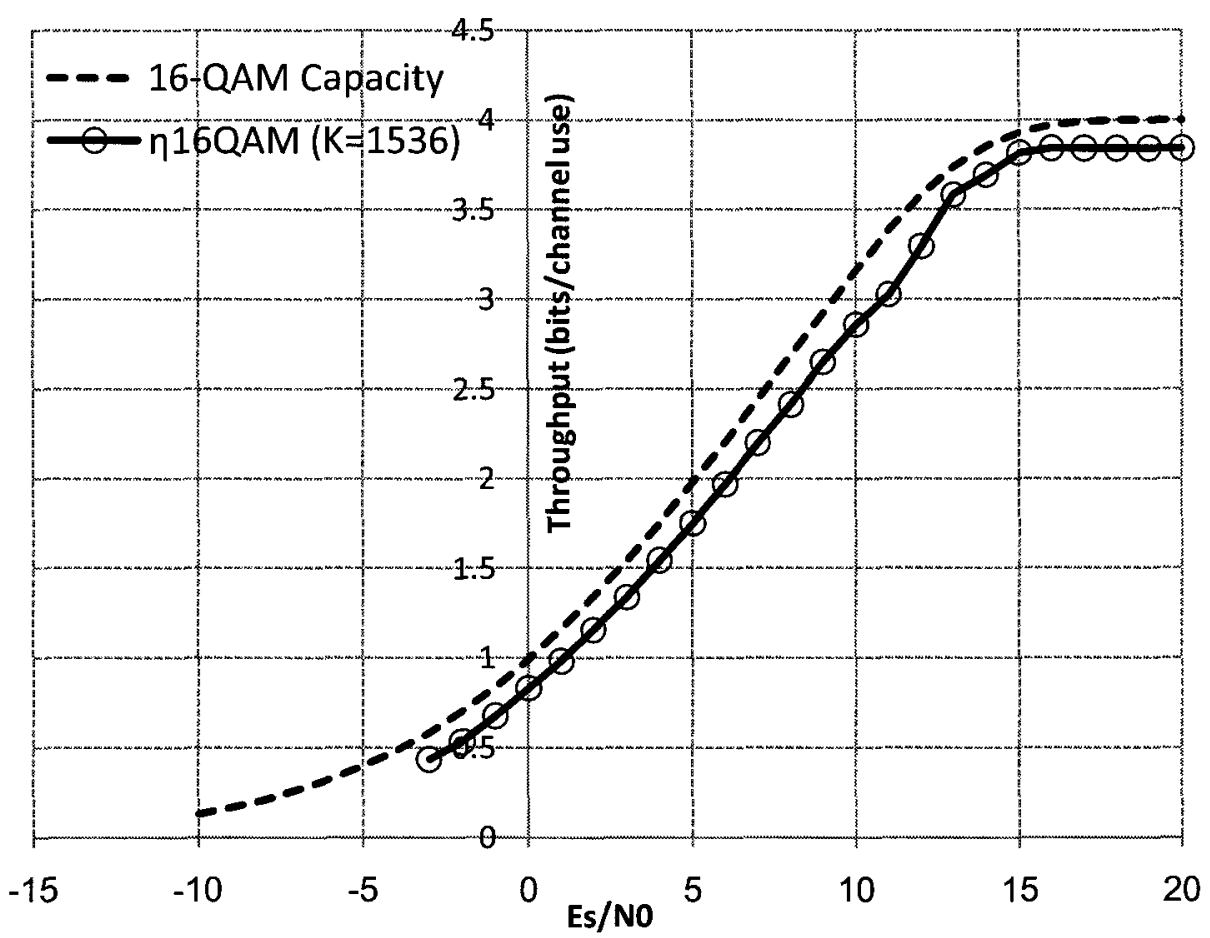

Figure 2.5: RC/QC-LDPC code throughput performance over AWGN channel (16QAM, $K=1536)$. 
The RC/QC-LDPC coding over AWGN channels shows good performance over all. It is apparent that this type of coding comes close to capacity over a wide range of SNRs. It is worth mentioning that the RC/QC-LDPC code used here is extended to lower rates than the work of Haoming $\mathrm{Li}$ in [30]. The reason is that Haoming $\mathrm{Li}$ did not extend the QCLDPC code of 3GPP2, he only used puncturing to convert the code into an RC code. The methods used here in addition to puncturing, are check splitting and combining introduced by Good [47]. Although Good's methods were meant to be applied to irregular-repeat-accumulate (IRA) codes, splitting/combining can also be applied to LDPC codes that are similar in structure to IRA codes.

Table 2.2 shows the comparison of the four scenarios with the respective channel capacities; it shows the SNR gap in dB between the simulated throughputs and their capacities.

\begin{tabular}{|c|c|c|c|c|}
\hline \multirow{2}{*}{ Es/No } & \multicolumn{5}{|c|}{ SNR gap from Channel Capacity (dB) } \\
\cline { 2 - 5 } & BPSK K=384 & BPSK $K=1536$ & 16 -QAM $K=384$ & $16-$ QAM $K=1536$ \\
\hline-10 & 0.53 & 1.25 & & \\
\hline-9 & 0.62 & 1.45 & & \\
\hline-8 & 0.55 & 1.38 & & \\
\hline-7 & 0.52 & 0.96 & & \\
\hline-6 & 0.54 & 1.02 & & \\
\hline-5 & 0.54 & 0.85 & & \\
\hline-4 & 0.58 & 0.84 & & 1.56 \\
\hline-3 & 0.59 & 0.93 & 1.28 & 1.51 \\
\hline-2 & 0.65 & 0.83 & 1.52 & 1.23 \\
\hline-1 & 0.73 & 0.67 & 1.34 & 1.05 \\
\hline 0 & 0.76 & 0.90 & 1.21 & 1.05 \\
\hline+1 & 0.85 & 1.08 & 1.12 & 1.00 \\
\hline+2 & 1.10 & 1.07 & 1.05 & 1.03 \\
\hline+3 & 1.16 & 1.00 & 1.00 & 0.99 \\
\hline+4 & 1.16 & 0.99 & 0.97 & 1.01 \\
\hline+5 & 1.62 & 1.28 & 0.92 & 1.03 \\
\hline+6 & 2.17 & 1.64 & 0.90 & 1.03 \\
\hline+7 & 2.91 & 2.77 & 0.92 & \\
\hline & & & & \\
\hline
\end{tabular}




\begin{tabular}{|c|c|c|c|c|}
\hline+8 & 3.71 & 3.64 & 0.96 & 1.13 \\
\hline+9 & 4.65 & 4.64 & 0.96 & 1.14 \\
\hline+10 & 5.64 & 5.64 & 1.03 & 1.29 \\
\hline+11 & & & 1.26 & 1.63 \\
\hline+12 & & & 1.37 & 1.41 \\
\hline+13 & & & 1.22 & 0.97 \\
\hline+14 & & & 1.39 & 1.27 \\
\hline+15 & & & 2.04 & 1.34 \\
\hline+16 & & & 2.49 & 2.11 \\
\hline+17 & & & 3.25 & 3.11 \\
\hline
\end{tabular}

Table 2.2: SNR gap of the RC/QC-LDPC code from the channel capacities.

\subsection{General Relay System Model}

The general relay system model consists of one relay in addition to the source and the destination. This model is the building block of larger relay networks that might involve multiple relays and multiple destinations, where each destination is associated with one relay at a time. In multi-relay networks, the relay association is communicated through the type II HARQ communication channel which is assumed always reliable and available. Type II HARQ incorporated in relay channels, and relay association is explained first before presenting the operation of the general relay system model.

\subsubsection{Type II HARQ and Relay Association}

When we have relays in the network, type-II HARQ will be slightly modified to take into account the chance of relaying, instead of directly transmitting to the receiver all the time. In this thesis work, it is assumed that relays and end terminals (destinations) share the decision on the relaying option. The destination receives the channel state information (CSI) of the channels directly connected to the destination: the source-todestination (S-D) channel, and the relay-to-destination (R-D) channel. The terms 
"source" and "bases station" are used interchangeably to refer to the same device. It would be possible for the relay to pass the CSI of the source-to-relay $(S-R)$ channel to the destination, but this would introduce more overhead information. In general, this paper assumes the destination has only the CSI of the channels directly connected to it unless stated otherwise.

The acknowledgment messages related to the success of transmission which is sent by either the relay or the destination consist of two parts; the first part is the acknowledgment itself, and the second part is the identification (ID) of the receiver (NACK+ID/ACK+ID), as depicted in Figure 2.6(a).

\begin{tabular}{|l|l|}
\hline ACK/NACK & $R \times 10$ \\
\hline
\end{tabular}

(a) Type II HARQ acknowledgement message

\begin{tabular}{|l|l|l|}
\hline Relaying Mode & Relay ID & RxID \\
\hline
\end{tabular}

(b) Relay Association Message (RelayAssoc)

Figure 2.6: The formats of type II HARQ acknowledgment messages and Relay Association Message.

In addition to the acknowledgment message, our system will adopt one more feedback message called the "Relay Association Message" (RelayAssoc message) which is sent by the destination only. The RelayAssoc message has three parts: the first part describes the relaying mode used, the second message will be the relay-ID that the destination want to use (associate with), and the last part will be the destination-ID. The relay association is based on a forecast calculated by the destination based on the CSI of the channels directly connected to the destination. The RelayAssoc message is made 
general, as if there are as many destinations and relays as required. So, at the beginning of the transmission, training sequences are sent from the transmitters (sources, and relays) to let the receivers (at the relays and the destinations) evaluate the CSI of the direct channels and synchronize to the transmission clock. The general format of acknowledgement messages and RelayAssoc messages are depicted in Figure 2.6(b).

The relaying modes defined in this research belong to three categories: "startover", "combine", and "continue". The way the decoder deals with the three modes will be explained later.

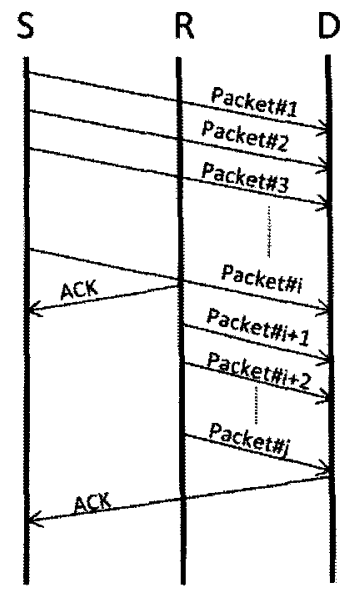

(a) Successful relay transmission

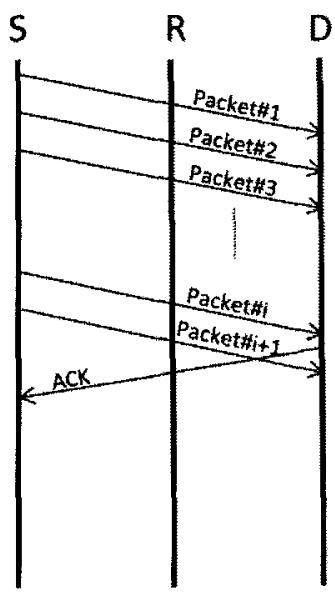

(b) Successful direct transmission

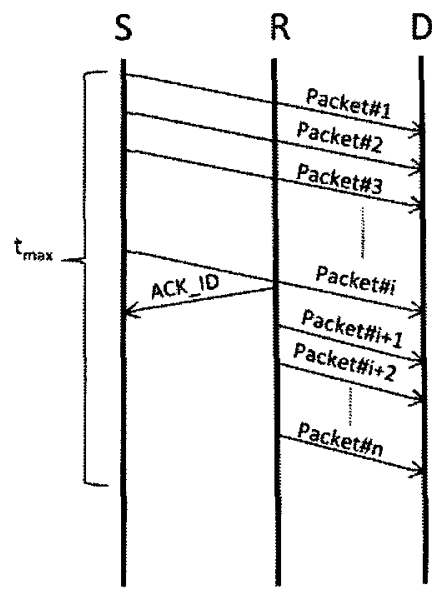

(c) Failed transmission

Figure 2.7: Message flow diagram of type II HARQ applied to relayed-network.

Figure 2.7 depicts the message flow diagram between the three nodes: the source, the destination and the relay. These graphs assume that the relay lies between the source and the destination. This is not necessarily the real situation; it is merely demonstrative.

There are essentially three scenarios for the operation of the relay-type II HARQ. The first scenario, called normal-relaying, is depicted in Figure 2.7(a). The relay is in a 
position that allows it to decode faster than the destination. After the source starts transmitting, the relay and the destination receives some packets of the same codeword. The destination has been updated with the CSI of the S-D and the R-D channels. Then, the destination sends the feedback message RelayAssoc message. Both the relay and the source receive the message successfully, since the feedback channel is assumed to be always reliable. As the relay successfully decodes the codeword before the destination, it sends back an ACK message asking the source to stop the transmission so it can take over. Then the relay starts its transmission based on the RelayAssoc message sent by the destination. The relay continues the transmission until the destination sends back a positive acknowledgment.

The second scenario called the direct-transmission is depicted in Figure $2.7(b)$. In this case the destination decodes the codeword before the relay, so relaying does not occur. Still, the destination may have sent the RelayAssoc message, though it was not useful to the transmission.

The third scenario called transmission outage is depicted in Figure 2.7(c). If the transmission (either relayed or direct) exceeds the preset maximum time $t_{\max }$, then the system is experiencing an outage.

\subsubsection{General Relay System Operation and Relaying Modes}

A block diagram of the general relay model is depicted in Figure 2.8. The figure assumes that the destination has already associated with a relay. 


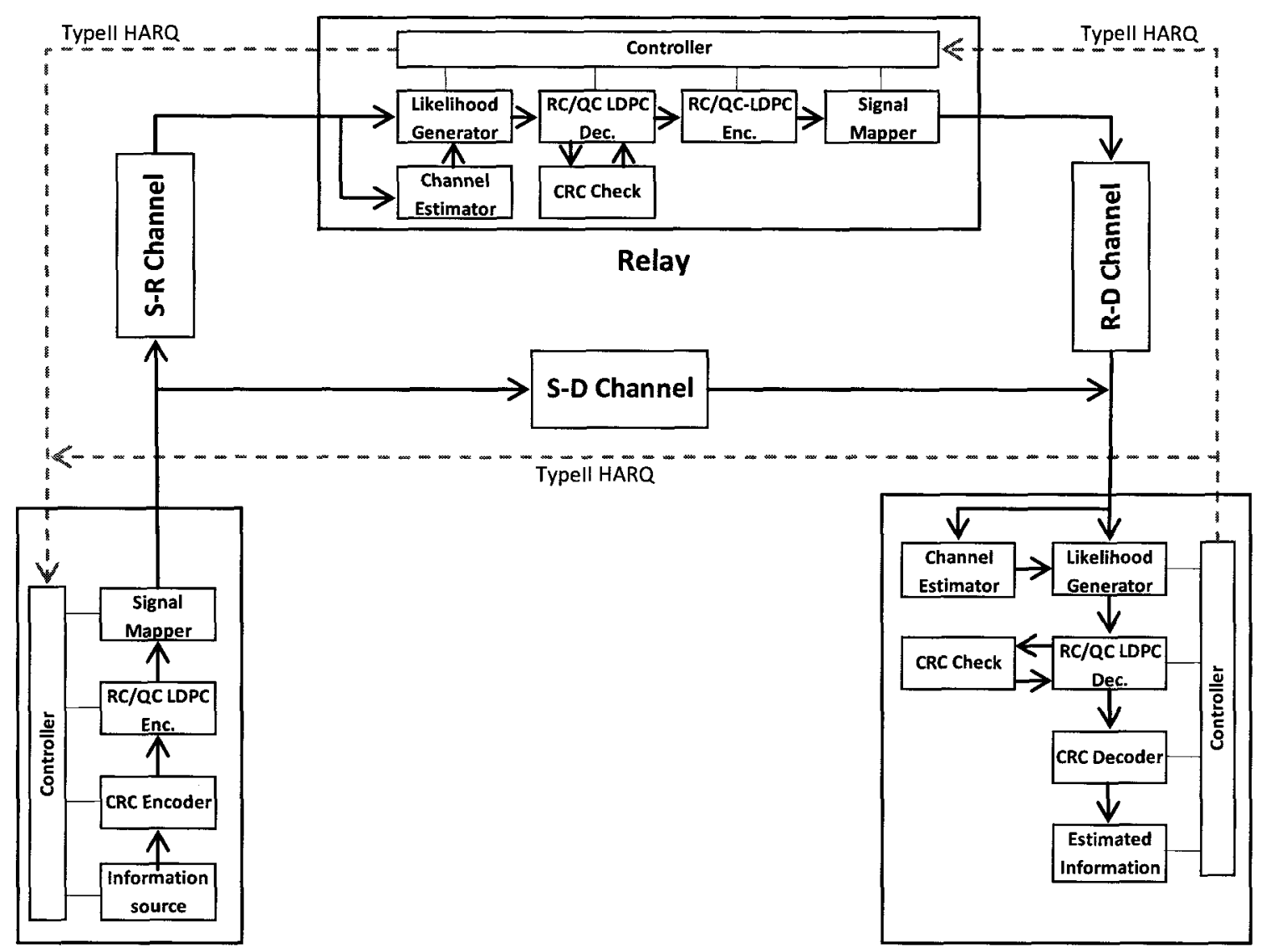

Source

Destination

Figure 2.8: General relay model.

As the source receives the information data, it adds CRC bits to the original message, encodes the message using the RC/QC-LDPC code, maps the code bits into modulation (BPSK or 16-QAM) symbols, and finally transmits the mapped symbols. The transmission will occur with packets of size $L_{\text {inc }}$. The receivers at the destination and the relay start collecting the received samples and generate LRs of the code bits. The channel estimator is used to detect the SNRS of the channels. The RC/QC-LDPC decoders decode the code bits based on the received LRs. The decoded codeword will be periodically checked for correct CRC bits. If a correct CRC check is detected, the codeword is acknowledged as successfully decoded. If the destination decodes successfully before 
the relay does, a positive ACK is sent back and the transmission will stop, freeing up the channel resources used by this transmission session. However, if the relay decodes faster than the destination and the relay is in a good position for better relaying, then the relay will send back an ACK message asking to take over the transmission. The relay will continue the transmission until either the destination successfully decodes the original message or the transmission times out indicating unsuccessful transmission (outage).

The CSIs for the both channels (S-D and R-D) are measured at the destination to decide whether or not to associate with the relay. The case when the relay is considered to be in a good position is when the R-D channel has a larger SNR than the S-D channel. The CSIs of the R-D channel and the S-D channel are enough to predict which would be faster: continuing with the current transmission from the source or switching to the relay.

Acknowledgment messages from relays and destinations are distinguished by the terminal ID. As described in the type II HARQ section, end terminals search for the best relays in the network based on the CSI and consequently broadcast their relayassociation preferences over the uplink channel via the message RelayAssoc.

Time Division Duplex (TDD) is used here to prevent the relay from transmitting and receiving simultaneously. Furthermore, TDD can operate in two different modes: simultaneous and non-simultaneous relaying. Simultaneous TDD relaying dictates that the source and the relay may transmit simultaneously after the relay picks up the original message while non-simultaneous TDD relaying dictates that the source 
transmitter stops once the relay picks up the original message. The one relay model described here uses non-simultaneous TDD relaying. So when the relay starts transmitting, the source stops for the remainder of the time window.

Based on the relaying mechanism and how the end wireless terminal makes use of the received samples from the relay, there are three modes of relaying: startover, continue, and combine. In the startover mode, when the relay successfully decodes the original message before the end terminal, the relay starts over, sending the code bits from the beginning of the codeword. The destination decoder just dumps all previously received samples and starts collecting new samples from the relay. In this mode, the previously sent samples from the source are not utilized by any means. Startover is also known as "cascade" or "multihop" relaying.

The combine and continue relaying modes are examples of "cooperative" relaying, since the source and the destination cooperate in their transmissions. As for the combine relaying mode, the relay works exactly the same way as in startover mode. However, the destination decoder stores the old samples from the source and combines them with the new samples, ostensibly to enhance the decoding process. The destination decoder only combines the repeated portions of the codeword being resent by the relay. If decoding is not successful after receiving all repeated code bits, the relay will send extra code bits that haven't been sent by the source. So, the destination will only combine duplicate code bits before decoding while induplicate code bits will be treated normally. 
For the continue relaying mode, the relay knows where the source stops and continues sending data as if no data flow disturbance has occurred. The decoder appends the samples from the relay to the samples previously sent by the source and decodes normally.

The LRs are calculated based on the SNRs of the channels which are extracted by the channel estimator. Consequently, the LRs of the data sent by the source are computed using the SNR of the S-D channel and LRs of the data sent by the relay are computed based on the SNR of the R-D channel. In the cases of the startover and the continue modes, LRs are found straightforward. However for the combine mode, LRs need additional work.

The samples at the destination received from the source directly and from the relay are respectively given as

$$
\begin{aligned}
& r_{S D, i}=\alpha_{S D} s_{i}+w_{S D, i} \\
& r_{R D, i}=\alpha_{R D} s_{i}+w_{R D, i}
\end{aligned}
$$

and the received samples at the relay are given as

$$
r_{S R, i}=\alpha_{S R} s_{i}+w_{S R, i}
$$

Because non-simultaneous TDD relaying is used, at any time either $r_{S D, i}$ or $r_{R D, i}$ is received at the destination. In the general case, when LRs are computed on one received sample (not considering combining two signals), the LR of bit $c_{n}$ is computed as

$$
\begin{aligned}
\mathrm{LR}_{S D}^{\text {init }} & =\frac{f\left(r_{S D, i} \mid c_{n}=1\right)}{f\left(r_{S D, i} \mid c_{n}=0\right)} \\
\mathrm{LR}_{R D}^{\text {init }} & =\frac{f\left(r_{R D, i} \mid c_{n}=1\right)}{f\left(r_{R D, i} \mid c_{n}=0\right)} .
\end{aligned}
$$


These formulas are used for the startover mode, the continue mode and also for code bits in the combine mode which are not repeated. However for those code bits in the combine mode which have two signals repeated from the source and the relay, the LR is different. The LR values for repeated code bit signals are derived as follows

$$
\begin{aligned}
\mathrm{LR}_{S D+R D}^{\text {init }} & =\frac{f\left(r_{R D, i}, r_{S D, i} \mid c_{n}=1\right)}{f\left(r_{R D, i}, r_{S D, i} \mid c_{n}=0\right)}=\frac{f\left(r_{S D, i} \mid c_{n}=1\right)}{f\left(r_{S D, i} \mid c_{n}=0\right)} \frac{f\left(r_{R D, i} \mid c_{n}=1\right)}{f\left(r_{R D, i} \mid c_{n}=0\right)} \\
& =\mathrm{LR}_{S D}^{\text {init }} \mathrm{LR}_{R D}^{\text {init }} .
\end{aligned}
$$

So, whenever combining two LRs of a code bit, their combined value is their product. Alternatively, if working with LLRs, the combined LLR is their sum.

\subsection{Linear Relay System Model}

The main goal here is to analyze the efficiency of relaying when using the relaying modes (startover, continue and combine) with rateless coding. In general, relaying is not always required. In fact, relaying is needed only if the S-D channel is weaker than R-D channel. A practical approach to force relaying is when the source, the relay and the destination are aligned and the relay is always between the source and the destination.

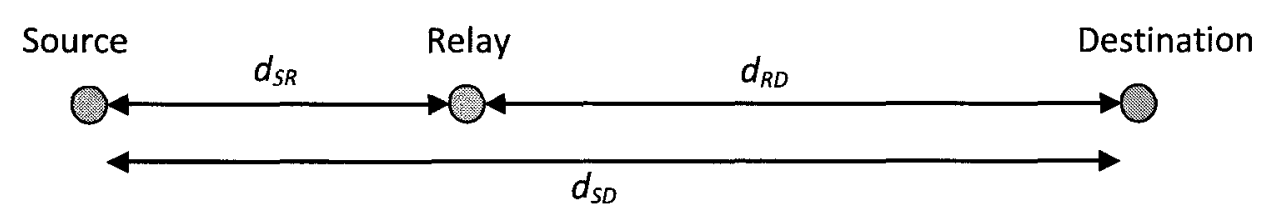

Figure 2.9: Linear relay system model.

Figure 2.9 depicts the linear relaying system model. The distance between the source and the destination is $d_{S D}$. The relay is positioned at distance $d_{S R}=\mu d_{S D}$ from the 
source, and $d_{R D}=(1-\mu) d_{S D}$ from the destination, where $\mu \in(0,1)$ is the relative relay position. In this model, we only consider the pathloss and the AWGN impairments. The pathloss factors of the three channels will be captured by $\alpha_{S D}^{2}, \alpha_{S R}^{2}$, and $\alpha_{R D}^{2}$ only. To keep things simple, it is assumed that $\alpha_{S D}^{2}, \alpha_{S R}^{2}$, and $\alpha_{R D}^{2}$ depend only on the distances between the three elements and the pathloss exponent $\left(n_{p}\right)$, so the pathloss factors are computed as follows

$$
\begin{aligned}
& \alpha_{S D}^{2}=\left(\frac{1}{d_{S D}}\right)^{n_{p}} \\
& \alpha_{S R}^{2}=\left(\frac{1}{d_{S R}}\right)^{n_{p}}=\mu^{-n_{p}} \alpha_{S D}^{2} \\
& \alpha_{R D}^{2}=\left(\frac{1}{d_{R D}}\right)^{n_{p}}=(1-\mu)^{-n_{p}} \alpha_{S D}^{2} .
\end{aligned}
$$

Hence, the respective SNRs of the three channels are given as

$$
\begin{aligned}
& \gamma_{S D}=10 \log \left(\frac{\alpha_{S D}^{2} E_{S}}{N_{0}}\right) \\
& \gamma_{S R}=10 \log \left(\frac{\alpha_{S R}^{2} E_{S}}{N_{0}}\right)=\gamma_{S D}-10 n_{p} \log \mu \\
& \gamma_{R D}=10 \log \left(\frac{\alpha_{R D}^{2} E_{S}}{N_{0}}\right)=\gamma_{S D}-10 n_{p} \log (1-\mu) .
\end{aligned}
$$

Although it is possible, and for certain scenarios necessary, to let the source and the relay transmit at different power levels, here they are assumed to have the same transmission power. 


\subsection{Theoretical Relay Performance}

Most of the researches use capacity (or data rate) when studying relay networks and do not talk about the throughput explicitly. As a matter of fact, data rate and throughput are not always the same. With full-duplex relaying and simultaneous TDD/FDD relaying, data rate and throughput are different. In these cases, the data rate is the number of message bits divided by simultaneous channel uses. On the other hand, the throughput is the number of message bits divided by the total sum of the source's channel uses and the relay's channel uses. In the non-simultaneous TDD/FDD relaying where only either the source or the relay is allowed to transmit, the data rate is equal to the throughput.

Before proceeding with the theoretical performance of the relay methods, the analyses here assume the following:

1) Rateless coding is used.

2) Transmission is assumed to be non-simultaneous TDD, so there is no simultaneous transmission from the source and the relay.

3) As soon the relay has enough information to contribute to the system the remaining portion of the channel will be used by the relay.

4) Single-hop throughput performance behavior versus the channel SNR is known and predictable.

5) The channel impairments used here are the AWGN and path losses.

Since the single-hop throughput is a function of the channel SNR $(\eta=F(\mathrm{SNR}))$, then the three channels in the relay system model have the following throughputs 


$$
\begin{aligned}
& \eta_{S D}=F\left(\gamma_{S D}\right) \\
& \eta_{S R}=F\left(\gamma_{S R}\right) \\
& \eta_{R D}=F\left(\gamma_{R D}\right) .
\end{aligned}
$$

Since it is assumed that the source is always closer to the relay than it is to the destination, the relay decodes faster than the destination. For the startover relaying mode, the system works as a cascade relay system (multihop). The relay decodes the message faster than the destination and starts over from the beginning, sending the code bits to the destination. The destination in this mode does not keep the previously received code bits from the source; it rather clears off its buffers and starts decoding whatever is received from the relay only. The startover throughput can be calculated as [4]

$$
\eta_{\text {startover }}=\left(\sum_{i=1}^{N_{\text {hop }}=2} \frac{1}{\eta_{i}}\right)^{-1}=\left(\frac{1}{\eta_{S R}}+\frac{1}{\eta_{R D}}\right)^{-1}=\frac{\eta_{S R} \eta_{R D}}{\eta_{S R}+\eta_{R D}}
$$

where $i$ is the hop number, and $N_{\text {hop }}$ is the total number of hops which in this case is equal to 2 . Notice that if the S-D channel is stronger than the R-D channel then the relaying will not happen and the throughput is equal to the direct-transmission throughput: $\eta_{\text {startover }}=\eta_{S D}$.

However for the continue relaying mode, the result is different. It is because the destination uses previously sent code bits from the source. Starting with the first hop (SR), the average number of code bits required for the relay to decode the transmitted codeword is given as

$$
N_{S R}=\frac{K}{\eta_{S R}} .
$$


By the time the relay decodes the message successfully, the destination has already received $K_{1}$ bits of useful information, which is calculated as

$$
K_{1}=N_{S R} \eta_{S D}
$$

But the destination still requires additional $K_{2}$ bits of information to be able to decode successfully. $K_{2}$ is computed as follows

$$
K_{2}=K-K_{1}=K-N_{S R} \eta_{S D}
$$

The remaining required information is going to be transmitted by the relay. To receive $K_{2}$ bits of information from the relay, the destination requires $N_{R D}$ code bits, where

$$
N_{R D}=\frac{K_{2}}{\eta_{R D}}=\frac{K-N_{S R} \eta_{S D}}{\eta_{R D}}
$$

Because the total number of transmissions required to convey $K$ bits of information is $N_{S R}+N_{R D}$, the effective throughout of continue relaying is

$$
\begin{aligned}
\eta_{\text {continue }} & =\frac{K}{N_{S R}+N_{R D}}=\frac{K}{\frac{K}{\eta_{S R}}+\frac{K-N_{S R} \eta_{S D}}{\eta_{R D}}}=\frac{K}{\frac{K}{\eta_{S R}}+\frac{K}{\eta_{R D}}-\frac{K \eta_{S D}}{\eta_{S R} \eta_{R D}}} \\
& =\frac{\eta_{S R} \eta_{R D}}{\eta_{S R}+\eta_{R D}-\eta_{S D}} .
\end{aligned}
$$

The formula is very similar to the startover relaying throughput except for the term $\left(-\eta_{S D}\right)$ in the denominator. This term will increase the relaying throughput, which is an expected result since the destination overhears the source transmissions while the relay is decoding. The simulation results presented in the next chapter confirm that the above formula correctly predicts the relay channel behavior. 
With combine relaying, transmission occurs in three distinct phases. In the first phase, the source is transmitting code bits, and information is being received at the destination at a rate of $\eta_{S D}$ bits per channel use. This phase lasts for $N_{S R}$ channel uses. The total amount of information received up to and including the $i^{\text {th }}$ channel use is

$$
K_{i}=i \eta_{S D}
$$

for $1 \leq i \leq N_{S R}$

In the second phase, the relay retransmits previously transmitted code bits. The received samples from the relay are combined with the previously received samples from the source, effectively improving the SNR. The effective SNR of the combined samples is $\gamma_{C}=\gamma_{S D}+\gamma_{R D}$, where the addition is performed in the linear domain (not in $\mathrm{dB})$. The amount of information contained in each combined sample is $\eta_{\mathrm{C}}=F\left(\gamma_{C}\right)$. This second phase lasts for an additional $N_{S R}$ channel uses, until all previously transmitted code bits have been resent. For $N_{S R}+1 \leq i \leq 2 N_{S R}$, the amount of information received up to the $i^{\text {th }}$ channel use is

$$
K_{i}=\left(i-N_{S R}\right) \eta_{C}+\left(2 N_{S R}-i\right) \eta_{\mathrm{SD}}
$$

where the first term corresponds to information in the combined samples, and the second term corresponds to the information in those samples that have so far only been transmitted by the source.

In the third phase the relay begins transmitting new code bits. Each received sample contains $\eta_{\mathrm{RD}}$ bits of information, so the total amount of information received up to the $i^{\text {th }}$ channel use (for $i>2 N_{S R}$ ) is

$$
K_{i}=N_{S R} \eta_{C}+\left(i-2 N_{S R}\right) \eta_{\mathrm{RD}}
$$


where the first term corresponds to the information contained in all the combined samples, and the second term corresponds to the information in the induplicate samples from just the relay.

In summary, the total amount of information received at the destination after the $i^{\text {th }}$ channel use is

$$
K_{i}=\left\{\begin{array}{lr}
i \eta_{S D} & 1 \leq i \leq N_{S R} \\
\left(i-N_{S R}\right) \eta_{C}+\left(2 N_{S R}-i\right) \eta_{S D} & N_{S R}+1 \leq i \leq 2 N_{S R} \\
N_{S R} \eta_{C}+\left(i-2 N_{S R}\right) \eta_{R D} & i>2 N_{S R}
\end{array}\right.
$$

The destination is assumed to correctly receive the message as soon as it collects $K$ bits of information. To determine how many channel uses are required until this occurs, we need to find $i$ such that $K_{i}=K$. This depends on whether the $K$ information bits are finally received during the second phase or the third phase (because $\gamma_{S D}<\gamma_{S R}$, the relay always receives the message before the destination, so we can ignore the first phase).

The total amount of information received at the end of the first phase is $N_{S R} \eta_{S D}$, and at the end of the second phase is $N_{S R} \eta_{C}$. So, the full $K$ bits are received during the second phase if $N_{S R} \eta_{S D}<K \leq N_{S R} \eta_{C}$. Since $N_{S R}=\frac{K}{\eta_{S R}}$, this is equivalent to $\frac{\eta_{S D}}{\eta_{S R}}<1 \leq$ $\frac{\eta_{C}}{\eta_{S R}}$, or $\eta_{S D}<\eta_{S R} \leq \eta_{C}$

Setting $K_{i}=K$ and solving for $i$ gives

$$
\left(i-N_{S R}\right) \eta_{C}+\left(2 N_{S R}-i\right) \eta_{S D}=K
$$

or

$$
i=\frac{K+N_{S R} \eta_{C}-2 N_{S R} \eta_{S D}}{\eta_{C}-\eta_{S D}}
$$


Substituting $N_{S R}=\frac{\mathrm{K}}{\eta_{S R}}$ gives

$$
i=\frac{K}{\eta_{S R}} \frac{\eta_{S R}+\eta_{C}-2 \eta_{S D}}{\eta_{C}-\eta_{S D}}
$$

as the total number of channel uses required to transmit $K$ bits of information. The overall throughput is therefore

$$
\eta_{\text {combine } 1}=\frac{K}{i}=\frac{\eta_{S R}\left(\eta_{C}-\eta_{S D}\right)}{\eta_{S R}+\eta_{C}-2 \eta_{S D}}
$$

However, if $K>N_{S R} \eta_{C}$ (or equivalently $\eta_{S R}>\eta_{C}$ ) then the full $K$ bits are received during the third phase. Setting $K_{i}=K$ and solving for $i$ gives

$$
N_{S R} \eta_{C}+\left(i-2 N_{S R}\right) \eta_{R D}=K
$$

or

$$
i=\frac{K}{\eta_{S R}} \frac{\eta_{S R}+2 \eta_{R D}-\eta_{C}}{\eta_{R D}}
$$

The overall throughput in this case is

$$
\eta_{\text {combine } 2}=\frac{K}{i}=\frac{\eta_{S R} \eta_{R D}}{\eta_{S R}+2 \eta_{R D}-\eta_{C}}
$$

In summary, the theoretical throughput of combine relaying is

$$
\eta_{\text {combine }}= \begin{cases}\frac{\eta_{S R}\left(\eta_{C}-\eta_{S D}\right)}{\eta_{S R}+\eta_{C}-2 \eta_{S D}} & \text { if } \eta_{S R} \leq \eta_{C} \\ \frac{\eta_{S R} \eta_{R D}}{\eta_{S R}+2 \eta_{R D}-\eta_{C}} & \text { if } \eta_{S R}>\eta_{C}\end{cases}
$$

\subsection{Generalization of Results to Triangular Relaying}

Linear relaying only tackles a special case of a more general one, which is the triangular relaying. Figure 3.34 depicts the three general triangular relaying models. The 
two criteria that define the three general relay cases are: the First Successful Decoder (FSD), and the Better Direct Link with Destination (BDLD). FSD specifies which of the two decoders (the destination or the relay) decodes faster, while BDLD specifies which direct link with the destination is better (whether it be the S-D channel or the R-D channel).

(a) Case 1:

$\mathrm{FSD}=$ Relay

$B D L=R-D$

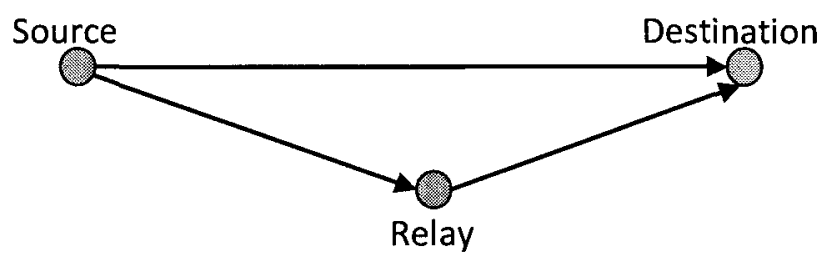

(b) Case 2:

$\mathrm{FSD}=$ Relay

$B D L D=S-D$

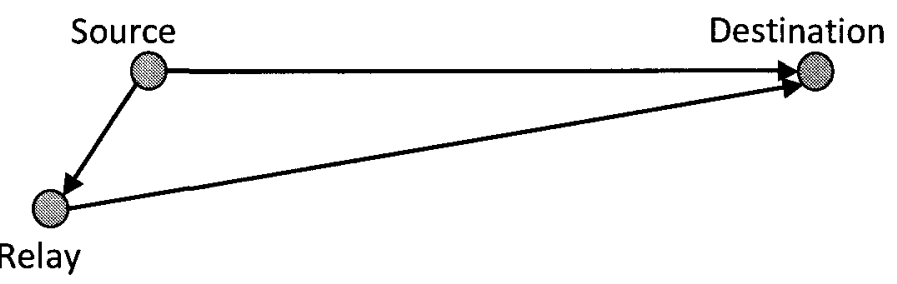

(c) Case 2:

FSD $=$ Destination $B D L D=S-D, R-D$

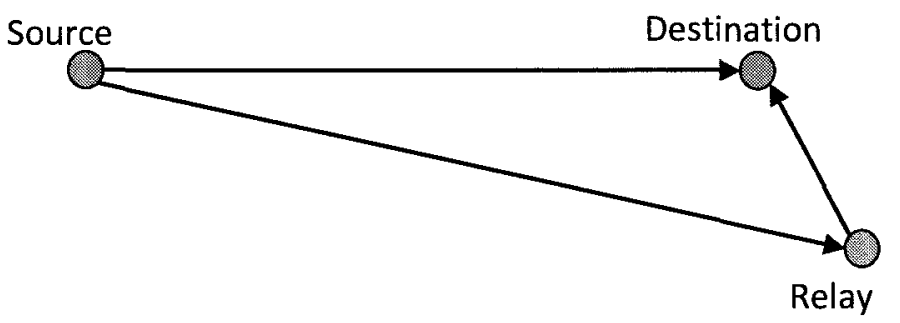

Figure 2.10: Triangular relaying.

The first case is shown in Figure 3.34 (a), which occurs when FSD is the relay $\left(\gamma_{S D}<\gamma_{S R}\right)$ and BDLD is the R-D channel $\left(\gamma_{S D}<\gamma_{R D}\right)$. The linear relay model belongs to this category and relaying is best practiced here. As the destination knows the direct channel CSIs (i.e. S-D and R-D), it decides to use the relay and broadcasts the RelayAssoc message through the uplink channel. As the relay decodes faster, it sends back the ACK message to stop the source transmission and the relay takes over. 
The second case is shown in Figure 3.34 (b), which occurs when FSD is the relay $\left(\gamma_{S D}<\gamma_{S R}\right)$ and BDLD is S-D channel $\left(\gamma_{S D} \geq \gamma_{R D}\right)$. In this situation, it is better not to use the relay, as doing so would degrade the transmission performance. The destination wouldn't associate with the relay by not sending the RelayAssoc message. Only direct single-hop transmission occurs.

The third case is shown in Figure 3.34 (c), which occurs when FSD is the destination $\left(\gamma_{S D} \geq \gamma_{S R}\right)$. Here, the BDLD has no meaningful value since the destination decodes before the relay, so relaying is not needed. Although, the destination may have established association with a relay, the destination decodes faster.

Startover relaying, when applied to the general triangular relay system, only occurs in the first case, where FSD is the relay and the BDLD is the R-D channel. In the general case when the destination has the CSI of only the direct channels and has no information about the CSI of S-R channel, the throughput of a system using the startover relaying is summarized as follows

$$
\eta_{\text {startover-system }}= \begin{cases}\eta_{\text {startover }}=\frac{\eta_{S R} \eta_{R D}}{\eta_{S R}+\eta_{R D}} & \text { if } \gamma_{S D}<\gamma_{S R}, \gamma_{S D}<\gamma_{R D} \\ \eta_{S D} & \text { otherwise }\end{cases}
$$

If the system is more intelligent and passes the CSI of the S-R channel to the destination, the throughput will improve. By having the S-R CSI, the destination can predict the performance of relaying and would decide whether to associate with the relay or not. In this situation, the system throughput would be

$$
\eta_{\text {startover-system }}=\max \left(\eta_{\text {startover }}, \eta_{S D}\right)
$$


Combine relaying perform similar to startover relaying. Hence, if the destination has only the direct channel CSIs (S-D and R-D), then the throughput of combine triangularrelay system would be

$$
\eta_{\text {combine-system }}= \begin{cases}\eta_{\text {combine }} & \text { if } \gamma_{S D}<\gamma_{S R} \text { and } \gamma_{S D}<\gamma_{R D} \\ \eta_{S D} & \text { otherwise }\end{cases}
$$

where $\eta_{\text {combine }}$ is given in Equation (2.43). However if all channel CSIs (S-D, R-D, and SR) are known to the destination, the intelligent combine relaying throughput would be

$$
\eta_{\text {combine-system }}=\max \left(\eta_{\text {combine }}, \eta_{S D}\right)
$$

As for continue relaying, things are less complicated. Continue relaying promises not go below the direct single-hop transmission performance, (i.e. $\eta_{\text {continue }} \geq \eta_{S D}$ ). Since the relay association only occurs if FSD=Relay and BDLD=R-D channel, the system throughput would be the maximum of the relaying throughput and the direct transmission throughput

$$
\eta_{\text {continue-system }}=\max \left(\eta_{\text {continue }}, \eta_{S D}\right)=\max \left(\frac{\eta_{S R} \eta_{R D}}{\eta_{S R}+\eta_{R D}-\eta_{S D}}, \eta_{S D}\right)
$$

The results presented in Chapter 3, which are for linear relaying, also apply to triangular relaying, but only when relay association occurs. 


\section{Simulation Results}

This chapter contains the presentation and the discussion of the simulation results for the linear relay model. In Section 3.1, the simulation parameters are listed. Section 3.2 presents the results of the linear relay model and validates the theoretical prediction of the three types of relaying modes. It also compares the three relaying modes, and concludes with a comparison of the maximum throughout gain of continue and startover relaying versus the single-hop transmission.

\subsection{Simulation Parameters and Description}

The following simulations will find the throughput of the linear relay model. Two message word lengths are considered: short messages ( $K=384$ bits) and long messages $(K=1536$ bits). Message bits are randomly generated using a uniform distribution. The rateless encoder used here is the previously explained RC/QC-LDPC encoder, which has a maximum codeword length $N_{\max }=10 \mathrm{~K}$.

The BPSK symbols assume one of the values in $\{-A,+A\}$ where $A=1$ volt for BPSK. BPSK only uses the I-channel. The average energy per symbol in this case is $E_{\text {avg }}=A^{2}=$ 1 Joule. With 16-QAM the symbols are mapped into one of the values $\{-3 A,-A,+A,+3 A\}$

where $A=\frac{1}{\sqrt{10}}$ volt. The average energy per symbol here is $E_{\text {avg }}=10 A^{2}$ Joule. The additive noise is assumed complex AWGN with $\sigma_{w}^{2}=N_{0}$. The other impairment is the pathloss, which is captured by the value $\alpha$.

The simulations here generate the throughput of the linear relay system model at different transmitted power levels. First, the relaying mode is chosen to be startover, 
combine, or continue. Secondly, the SNR of the S-D is set at a value. Then, the relative relay position $\mu$ will be gradually changed in the range of $(0,1)$. For each relative relay position, the throughput of relaying is computed based on successful decoding at the destination. If $N_{\max }$ bits have been sent and the destination couldn't successfully decode the codeword, then the transmission is said to have timed out (outage).

The signals at the receivers are generated in the following manner:

1- The SNR of the S-D channel is set at a fixed value, $\gamma_{S D}$.

2- The pathloss factors of the three channels are calculated according to Equations $(2.22),(2.23)$, and (2.24):

$$
\begin{aligned}
& \alpha_{S D}^{2}=\frac{N_{0}}{E_{S}} 10^{\frac{\gamma_{S D}}{10}} \\
& \alpha_{S R}^{2}=\mu^{-n_{p}} \alpha_{S D}^{2} \\
& \alpha_{R D}^{2}=(1-\mu)^{-n_{p}} \alpha_{S D}^{2}
\end{aligned}
$$

where $n_{p}$ is the pathloss exponent. The respective SNRs are $\gamma_{S D}, \gamma_{S R}$, and $\gamma_{R D}$.

3- Complex AWGN samples $w_{S D, i}, w_{S R, i}$, and $w_{R D, i}$ are generated independently with variance $\sigma_{w}^{2}=N_{0}$.

4- Based on the generated symbols, the pathloss factors and the AWGN samples, the received samples are computed using Equations (2.13), (2.14), and (2.15).

The LRs of these signals are computed as described in the relay model using Equations (2.16), (2.17), and (2.18), and are passed to the decoders of the relay and to the destination. The RC/QC-LDPC decoders perform a maximum of $l_{\max }=200$ iterations. If decoding is not successful in either decoder, an additional $L_{\text {inc }}$ code bits 
are sent. When the relay decodes the messages successfully (before the destination does), the source stops transmitting and the relay takes over.

The relay will send data based on the relaying mode chosen: startover, combine or continue. The decoder will decode the received code bits based on the relaying mode.

Table 3.1 summarizes the simulation parameters.

\begin{tabular}{|l|c|c|c|}
\hline Parameter & \multicolumn{2}{|c|}{ BPSK } & 16-QAM \\
\hline$K$ & 384 bits & 1536 bits & 384 bits \\
\hline$Z_{B}$ & 192 bits & 640 bits & 192 bits \\
\hline$N_{\text {max }}$ & 3840 bits & 15360 bits & 3840 bits \\
\hline$L_{\text {inc }}$ & 16 bits & 64 bits & 16 bits \\
\hline$n_{p}$ & 3.5 & 3.5 & 3.5 \\
\hline$E_{S}$ & 1 Joule & 1 Joule & 1 Joule \\
\hline$\gamma_{S D}$ & $-6 \mathrm{~dB},-4 \mathrm{~dB},-2 \mathrm{~dB}, 0 \mathrm{~dB}$, & $-6 \mathrm{~dB},-4 \mathrm{~dB},-2 \mathrm{~dB}, 0 \mathrm{~dB}$, & $-2 \mathrm{~dB}, 0 \mathrm{~dB},+2 \mathrm{~dB},+5$ \\
& $+2 \mathrm{~dB},+4 \mathrm{~dB}$ & $+2 \mathrm{~dB},+4 \mathrm{~dB}$ & $\mathrm{~dB},+10 \mathrm{~dB},+15 \mathrm{~dB}$ \\
\hline Relative Relay & $0.01,0.1,0.2,0.25,0.3$, & $0.01,0.1,0.2,0.25,0.3$, & $0.01,0.1,0.2,0.25,0.3$, \\
Position $(\mu)$ & $0.4,0.5,0.6,0.7,0.75$, & $0.4,0.5,0.6,0.7,0.75$, & $0.4,0.5,0.6,0.7,0.75$, \\
& $0.8,0.9,0.99$ & $0.8,0.9,0.99$ & $0.8,0.9,0.99$ \\
\hline Relay Mode & startover, combine, & startover, combine, & startover, continue \\
& continue & continue & 200 \\
\hline$l_{\text {max }}$ & 200 & 200 & \\
\hline
\end{tabular}

Table 3.1: Linear relay model simulation parameters.

For a given relay mode, $\gamma_{S D}$, and $\mu$, the transmission of a total of $T$ message words is simulated. The total number of transmitted code bits required to successfully decode $T$ message words, $\sum_{i}^{T} N(i)$, is counted. The resultant throughputs are calculated as

$$
\eta=n_{d} \frac{T K}{\sum_{i}^{T} N(i)}=n_{d} \frac{T K}{\sum_{i}^{T}\left[N_{S R}(i)+N_{R D}(i)\right]}
$$

where $N_{S R}(i)$ and $N_{R D}(i)$ are the number of code bits transmitted by the source and the relay, respectively, for the $i^{\text {th }}$ message word. 


\subsection{Simulation Results-Linear Relaying}

This section presents the linear-relaying simulation results, discusses them and compares the three relaying modes under different codeword lengths and variable S-D SNRs. It also compares the theoretical throughput performances against the actual throughput performances.

\subsubsection{Simulation Results for $K=1536$ and BPSK}

Starting with $K=1536$ and BPSK, Figure 3.1 compares the actual throughput performance of the startover relaying mode with its theoretical prediction (given by Equation (2.28)). This figure shows clearly the exact matching between the theoretical and the actual throughput performances at different S-D SNRs. Actually this validation was expected because the startover relaying is simple and easily predicted.

It is worth noticing that the startover performance improves as the S-D SNR increases, and saturates to its maximum value of 0.48 bits/channel use. The startover throughput saturates when the S-R link and R-D link are in their best conditions; that is when, the S-R link and R-D link can operate at the maximum throughput for single-hop transmission (0.96 bits/channel use). Note also that the maximum relay throughput occurs at the mid distance between the source and the destination. As the S-D SNR increases, the relay throughput becomes more stable around the mid distance and the maximum throughput performance region broadens. 


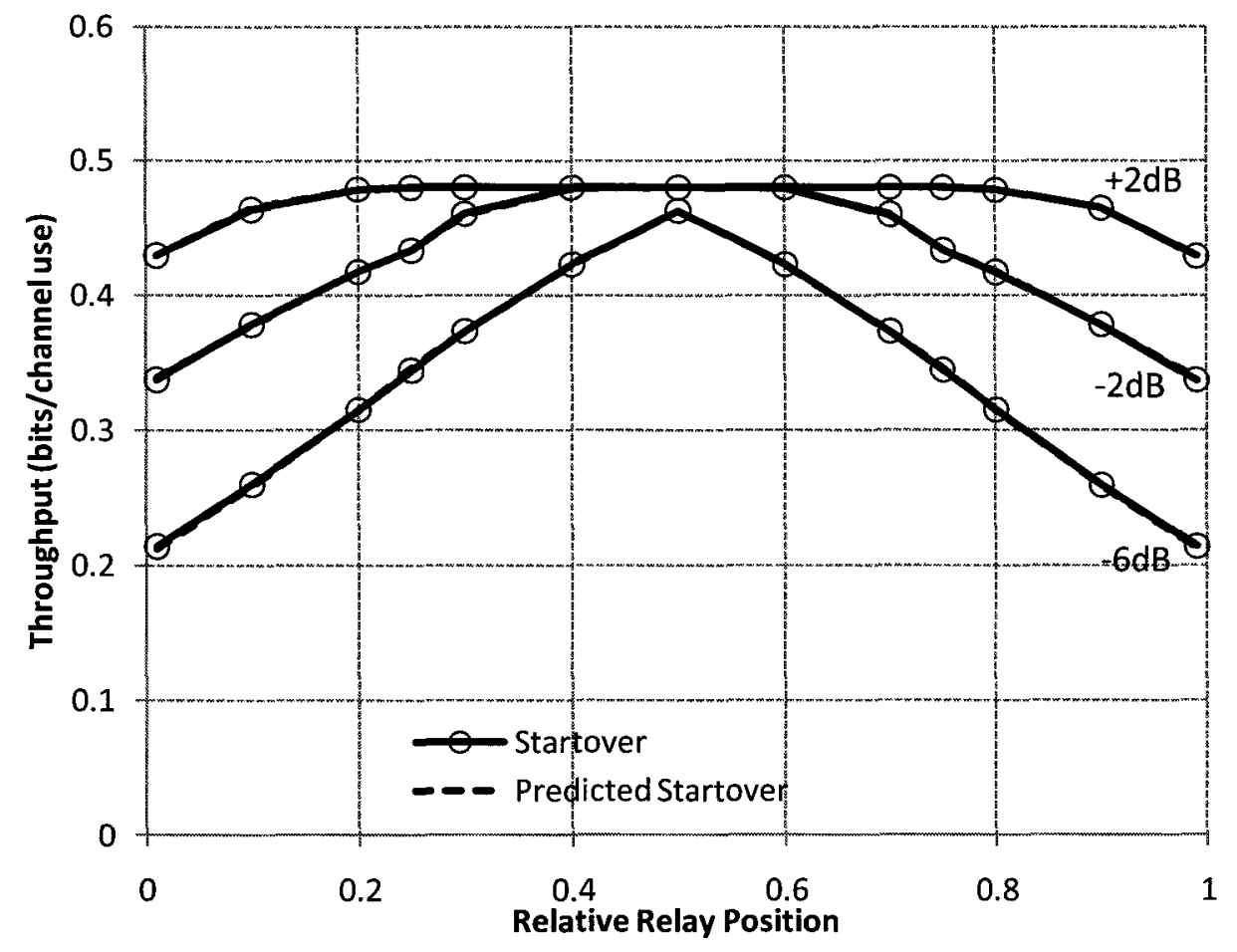

Figure 3.1: Theoretical throughput performance of startover relaying vs. actual throughput performance ( $K=1536$, BPSK).

The comparison of the actual throughput performance of the combine relaying mode with its theoretical prediction (given by Equation (2.43)) at different S-D SNRs, $K=1536$ and BPSK, is depicted in Figures 3.2 to 3.4 .

These figures show an approximate matching between the actual and the theoretically predicted throughput performance for the combine relaying. At $\gamma_{S D}=-6$ $d B$, the actual combine relaying throughout performs worse than the theoretical throughput by 0.05 bit per channel use for the first half distance and then follows closely the theoretical prediction. As the $\gamma_{S D}$ increases, the actual combine throughput follows the predicted throughput more closely. Notice that at $\gamma_{S D}=+2 \mathrm{~dB}$, the actual combine throughput performs little better than expected, by around 0.04 bit per 
channel use and for relay positions that are beyond 0.2 of the distance between the source and the destination.

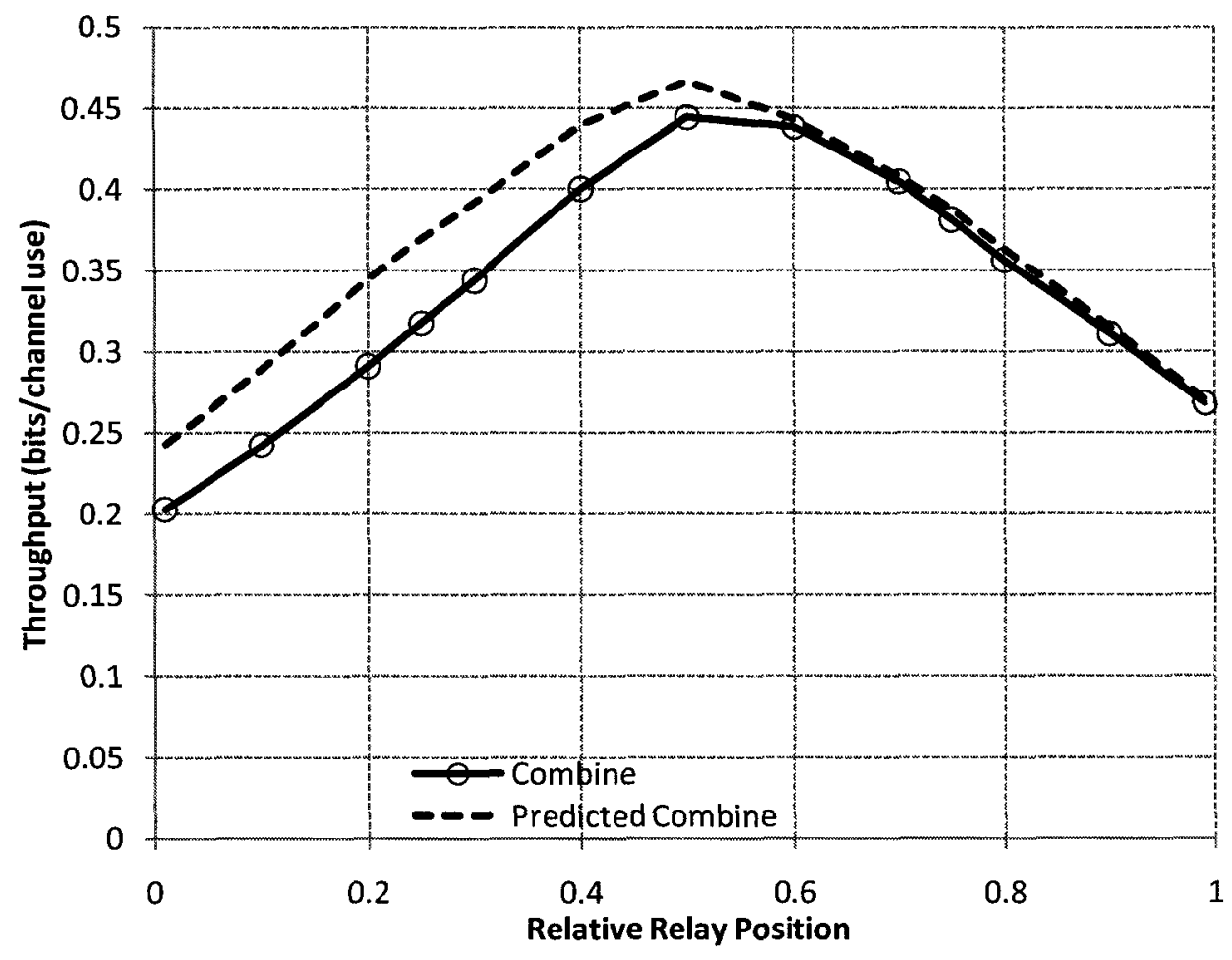

Figure 3.2: Theoretical throughput performance of combine relaying vs. actual throughput performance $\left(K=1536, \mathrm{BPSK}, \gamma_{S D}=-6 \mathrm{~dB}\right)$. 


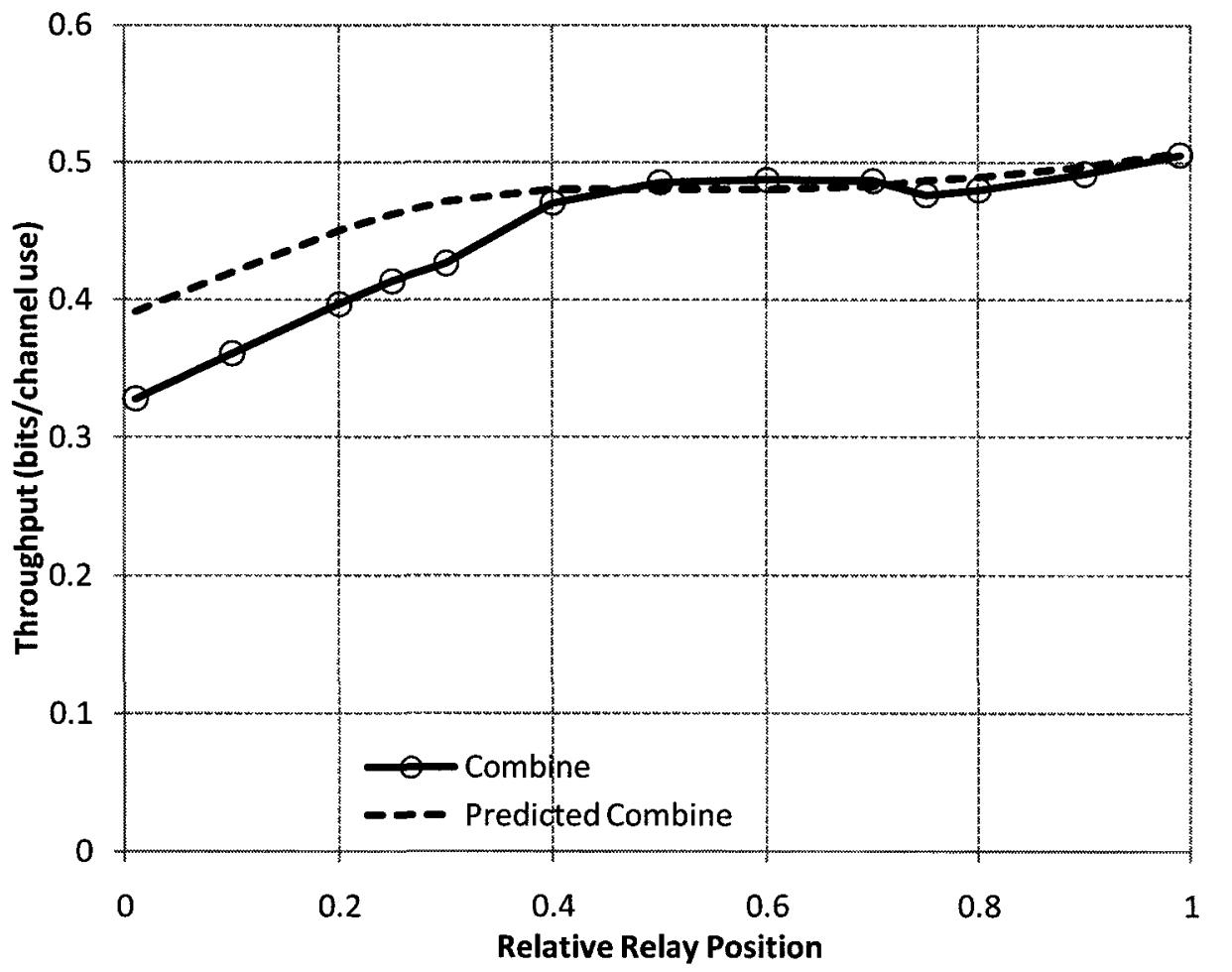

Figure 3.3: Theoretical throughput performance of combine relaying vs. actual throughput performance $\left(K=1536, B P S K, \gamma_{S D}=-2 d B\right)$.

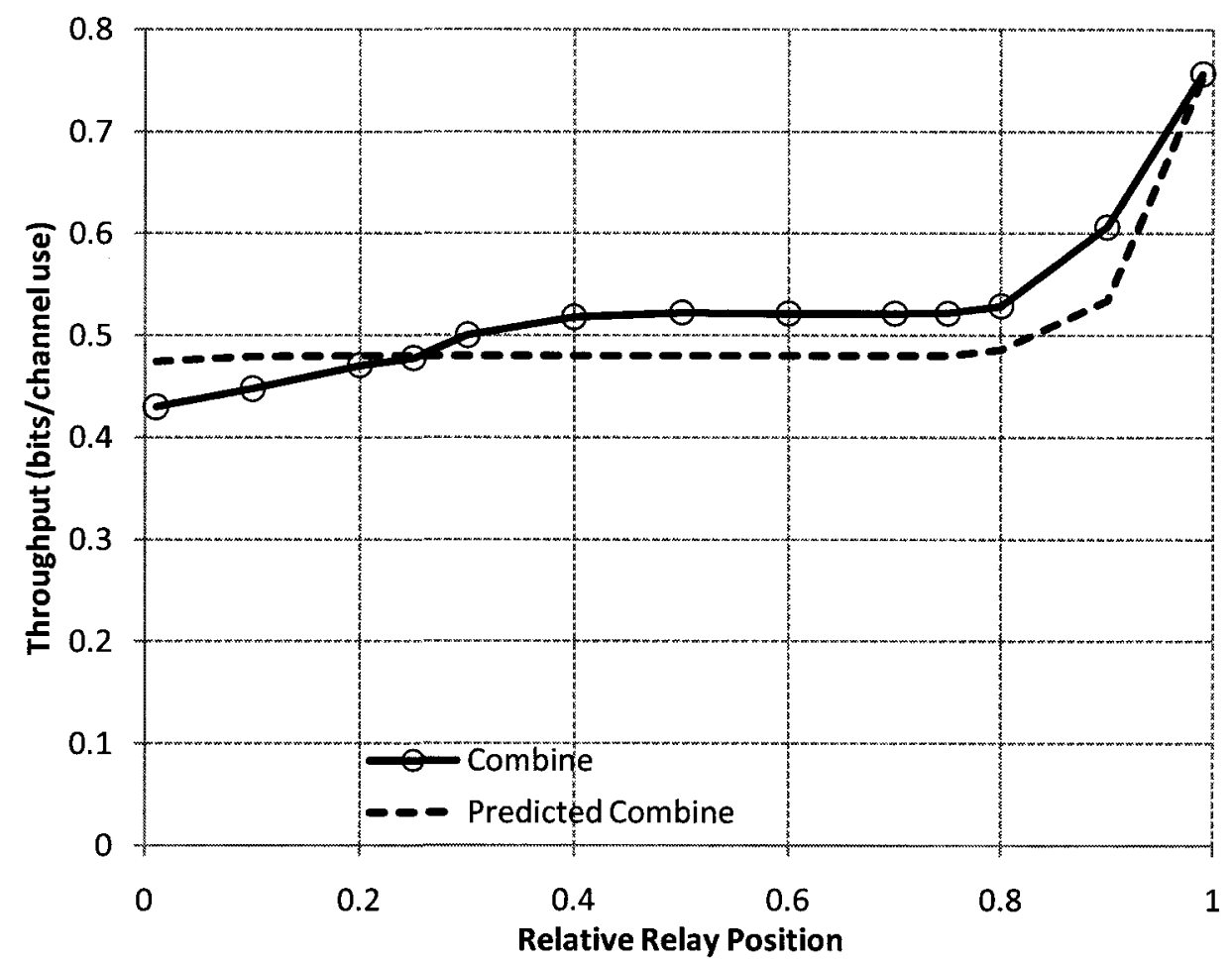

Figure 3.4: Theoretical throughput performance of combine relaying vs. actual throughput performance ( $K=1536$, BPSK, $\gamma_{S D}=+2 \mathrm{~dB}$ ). 
The reason behind these errors in matching the actual with the theoretical combine relaying throughput performance, especially for relay positions near the source, is likely caused by the bad structure of the rateless code used (3GPP RC/QC-LDPC code). As a matter of fact, we already have seen one drawback of this code; that this code fails to reach the $100 \%$ channel throughput at high SNRs. It is evident that the first unpunctured $K$ code bits carry less information than later code bits. Another obvious drawback of this code structure is the uneven distribution of information carried by code bits. This is seen by noticing the unsmooth throughput curve over single-hop transmission. Here we discover another drawback of the code structure; when the code is used for combining samples over low SNRs, it exhibit worse performance than expected. This opens the door for a new research to optimize RC/QC-LDPC codes for combining independent channel samples.

These figures show that the combine relaying throughput still improves as the S-D SNR increases. The maximum throughput for low S-D SNRs tends to be around the mid distance between the source and the destination. However for higher SNRs, the maximum-throughput relay position is closer to the destination.

Figure 3.5 compares the theoretical throughput performance (given by Equation (2.33)) with the actual ones for the continue relaying ( $K=1536$ and BPSK) over different S-D SNRs. This figure shows the close matching of the theoretical to the actual throughput performance. The continue relaying mode follows closely the theoretically predicted model because it does not repeat code bits; it always uses new code bits. The little differences between the theoretical and the actual throughputs could be because 
of the unequal amount of information carried by the code bits and the interdependency of the code bits. However overall, the matching here is considered valid. One can dissert that a well structured rateless code (independent code bits, and equal information distribution among code bits) would provide a better match to the theoretical throughput performance.

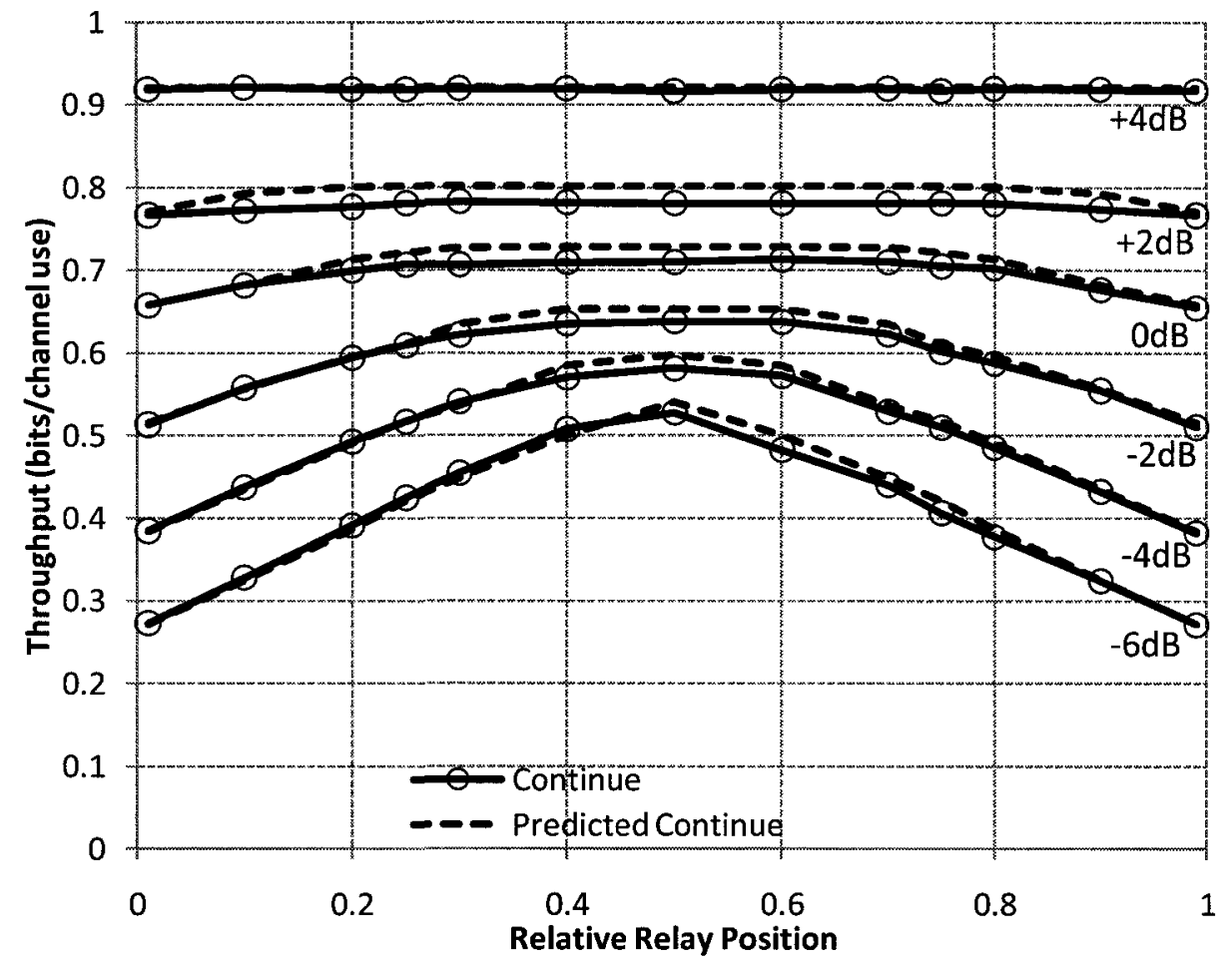

Figure 3.5: Theoretical throughput performance of continue relaying vs. actual throughput performance ( $K=1536$, BPSK).

Notice that continue relaying mode also improves as the S-D SNR increases. For low SNRs, the optimum relay position is at the mid distance between the source and the destination. As the S-D SNR increases, the range of optimum relay positions broadens around the mid distance; similar to the startover relaying and different from the combine relaying. 
Figure 3.6 compares the three relaying modes (startover, combine and continue) with the single-hop transmission, for $K=1536, \mathrm{BPSK}$, and $\gamma_{S D}=-6 \mathrm{~dB}$. At this very low SD SNR, all the three relaying modes surpass the single-hop transmission for a wide range of relay positions. For relay positions which are very close to the source, the throughputs of the startover and the combine relaying modes are less than the singlehop transmission. This is because the R-D SNR is very close to the S-D SNR; moreover, the relaying process requires additional code bit transmissions from the source to the relay. As the relay moves away from the source and gets closer to the midpoint between the source and the destination, the R-D SNR improves while maintaining a good S-R SNR level and consequently improves the decoding speed at the destination and the overall throughput. The maximum throughput for both the startover and the combine relaying modes occur at the midpoint between the source and the destination. It is a one point maximum peak, and as the relay deviates from the midpoint the throughput decreases gradually. As the relay moves closer to the destination and away from the midpoint, the relaying throughput decreases. As the relay position moves even closer to the destination, the startover relaying mode throughput goes below the singlehop transmission because the S-D and S-R SNRs are very close in value and because relaying requires more code bit transmissions than the single-hop. 


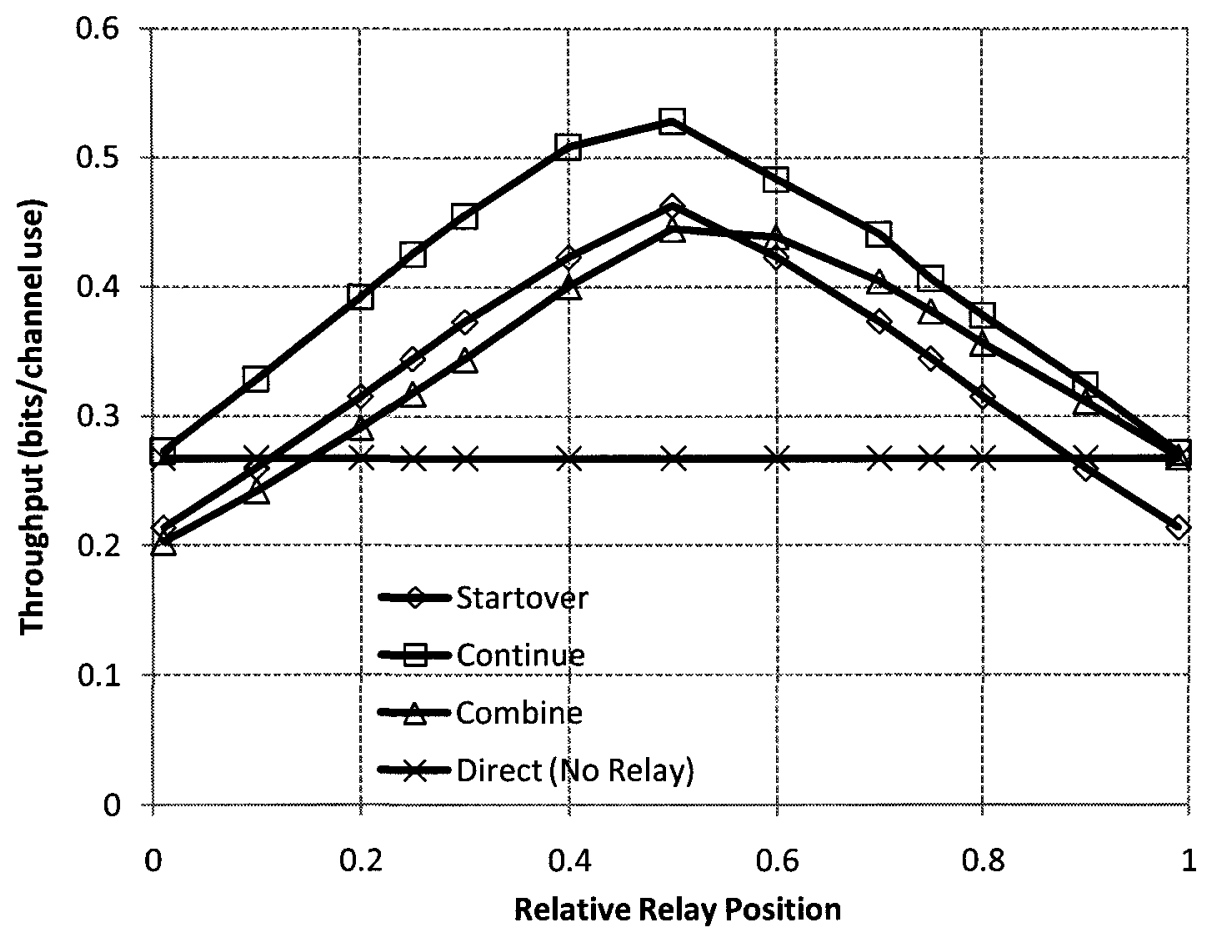

Figure 3.6: Comparison of linear relay performance: $K=1536, B P S K, \gamma_{S D}=-6 \mathrm{~dB}$.

The throughput of startover relaying is a little better than combine relaying for the first half distance and a little worse than combine relaying for almost the second half distance. It is worth mentioning that combining was not expected to enhance the throughput of relaying. Chase predicted that combining doesn't improve the throughput of transmission over SNR, it only enhances the error rate and especially in the case of jamming [25]. The reason why combine relaying performs worse than startover relaying for the first half of the distance between the source and the destination is mainly because of the bad code structure with combining. Also, repeated code bits carry less information and their amount in the decoded codeword is more when the relay is closer to the source. However, when the relay is closer to the destination, the relay takes more code bits to decode the codeword, making the destination receive more code bits other than from the first part of the codeword. Combine relaying utilizes that feature 
while startover does not. Overall, at low SNRs combine and startover relaying perform similarly.

The continue relaying outperforms the single-hop and other relaying methods. This is because the destination better utilizes the directly transmitted bits, it does not use repeated bits, and the extra transmitted bits by the relay are always new code bits. In fact, continue relaying complements the direct single-hop transmission. As so, its throughput is never below the single-hop throughput. Notice that with continue relaying, the destination decoder avoids combining code bits which may cause strange behavior (as the case with combine relaying)..

When the relay is very close to the source, the relay decodes the codewords faster. But because the S-D and R-D SNRs are very close in value, the continue relaying throughput is close to the single-hop transmission throughput. As the relay gets closer to the midpoint, the destination receives better quality code bits, improving the relaying throughput. The maximum through occurs exactly at the midpoint. As the relay moves further towards the destination, the S-R SNR weakens more and the R-D SNR improves. This delays the decoding process at the relay and hence degrades the throughput of relaying. The continue relaying throughput is symmetric around the midpoint.

Figures 3.7 to 3.11 show the throughout performances of the three relaying modes for $K=1536$ and BPSK over higher S-D SNRs. The startover relaying throughput improves as the S-D SNR increases. Also, the maximum throughput range broadens around the midpoint. The maximum throughput saturates at 0.48 bits/channel use, even for those cases where the direct single-hop transmission exceeds that value. It is clear that the 
startover throughput is always symmetric around the midpoint distance between the source and the destination.

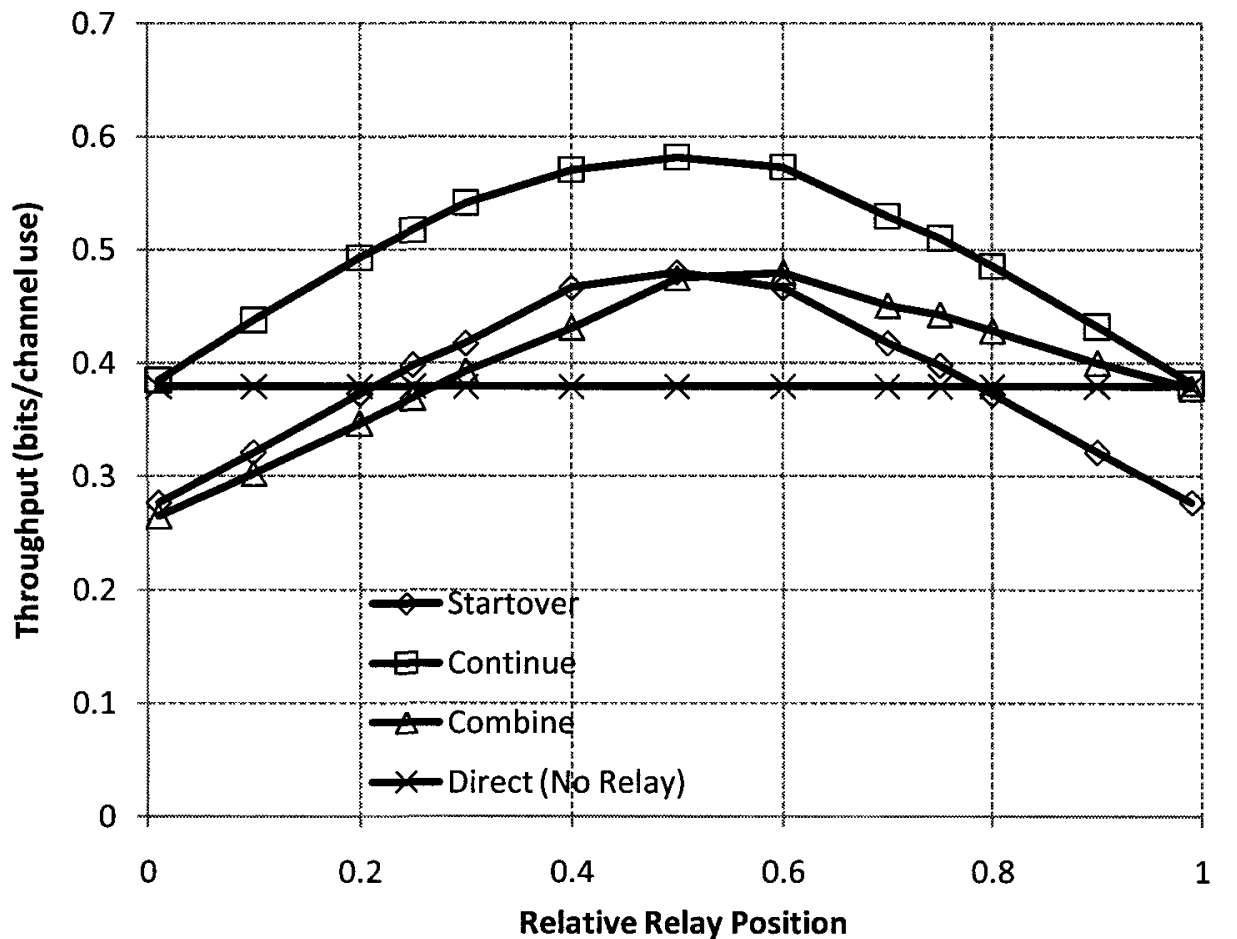

Figure 3.7: Comparison of linear relay performance: $K=1536, B P S K, \gamma_{S D}=-4 \mathrm{~dB}$.

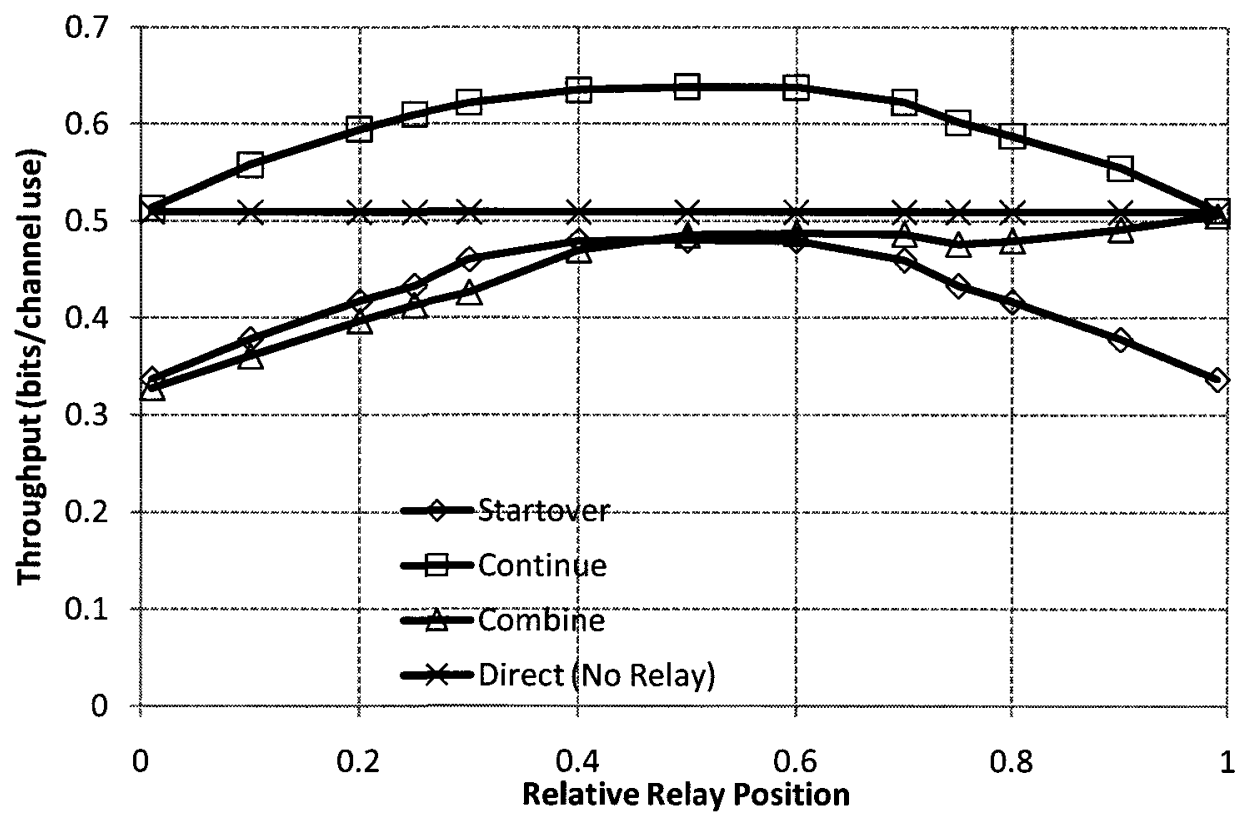

Figure 3.8: Comparison of linear relay performance: $K=1536, B P S K, \gamma_{S D}=-2 d B$. 


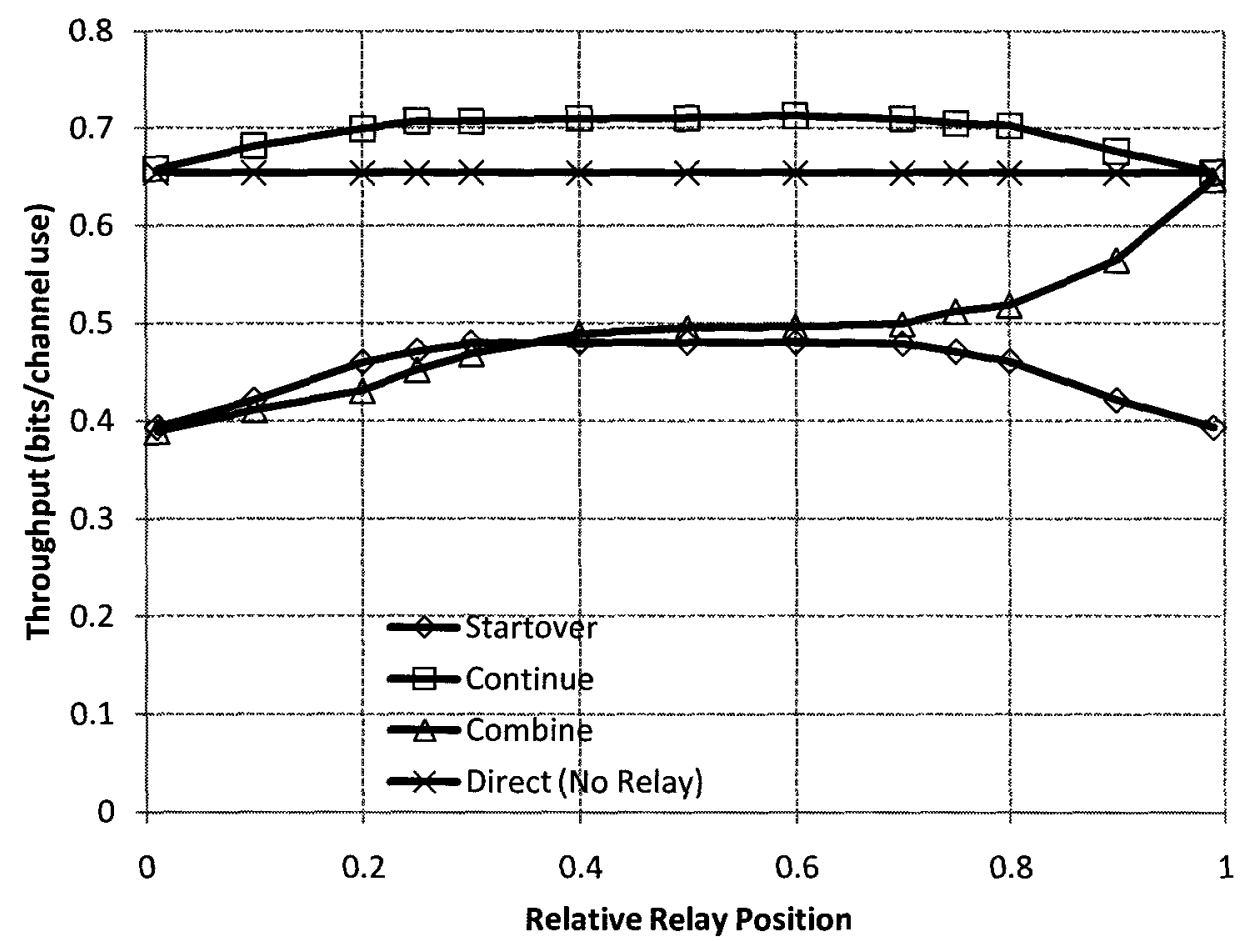

Figure 3.9: Comparison of linear relay performance: $K=1536, B P S K, \gamma_{S D}=0 \mathrm{~dB}$.

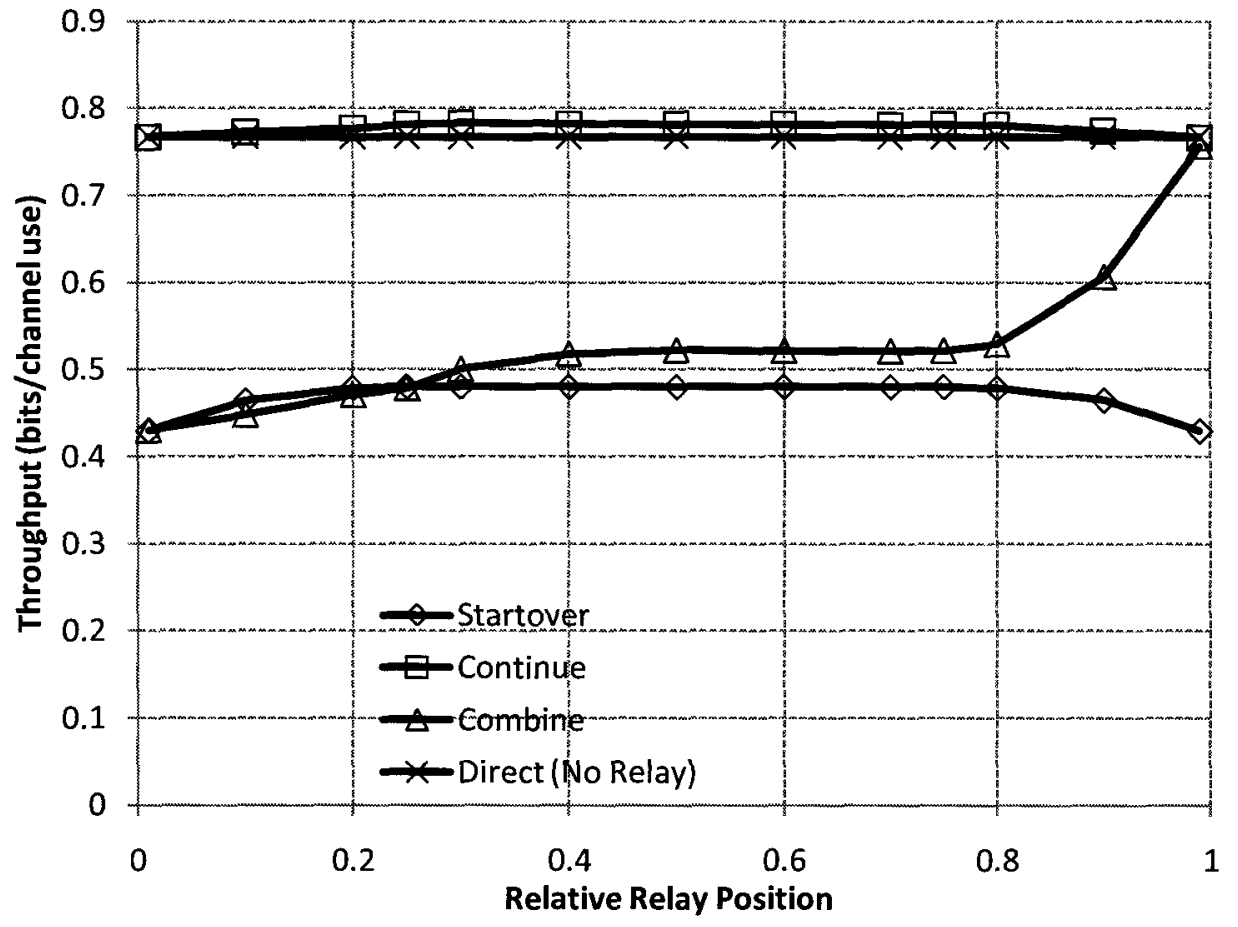

Figure 3.10: Comparison of linear relay performance: $K=1536, B P S K, \gamma_{S D}=+2 d B$. 


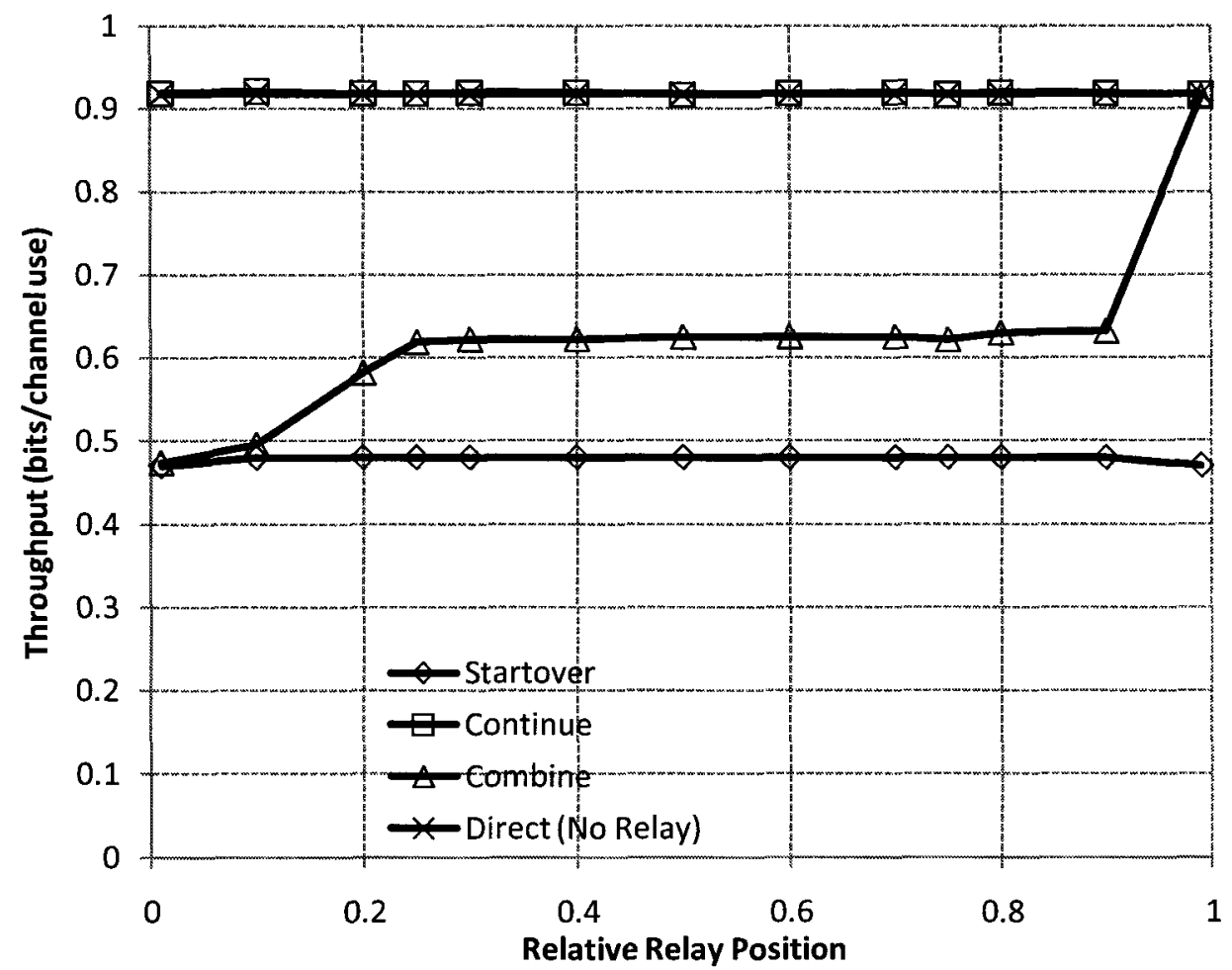

Figure 3.11: Comparison of linear relay performance: $K=1536, B P S K, \gamma_{S D}=+4 \mathrm{~dB}$.

When the direct transmission throughput is below the maximum startover throughout ( $0.48 \mathrm{bits} / \mathrm{channel}$ use), the combine relaying throughput performs worse than the startover for the first half distance which is closer to the source and performs better than the startover relaying for the other half. For higher S-D SNR where the single-hop transmission throughout is better than the maximum startover throughput, the range over which the combine relaying outperforms the startover relaying mode broadens toward the source and eventually for higher SNRs the combine relaying totally outperforms the startover relaying throughput. Unfortunately, this improvement does not beat the single-hop transmission at those high S-D SNRs.

As for the continue relaying mode, the throughput improves as the S-D SNR increases and for higher SNRs the throughput saturates to the single-hop transmission 
throughput. In other words, the continue relaying throughout is always better than the single-hop relaying throughput for low and medium S-D SNR levels, and equal to it for higher S-D SNR levels. The continue relaying is self adjusted and capable of improving the transmission throughout whenever possible. It is always no worse than the other techniques, and is often much better.

\subsubsection{Simulation Results for $K=\mathbf{3 8 4}$ and BPSK}

This section shows the simulation results of relaying for BPSK and a short codeword length of $K=384$. Figures 3.12 to 3.16 compare the actual throughput for the three relaying modes with their theoretical values. The results are similar to the long codeword results; the actual and the theoretical throughputs for the startover relaying match exactly, the actual and the theoretical throughputs of the combine relaying approximately match (at least in behavior), and the actual and the theoretical throughputs of the continue relaying match closely. The theoretical and actual relaying throughout match better than for long codewords except at very low S-D SNR levels (-6 dB), while for high SNR levels the long codewords match better. The reason is not clear. Over all, the theoretical prediction for both startover and continue relaying is considered valid, but approximate for combine relaying. 


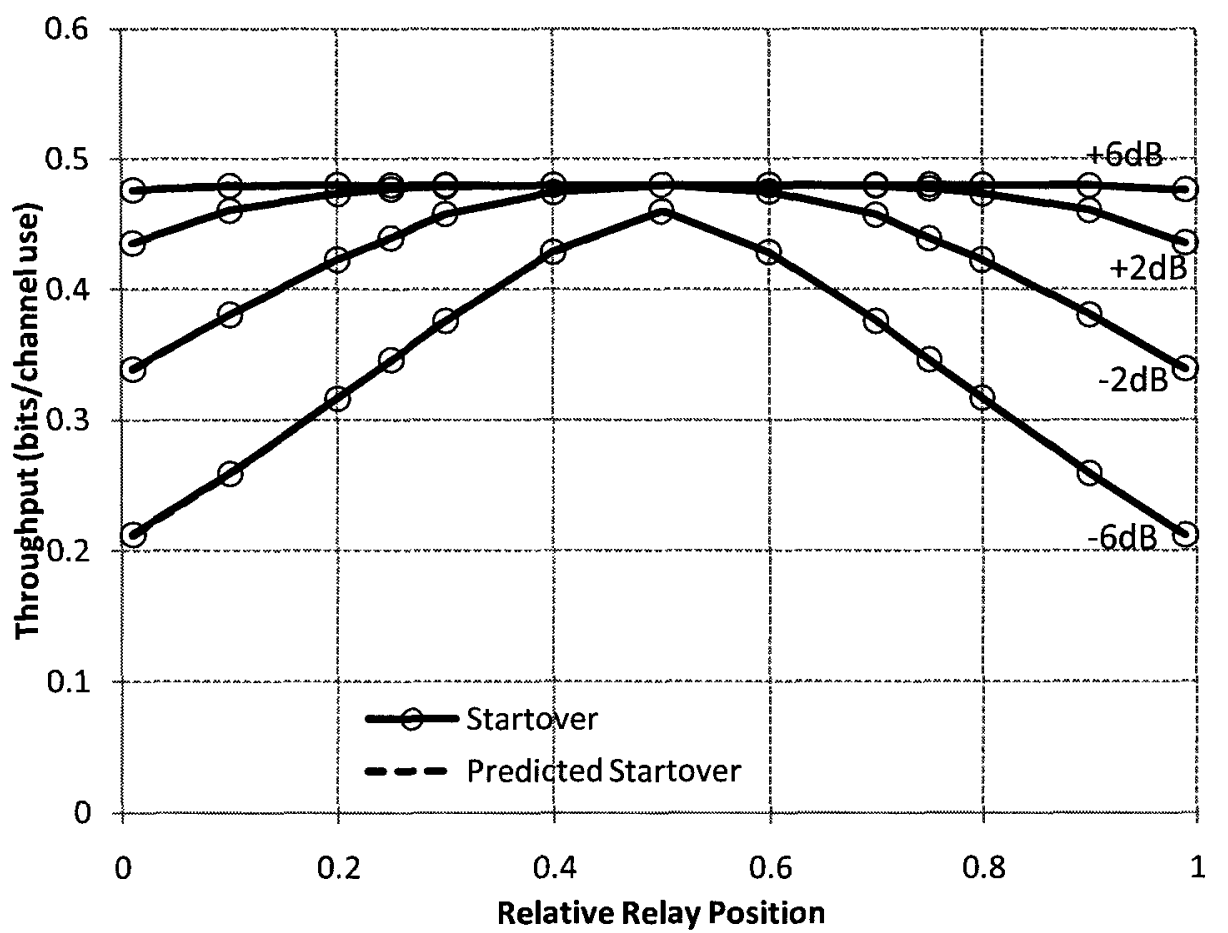

Figure 3.12: Theoretical throughput performance of startover relaying vs. actual throughput performance ( $K=384$, BPSK).

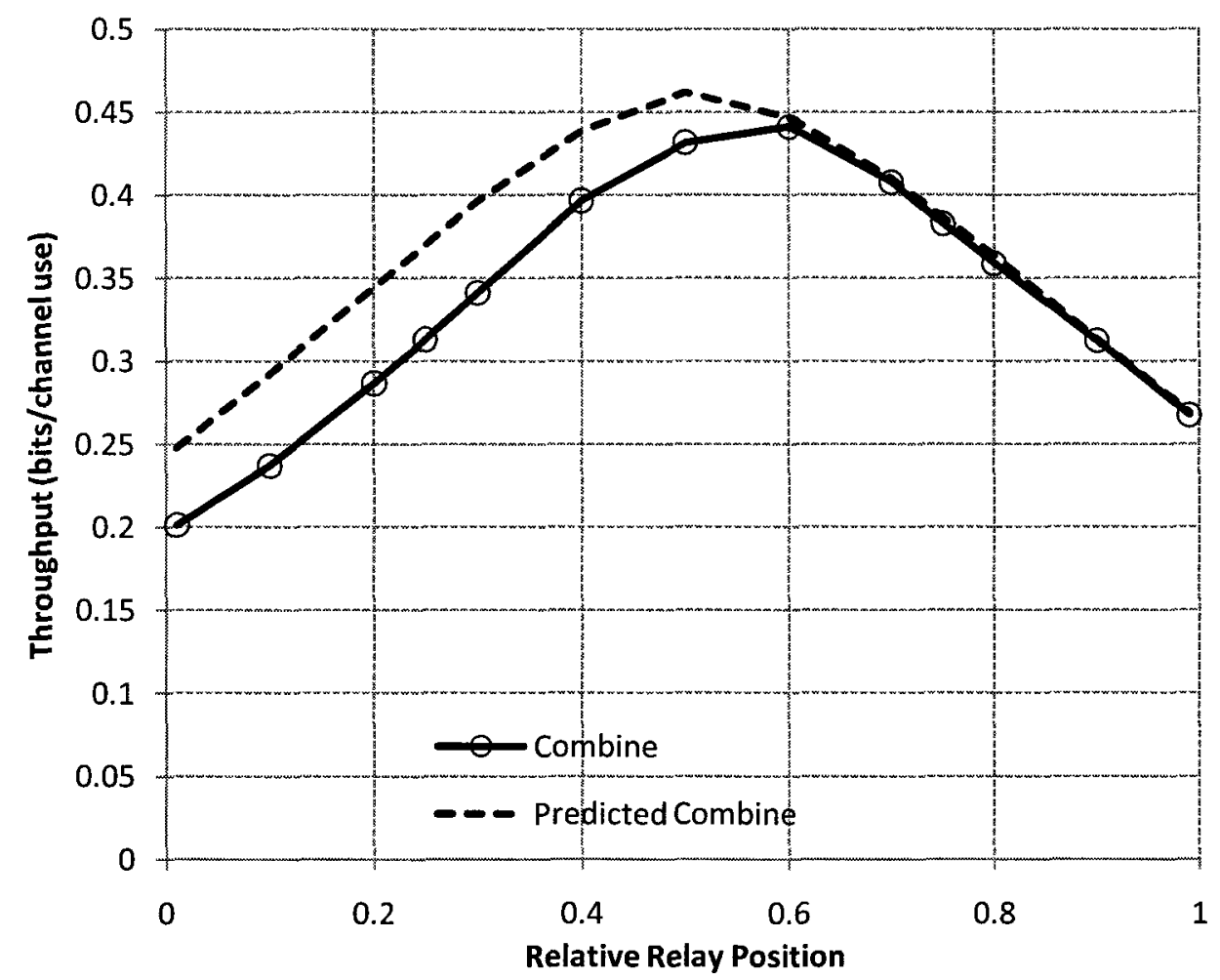

Figure 3.13: Theoretical throughput performance of combine relaying vs. actual throughput performance $\left(K=384\right.$, BPSK, $\left.\gamma_{S D}=-6 \mathrm{~dB}\right)$. 


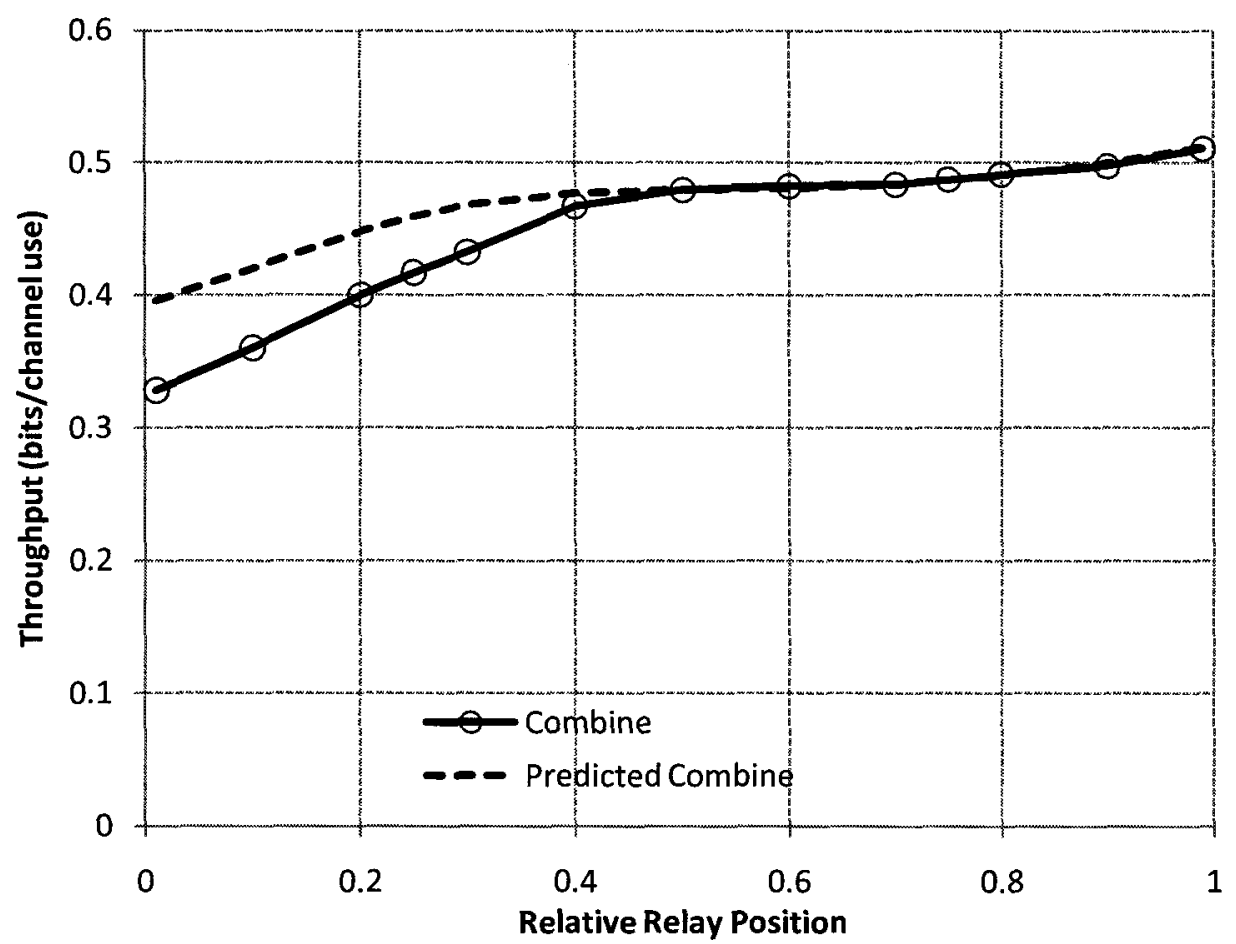

Figure 3.14: Theoretical throughput performance of combine relaying vs. actual throughput performance $\left(K=384, B P S K, Y_{S D}=-2 \mathrm{~dB}\right)$.

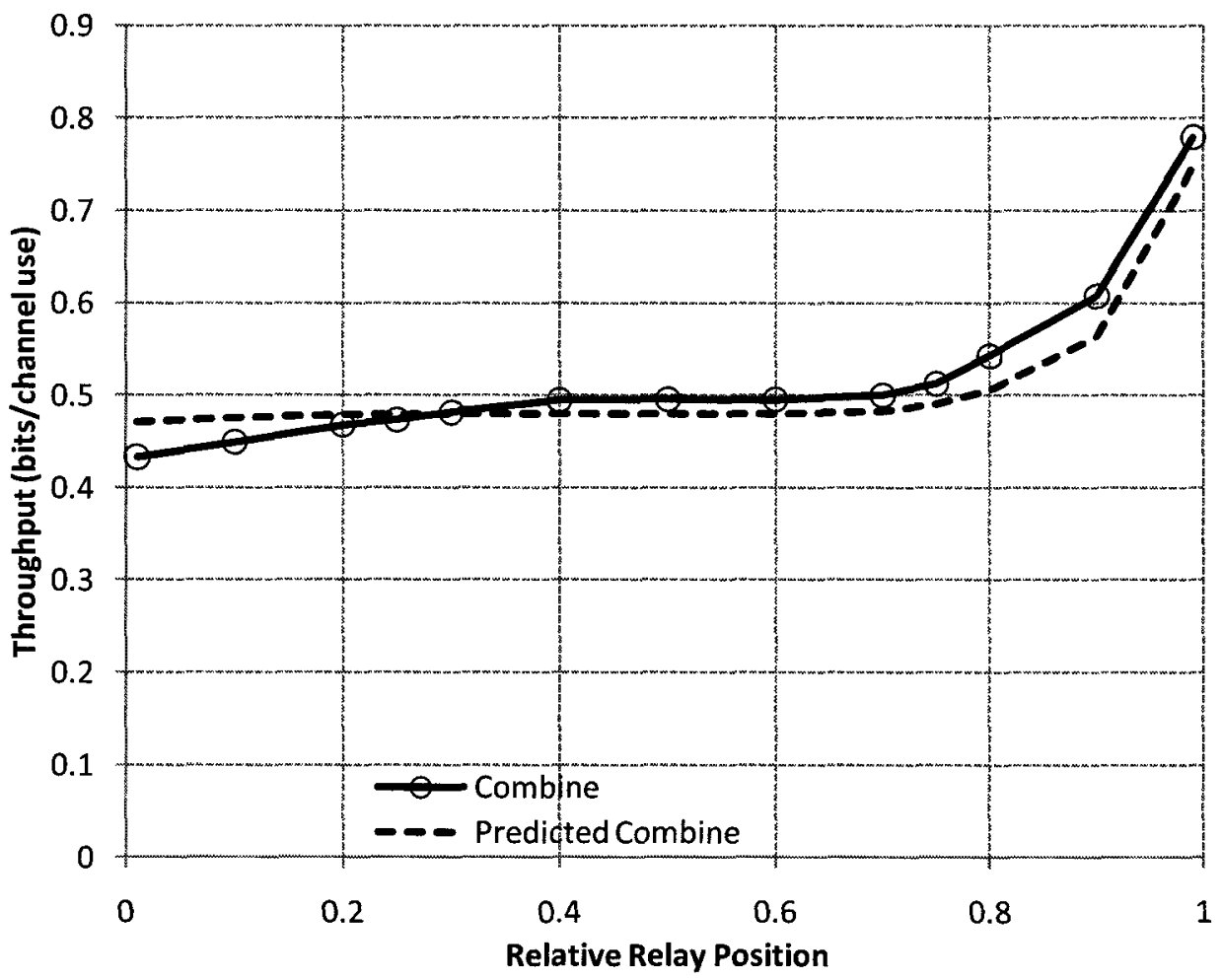

Figure 3.15: Theoretical throughput performance of combine relaying vs. actual throughput performance ( $K=384, B P S K, \gamma_{S D}=+2 \mathrm{~dB}$ ). 


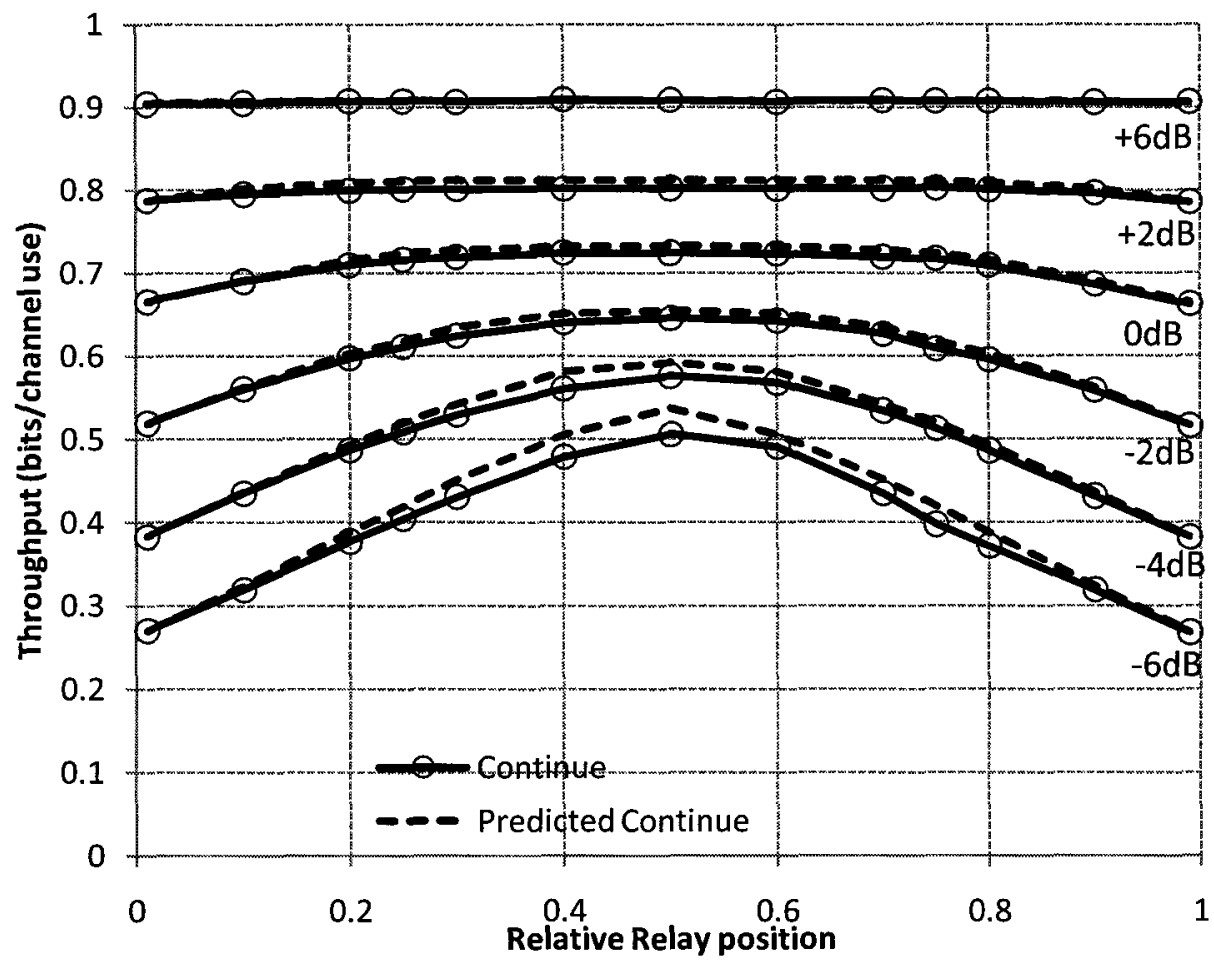

Figure 3.16: Theoretical throughput performance of continue relaying vs. actual throughput performance ( $K=384$, BPSK).

Figures 3.17 to 3.23 depict the comparison of the actual throughput performances of the three relaying modes and the single-hop. The overall throughput of relaying improves as the S-D SNR increases, while continue relaying always beat the other relaying modes and the single-hop relaying. Combine and startover relaying outperform the single-hop throughout for low SNRs and perform worse for high SNRs. The results of $K=384$ BPSK are very similar to $K=1536$ BPSK. This reveals that the relaying mode relative performance is independent of the message size.

The curves here are smoother than the ones of the long codeword lengths $(K=$ 1536). It is mainly because of the code structure itself; the single-hop throughput for $K=$ 384 is smoother than $K=1536$. 


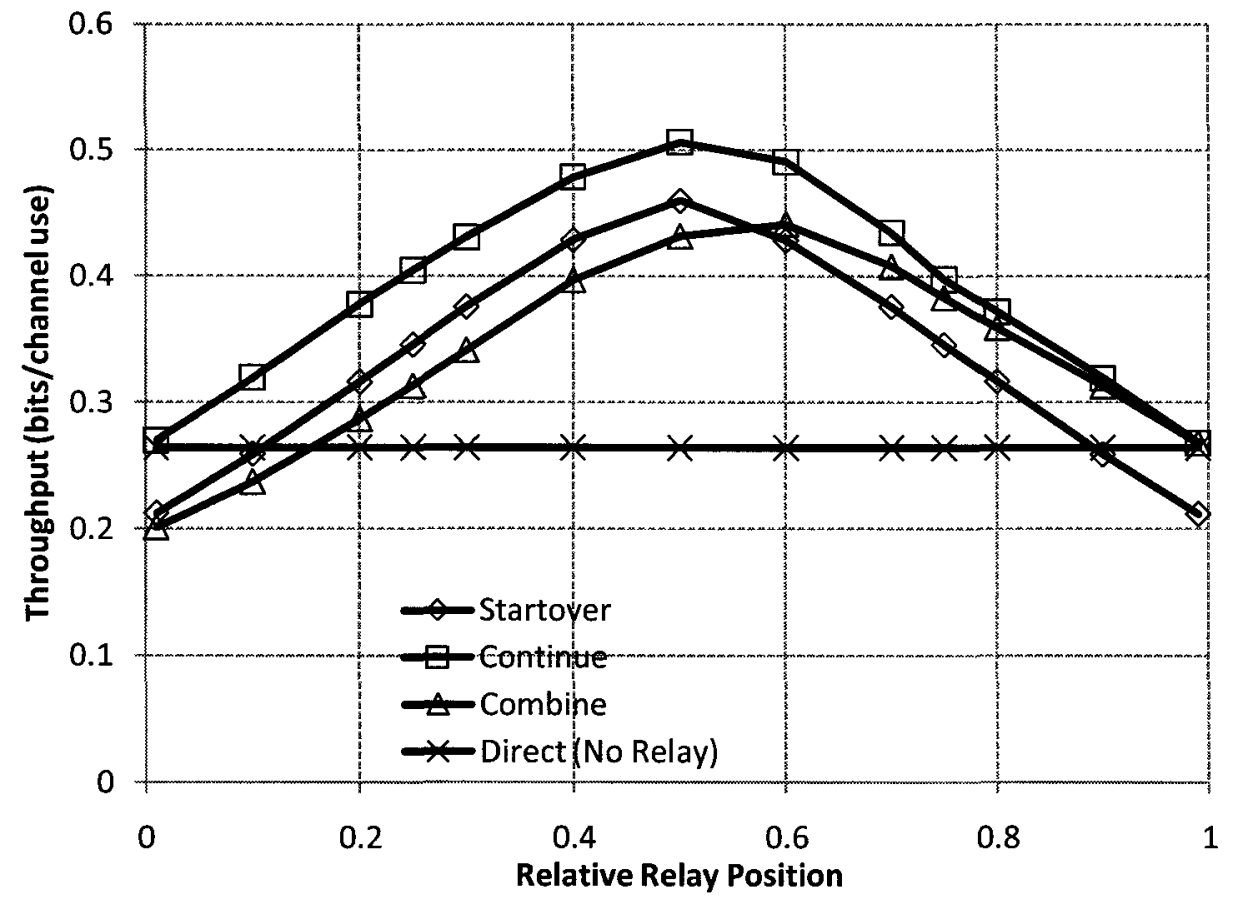

Figure 3.17: Comparison of linear relay performance: $K=384$, BPSK, $\gamma_{S D}=-6 \mathrm{~dB}$.

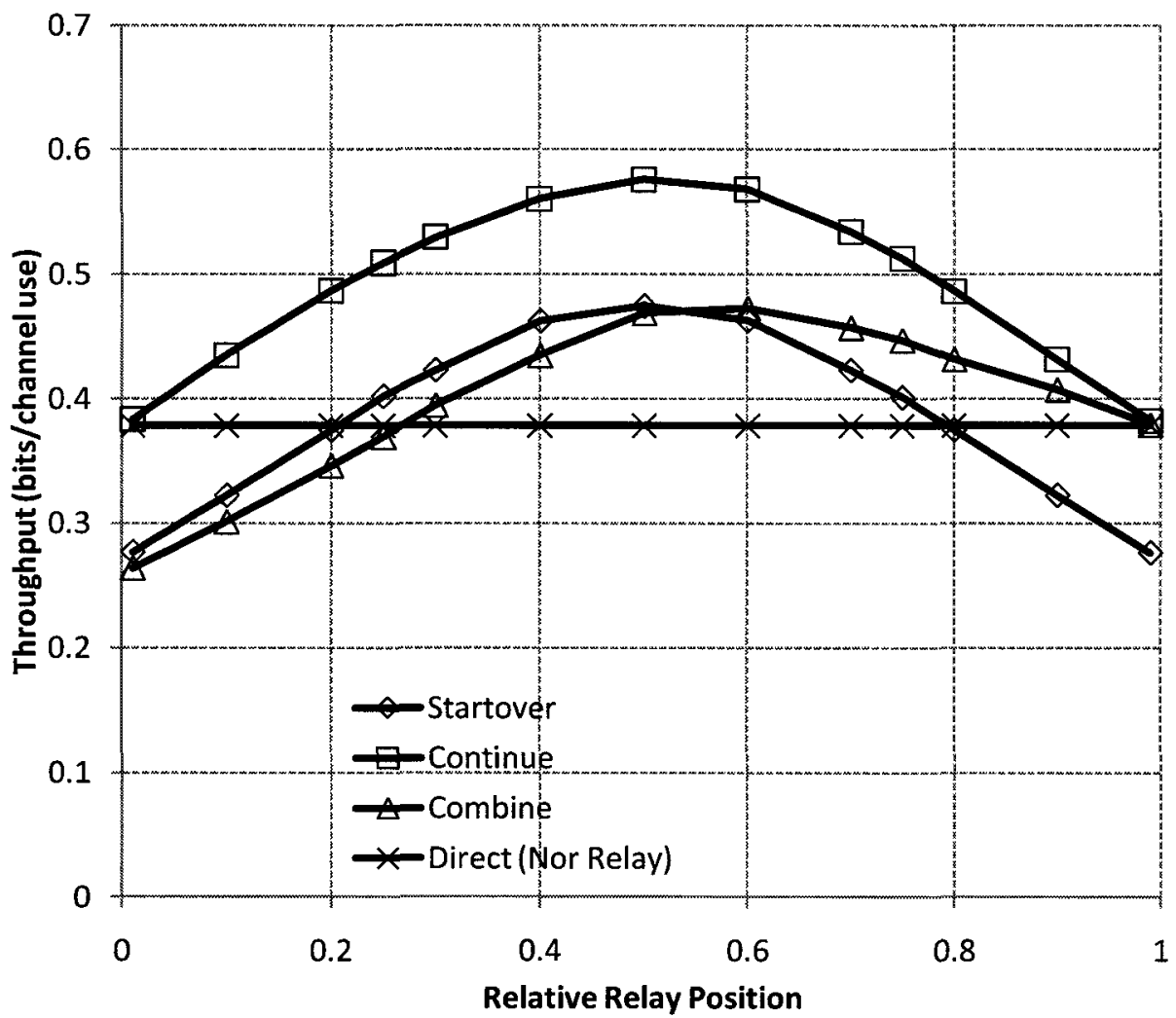

Figure 3.18: Comparison of linear relay performance: $K=384$, BPSK, $\gamma_{S D}=-4 \mathrm{~dB}$. 


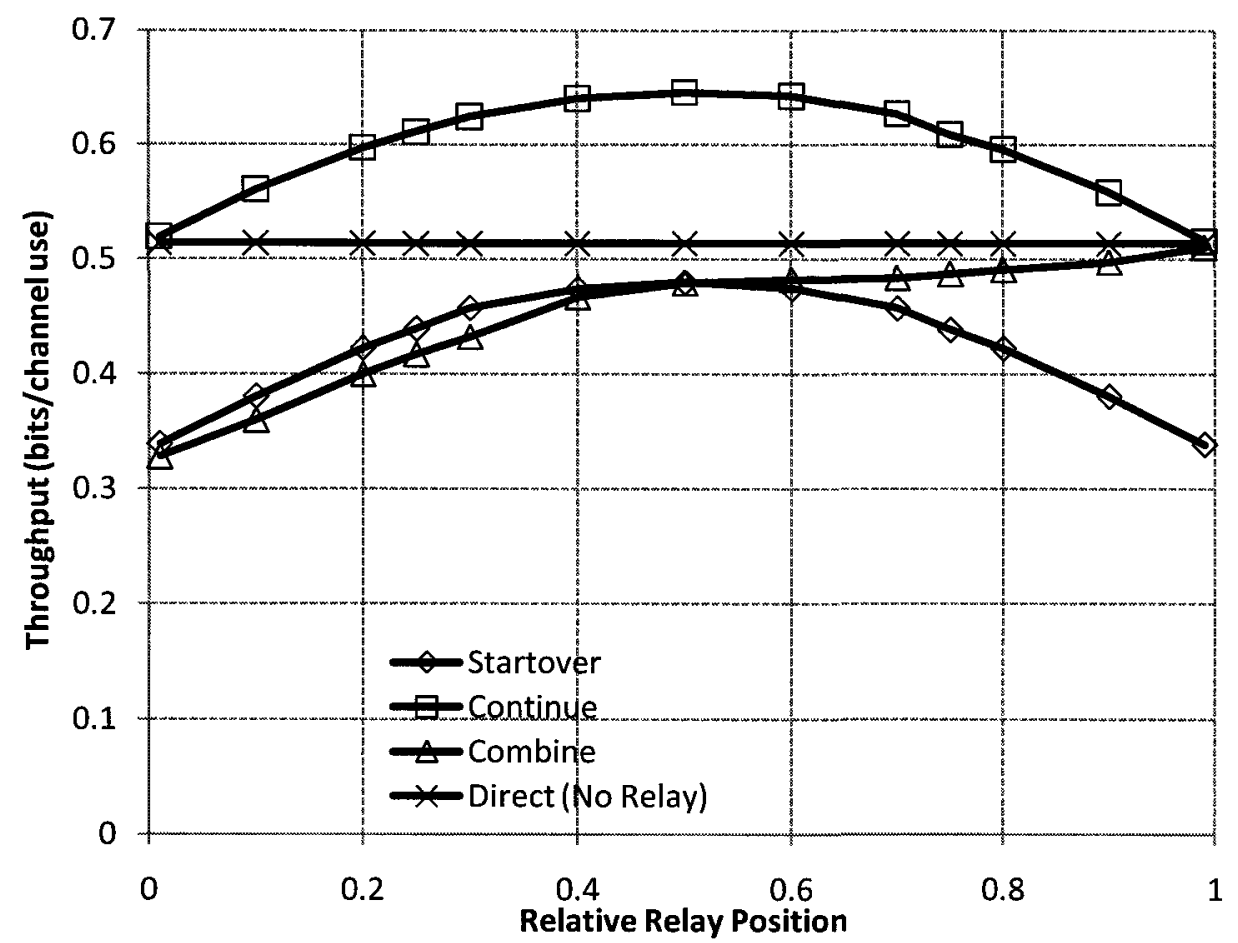

Figure 3.19: Comparison of linear relay performance: $K=384, B P S K, \gamma_{S D}==-2 \mathrm{~dB}$.

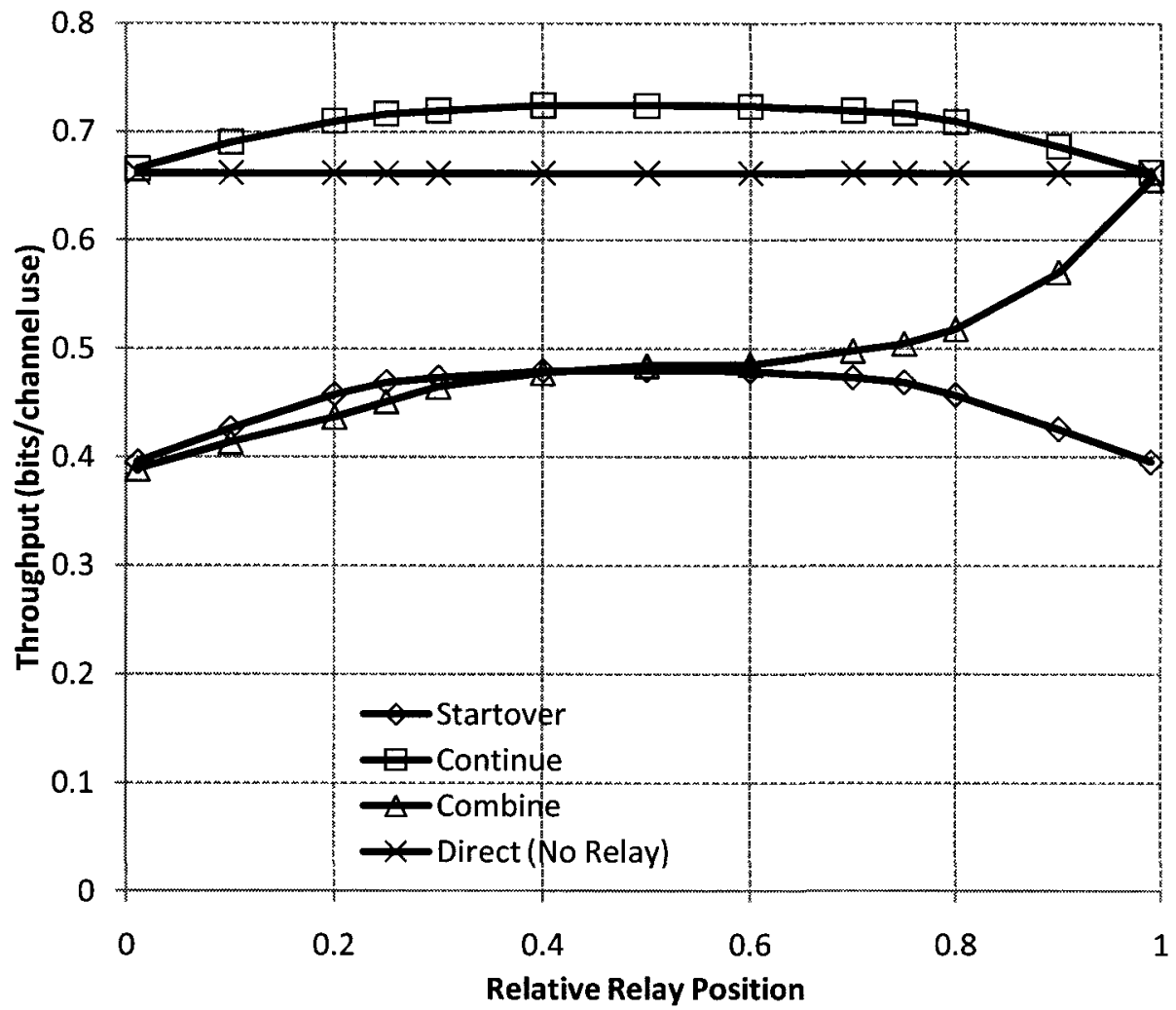

Figure 3.20: Comparison of linear relay performance: $K=384, B P S K, \gamma_{S D}=0 \mathrm{~dB}$. 


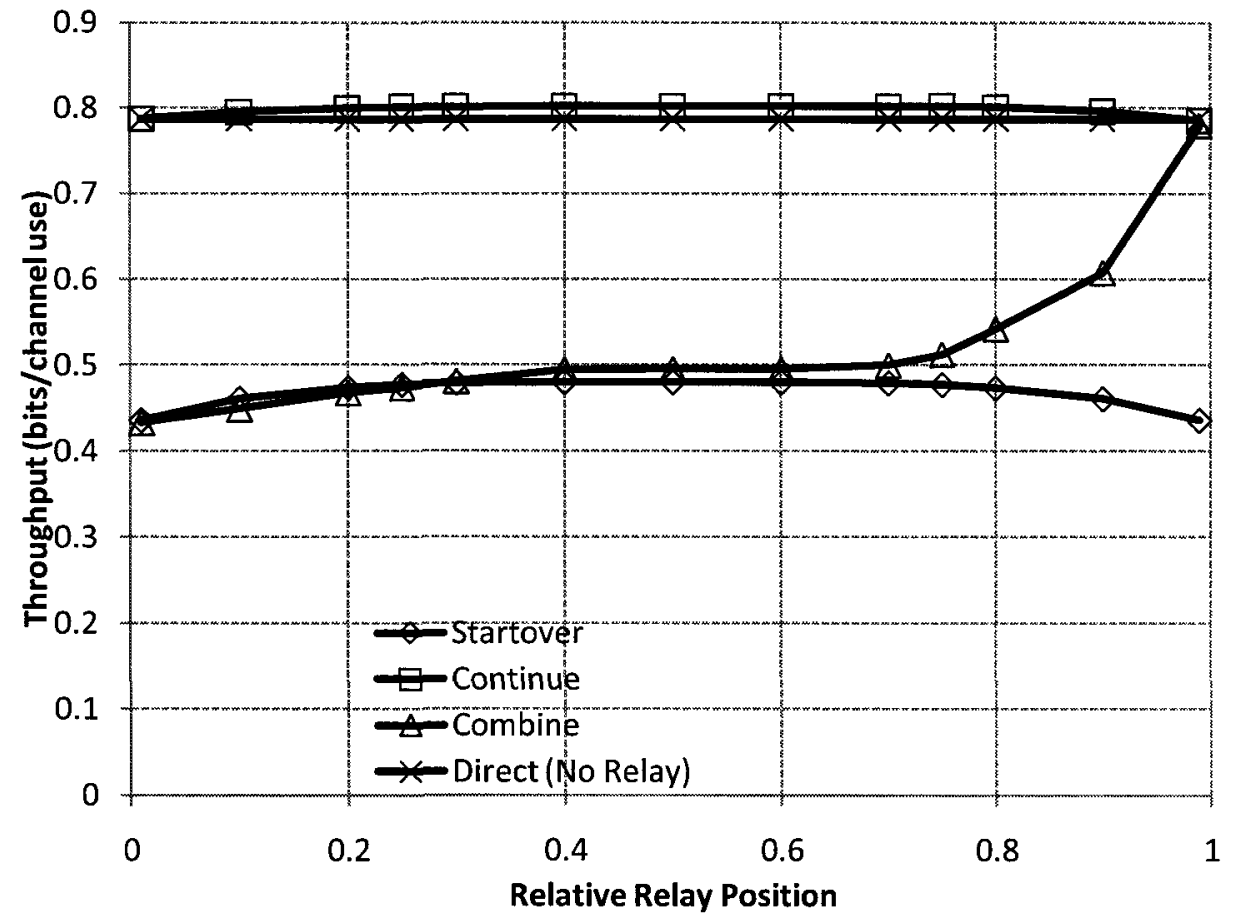

Figure 3.21: Comparison of linear relay performance: $K=384, B P S K, Y_{S D}=+2 \mathrm{~dB}$.

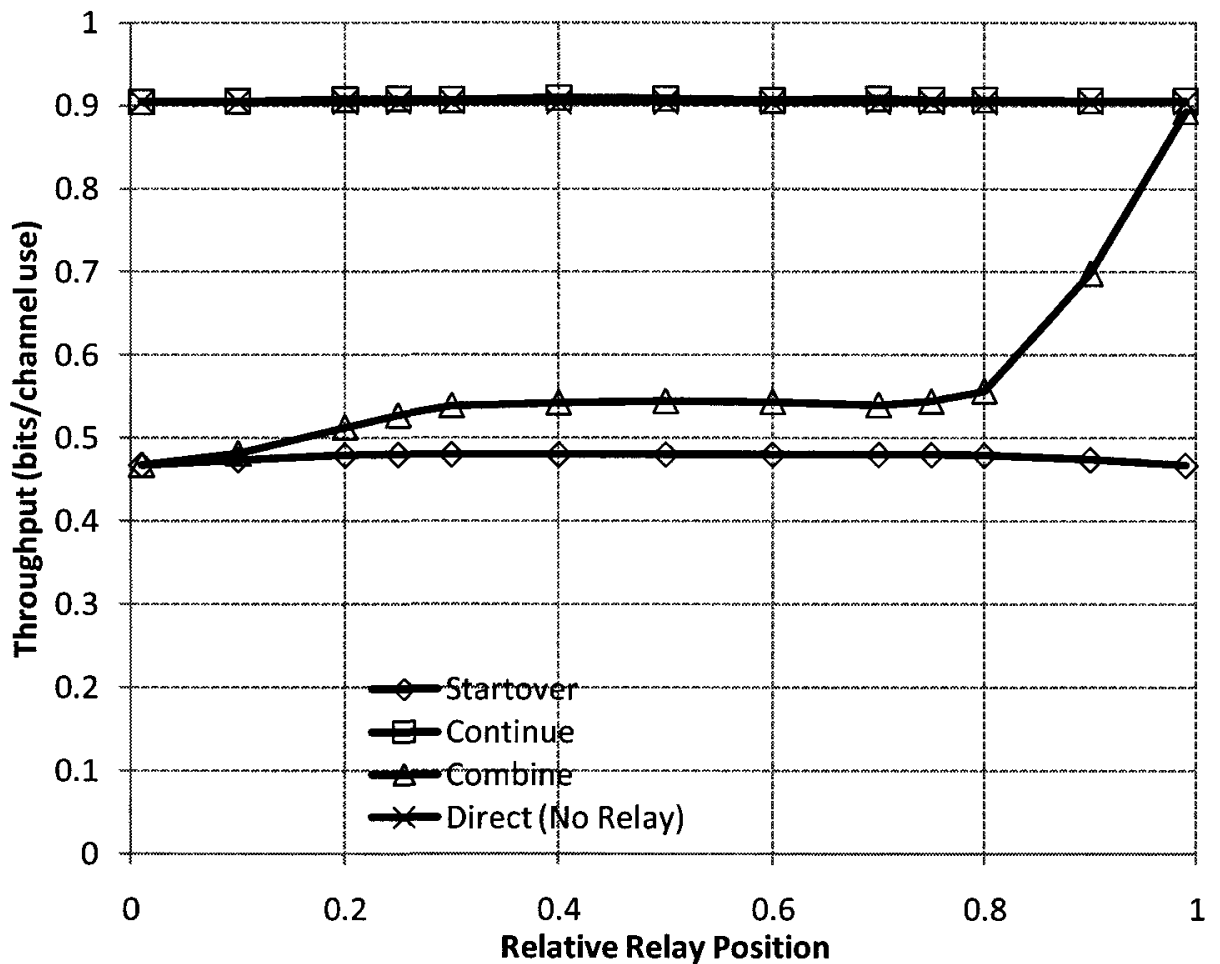

Figure 3.22: Comparison of linear relay performance: $K=384, B P S K, \gamma_{S D}=+4 \mathrm{~dB}$. 


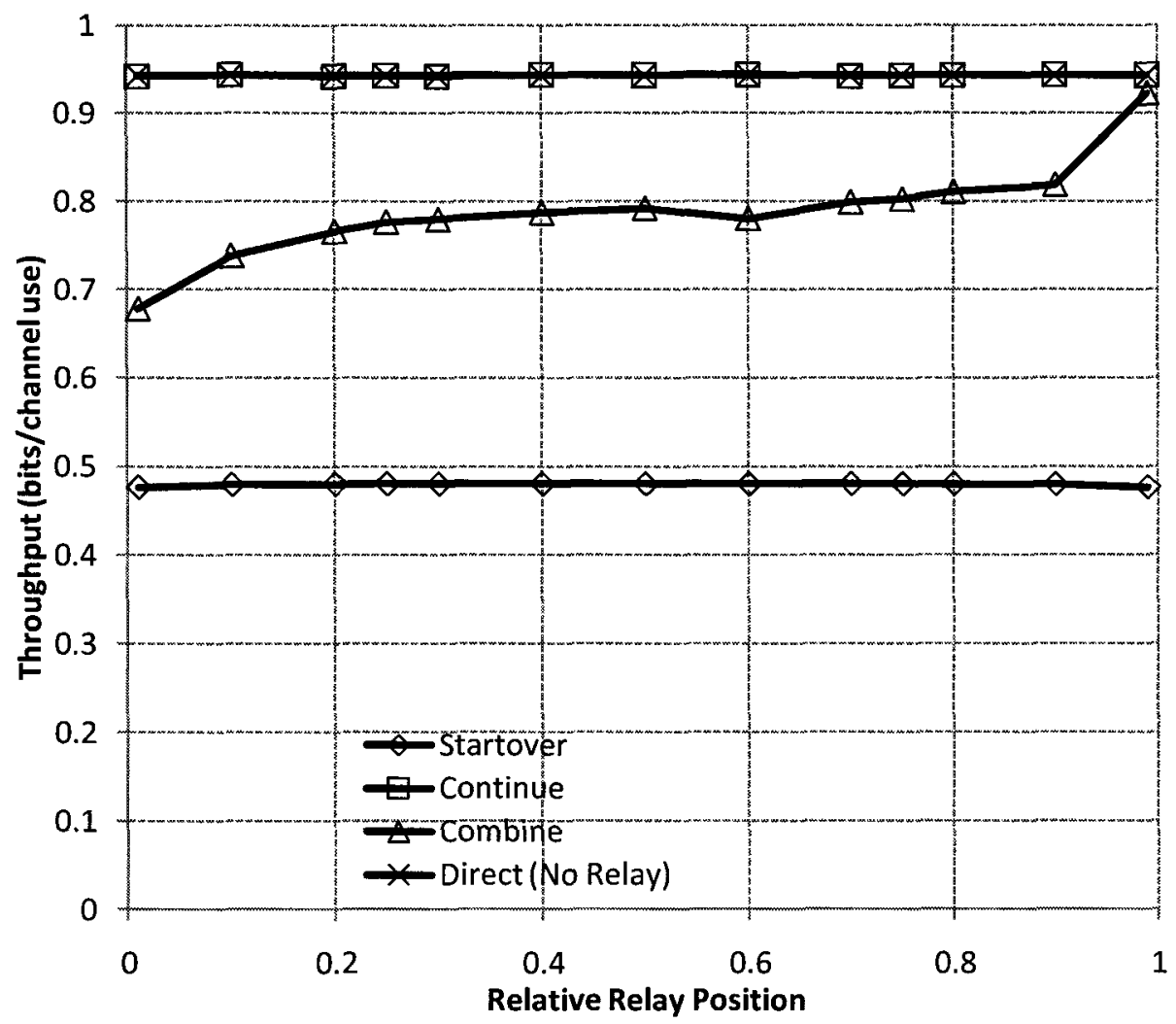

Figure 3.23: Comparison of linear relay performance: $K=384, B P S K, Y_{S D}=+6 \mathrm{~dB}$.

\subsubsection{Simulation Results for $K=384$ and 16-QAM}

This section presents the simulation results for the startover relaying mode and continue relaying mode using 16-QAM modulation. It was shown in the previous two sections that the combine relaying mode does not match exactly the theoretical performance, and performs badly in general in terms of throughput. As so, the combine relaying is discarded from further simulations. The simulations here uses codewords of length $K=384$ since it is easier and faster to simulate than codewords of $K=1536$, and as seen before the relative throughput performance of the relaying mode is independent from the codeword size.

Figure 3.24 shows the comparison of the actual and the theoretical throughput of the startover relaying for 16-QAM modulation and $K=384$. It is clear that the matching 
between the two curves is perfect. The throughput increases as the S-D SNR increases, and saturates at the value 1.92 bits/channel use. This value is predicted by the startover relaying mode throughput formula (2.28); when S-R and R-D are in their best conditions with throughput of 3.84 bits/channel use. The maximum occur when the relay is located at the midpoint between the source and the destination. This maximum value increases as the S-D SNR level increases and saturates at 1.92 bits/channel use. The maximum region of the throughput broadens more around the midpoint as the S-D SNR increases. The curves are symmetric around the midpoint.

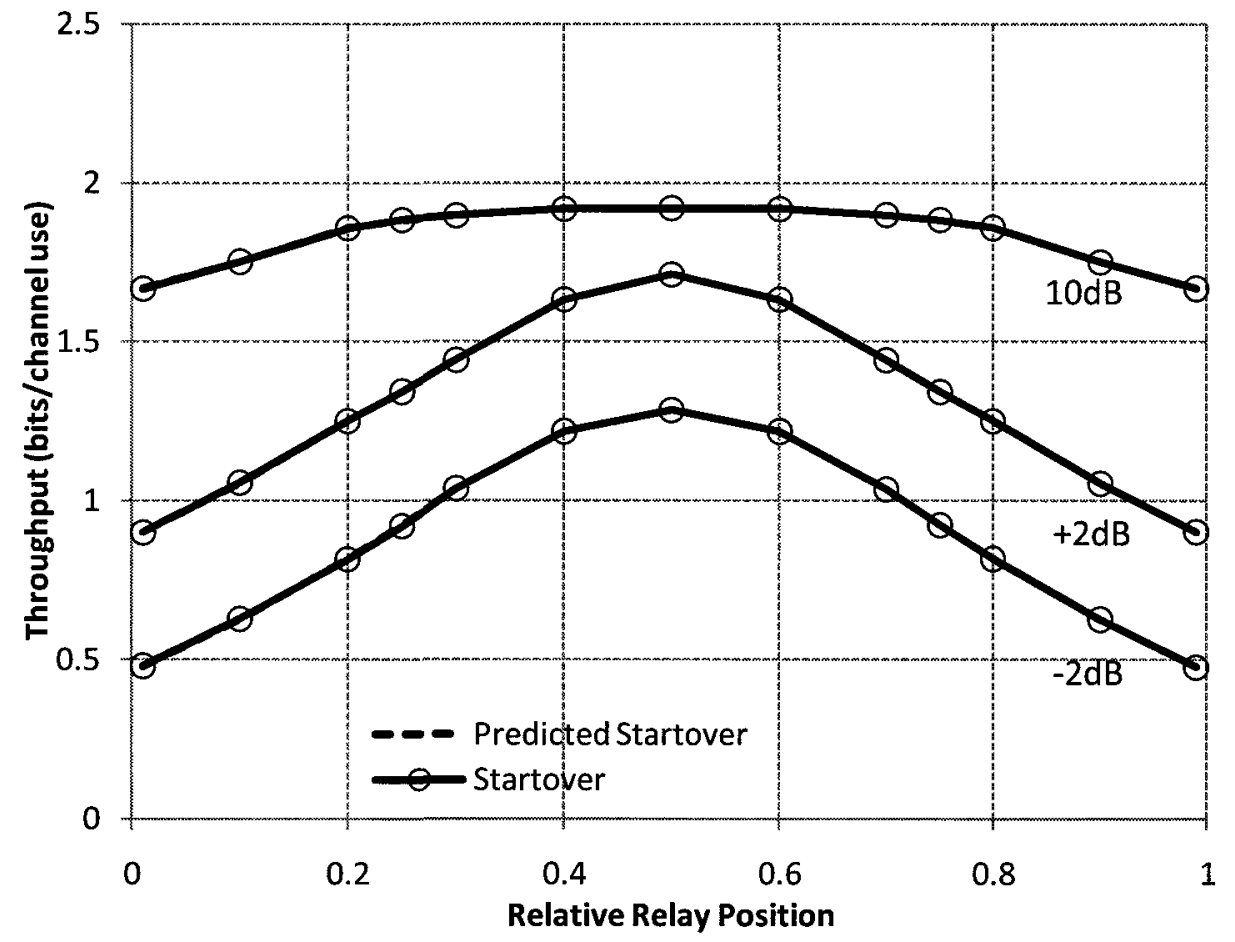

Figure 3.24: Theoretical throughput performance of startover relaying vs. actual throughput performance ( $K=384,16-Q A M)$. 


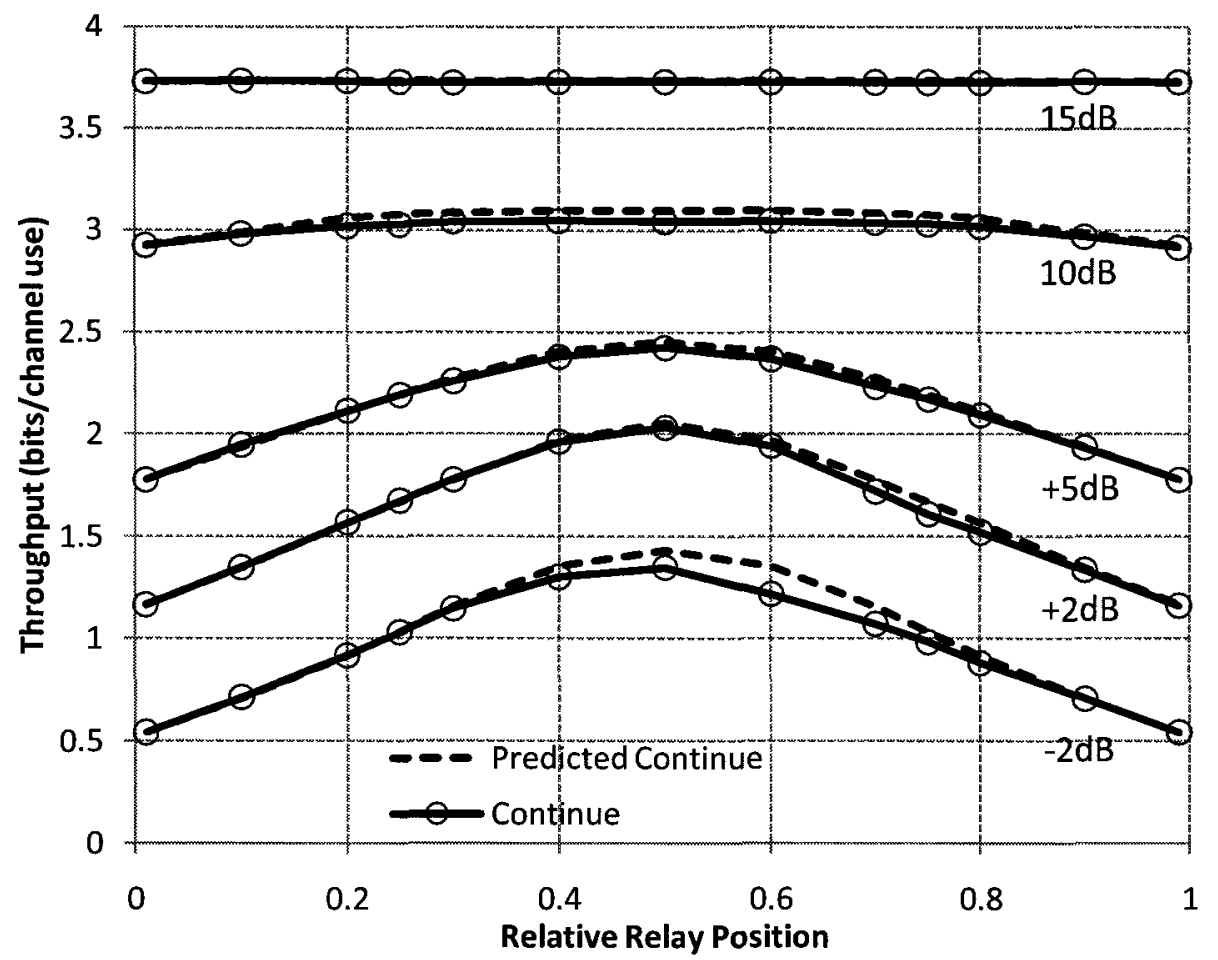

Figure 3.25: Theoretical throughput performance of continue relaying vs. actual throughput performance ( $K=384,16-Q A M)$.

Figure 3.25 compares the actual and the theoretical throughput performance of continue relaying. In general the two curves match very closely with slight errors, specifically, at the relative relay position 0.6 from the source for $\gamma_{S D}=-2 \mathrm{~dB}$. For 16 QAM, -2 dB is considered very low SNR. This error could be due to the code structure itself. In general, the two curves are considered matching, and the formula is considered applicable.

As the S-D SNR level increases, continue relaying throughput increases correspondingly until it saturates to the single-hop transmission. Still, continue relaying promises not to go below the single-hop throughput. It is similar to the BPSK results with a scale up factor. The throughput is almost symmetric around the midpoint. 


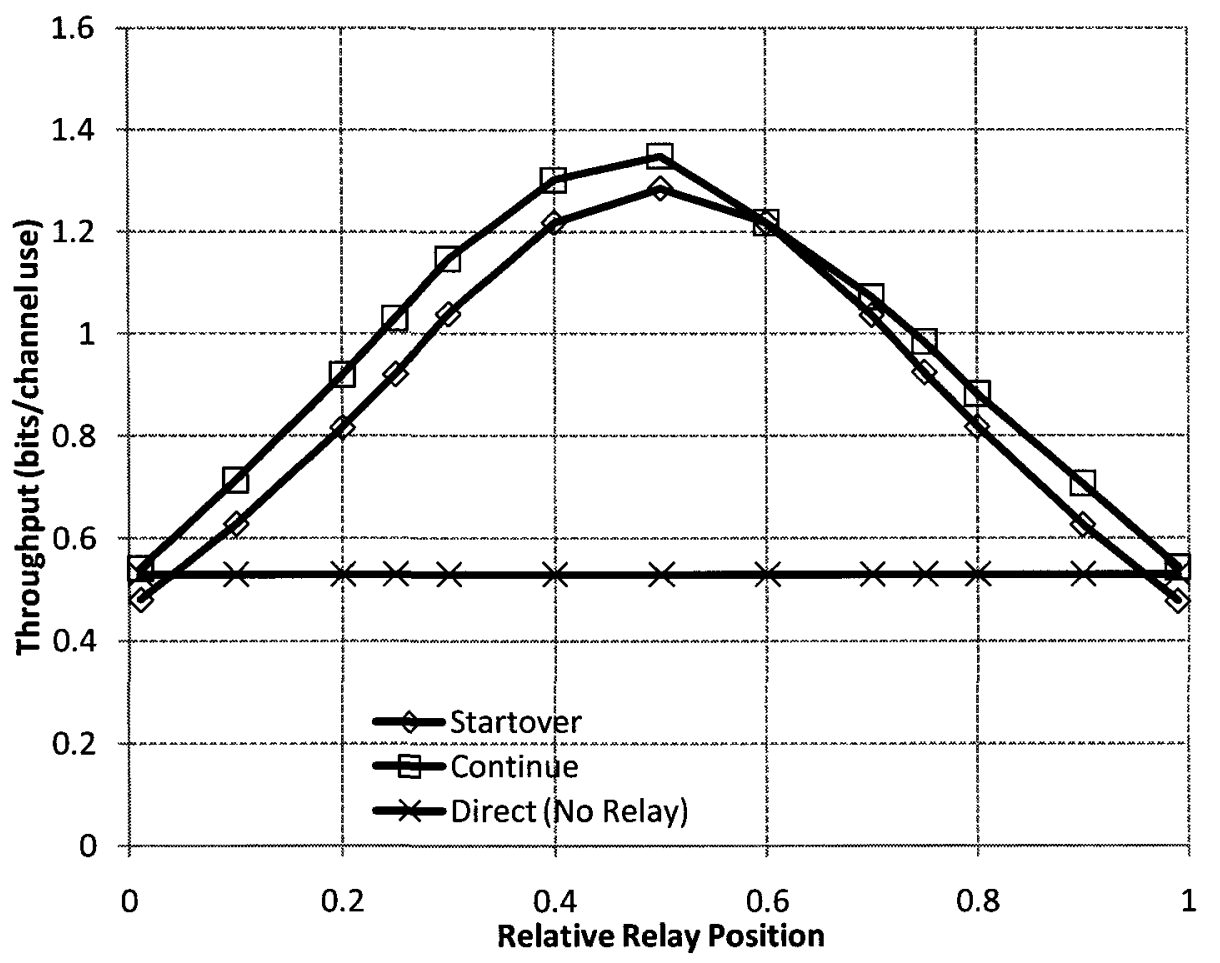

Figure 3.26: Comparison of linear relay performance: $K=384,16-Q A M, \gamma_{S D}=-2 d B$.

Figure 3.26 shows the two relaying modes throughput compared to the single-hop transmission throughput at a very low S-D SNR $(-2 \mathrm{~dB})$. The startover relaying forces its throughput to be below the single-hop when the relay is very close to the source or the destination. As explained earlier, it is due to the more code bits required to relay the codewords. The maximum throughput is very narrow and occurs at the midpoint. The startover relaying throughput is symmetric around the midpoint, and outperforms the single-hop throughput except when the relay is very close to the endpoints.

The continue relaying, as usual, surpasses both the startover relaying mode and the single-hop transmission. The difference between its throughput and the startover throughput is small since the S-D SNR is very low. The throughputs of the two relaying modes touch at the relative relay position 0.6 from the source. This creates a little asymmetry around the midpoint. This is due to the code structure. In general, one can 
assume it is still symmetric around the midpoint. Notice that the throughput never goes below the single-hop throughput.

The same comparison are shown in Figures 3.27 to 3.31 for higher S-D SNR levels. Both relaying throughput improves as the S-D SNR increases. The startover relaying throughput is always symmetric around the midpoint, while the continue relaying throughput is almost symmetric around the same point. The continue relaying always beat the startover relaying throughput.

As the S-D increases, the startover relaying throughput saturates to $1.92 \mathrm{bits} /$ channel use. However, the continue relaying maintains its throughput to be more than (at low SD SNRs) or equal to (at high S-D SNRs) the single-hop transmission throughput.

The throughput performances of the relaying modes behave similar to the BPSK cases. As so, it is evident that the relative performance of relaying using rateless coding does not depend on the modulation type. 


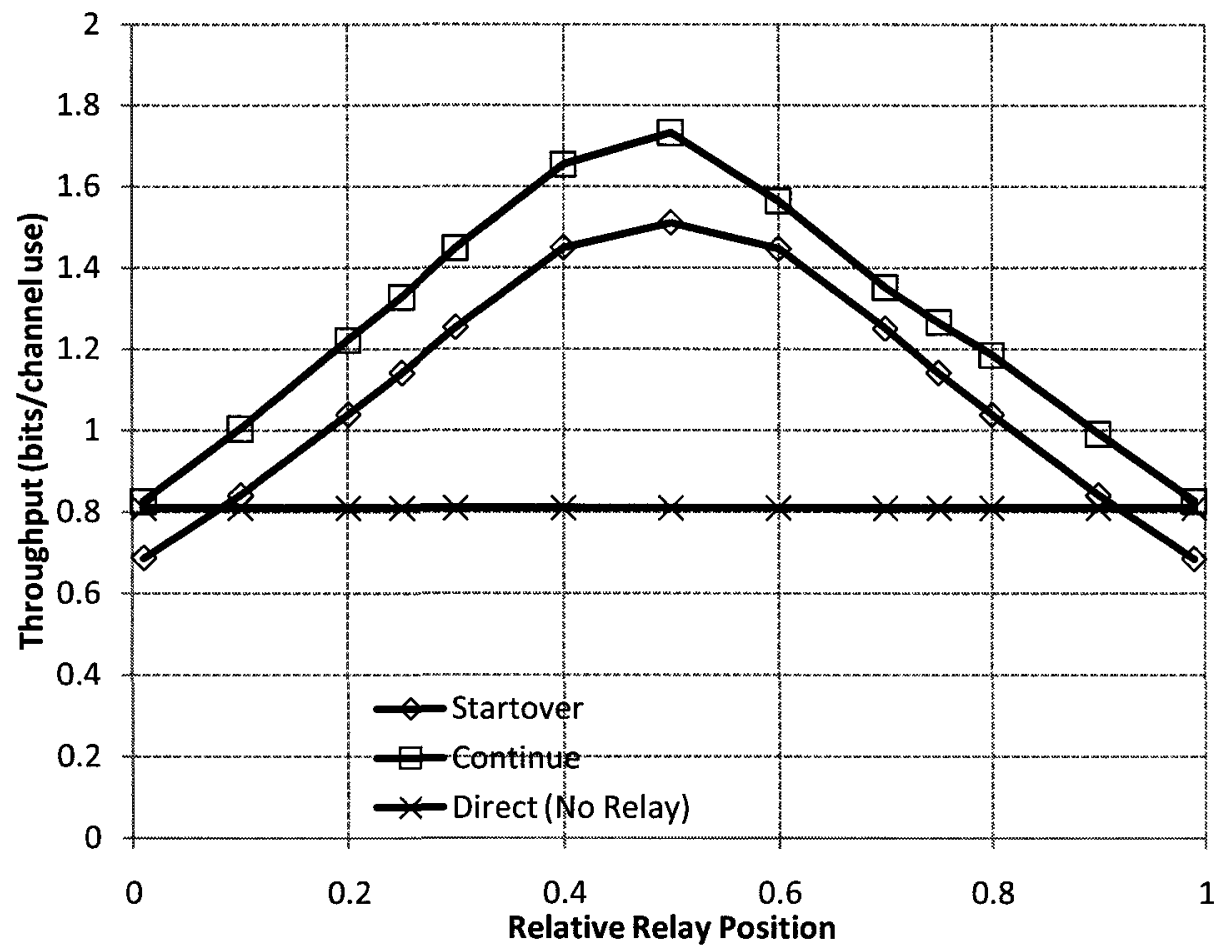

Figure 3.27: Comparison of linear relay performance: $K=384,16-\mathrm{QAM}, \gamma_{S D}=0 \mathrm{~dB}$.

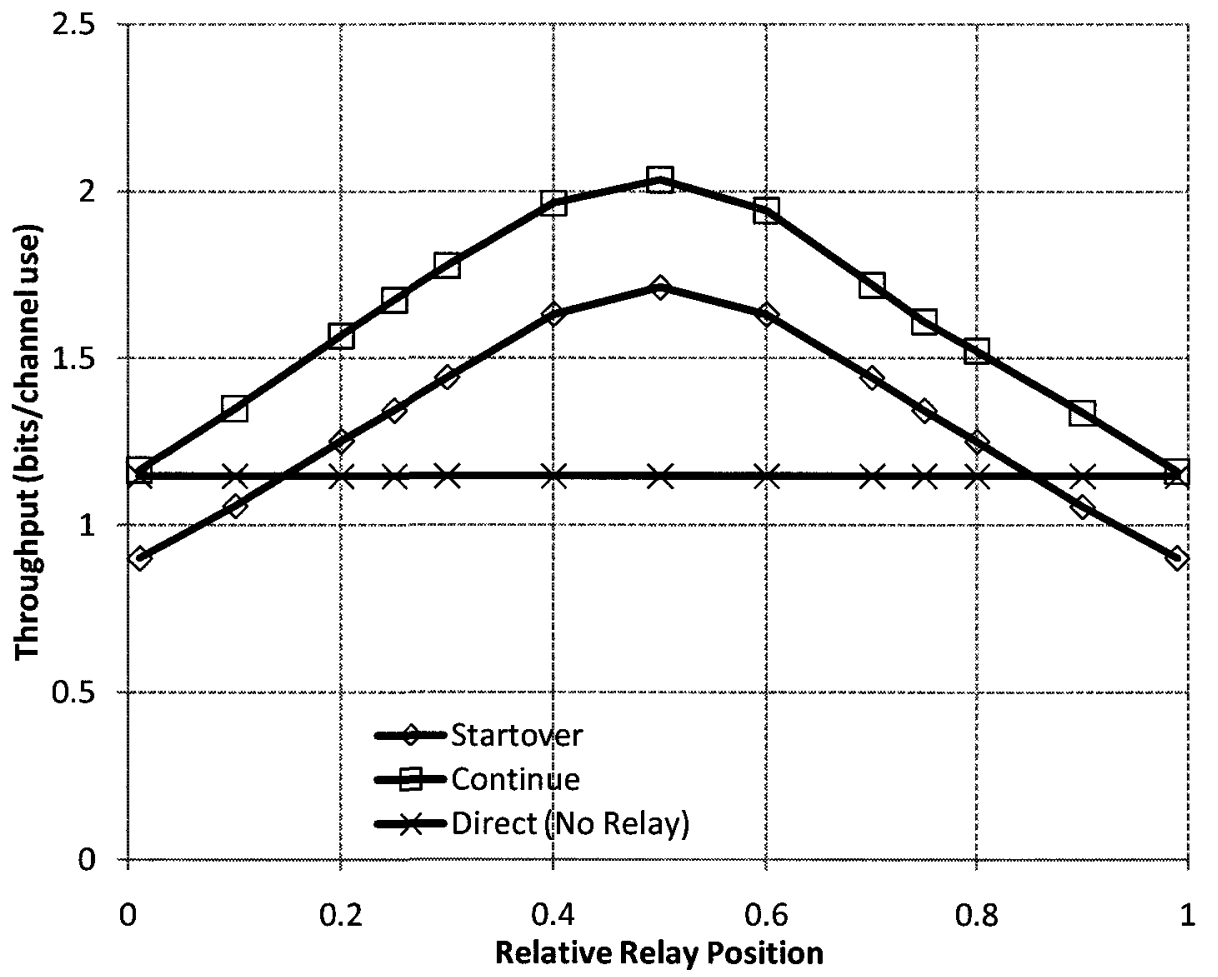

Figure 3.28: Comparison of linear relay performance: $K=384,16-\mathrm{QAM}, Y_{S D}=+2$ dB. 


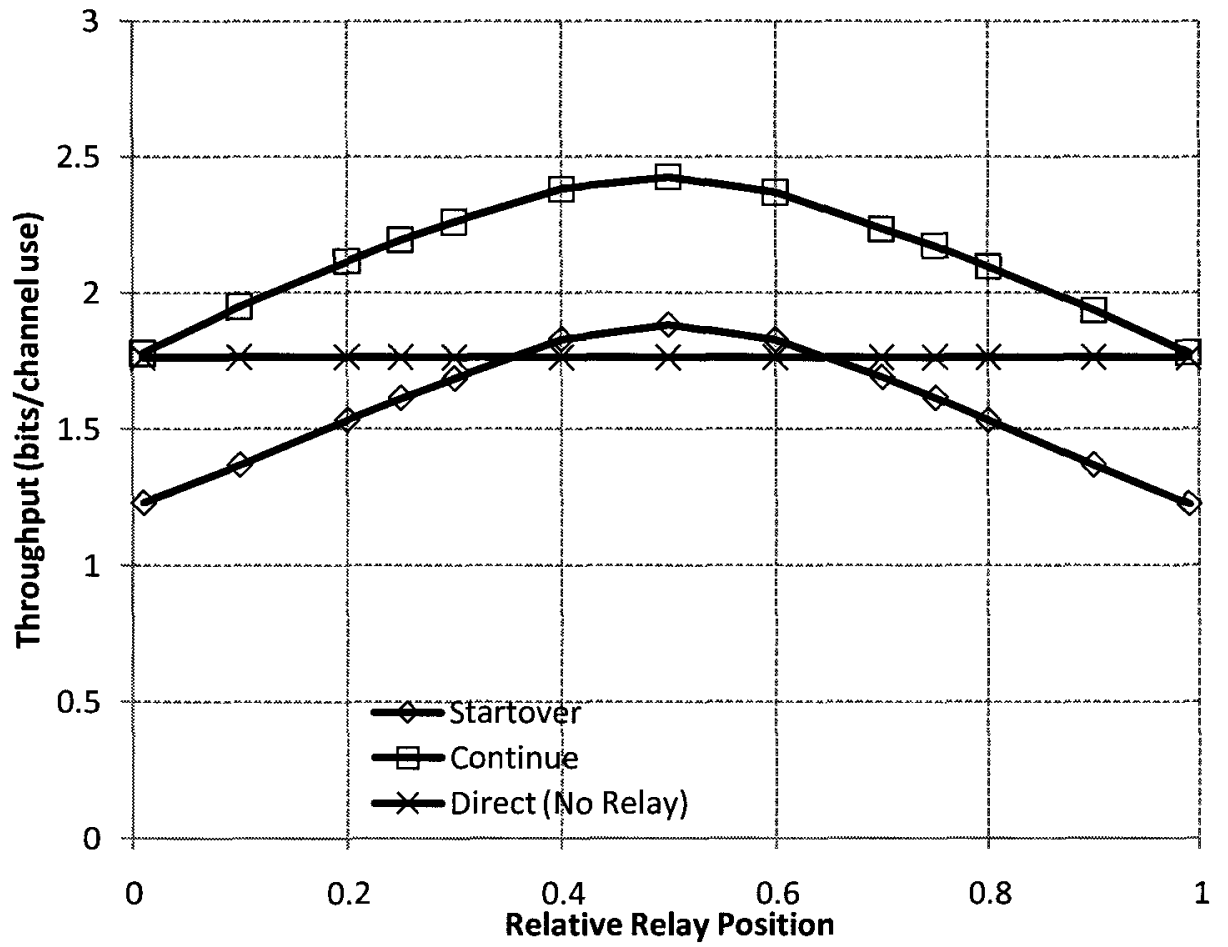

Figure 3.29: Comparison of linear relay performance: $K=384,16-\mathrm{QAM}, \gamma_{S D}=+5$ dB.

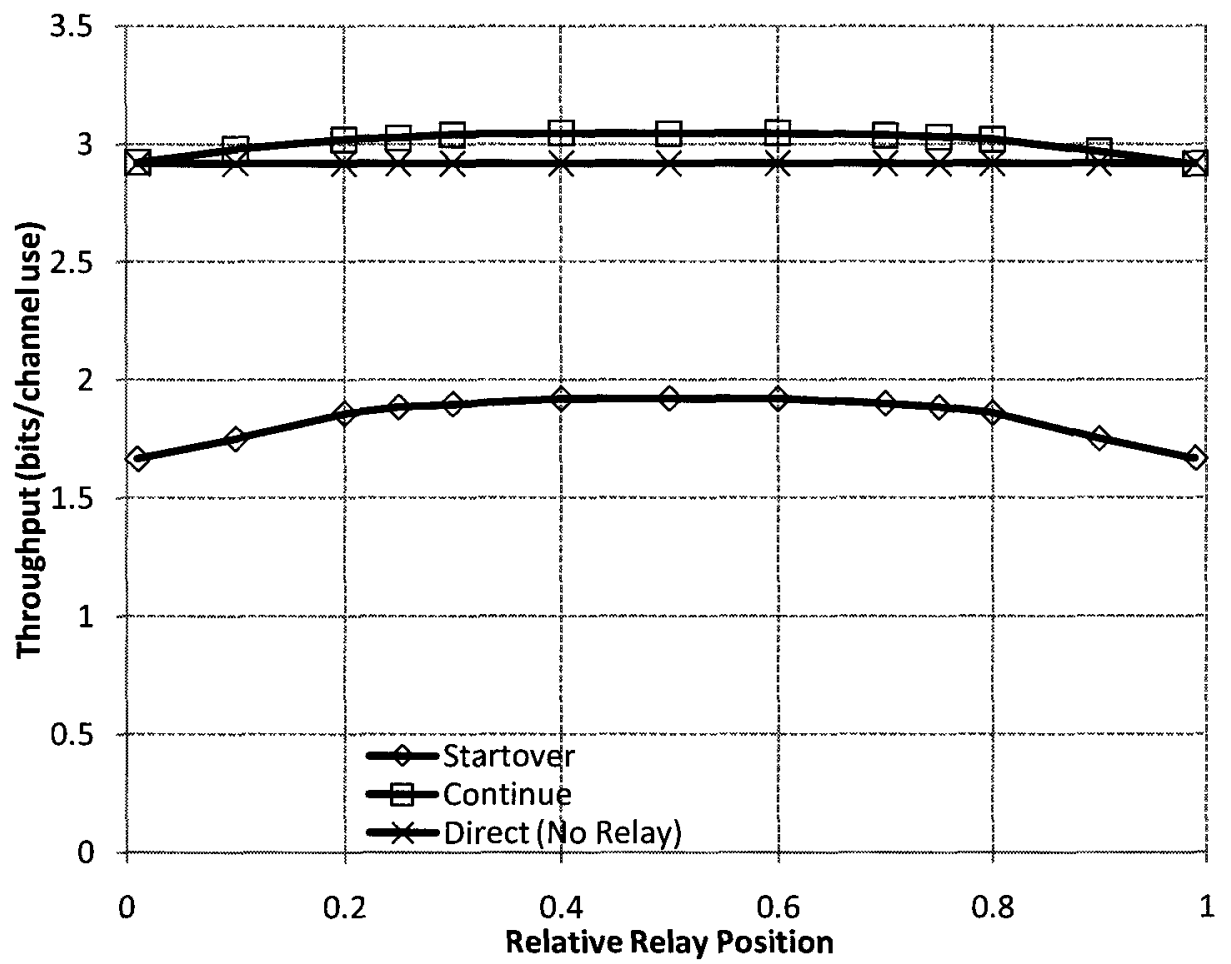

Figure 3.30: Comparison of linear relay performance: $K=384,16-\mathrm{QAM}, \gamma_{\mathrm{SD}}=+10$ dB. 


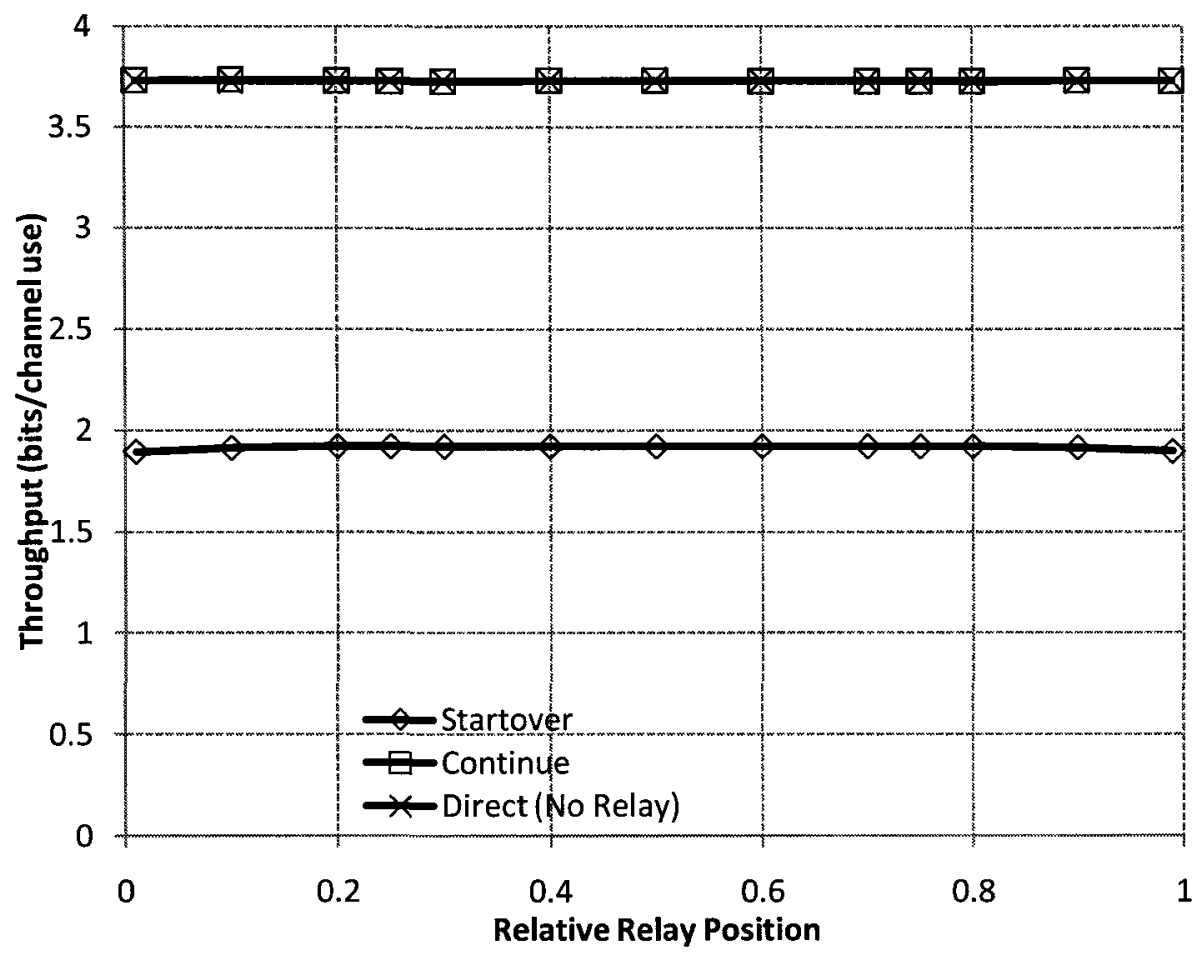

Figure 3.31: Comparison of linear relay performance: $K=384,16-\mathrm{QAM}, Y_{S D}=+15$ dB.

\subsubsection{Maximum Relaying Throughput of Startover and Continue}

To have an appreciation of the value gained when using relaying over a single-hop transmission, Figures 3.32 and 3.33 compare the maximum relaying throughputs with the single-hop throughput while assuming the relay is positioned in its best place, midways between the source and destination. It is evident that startover relaying performs better than single-hop at low SNRs only. The problem with startover relaying lies in the fact that even if the relay decodes faster than the destination, it is not necessarily true that the performance of relaying is better than single-hop transmission. The performance of the startover could be improved if the destination had full knowledge of the S-R CSI, so it could decide whether to relay or not. 
On the other hand, continue relaying performs better than the single-hop transmission (especially at low and mid transmitted powers) or at least the same (at high transmitted powers) as the single-hop. It is worth noting that the worse continue throughput performance (at low transmitted powers), is better than startover relaying. At extremely low transmitted power levels, continue relaying imitates the startover relaying. This is predicted by Equation (2.33), by letting $\eta_{S D}$ go to zero. The most attractive advantage of continue relaying over startover relaying is that continue relaying requires the least negotiation and it doesn't require the knowledge of the S-R CSI at destination to perform better.

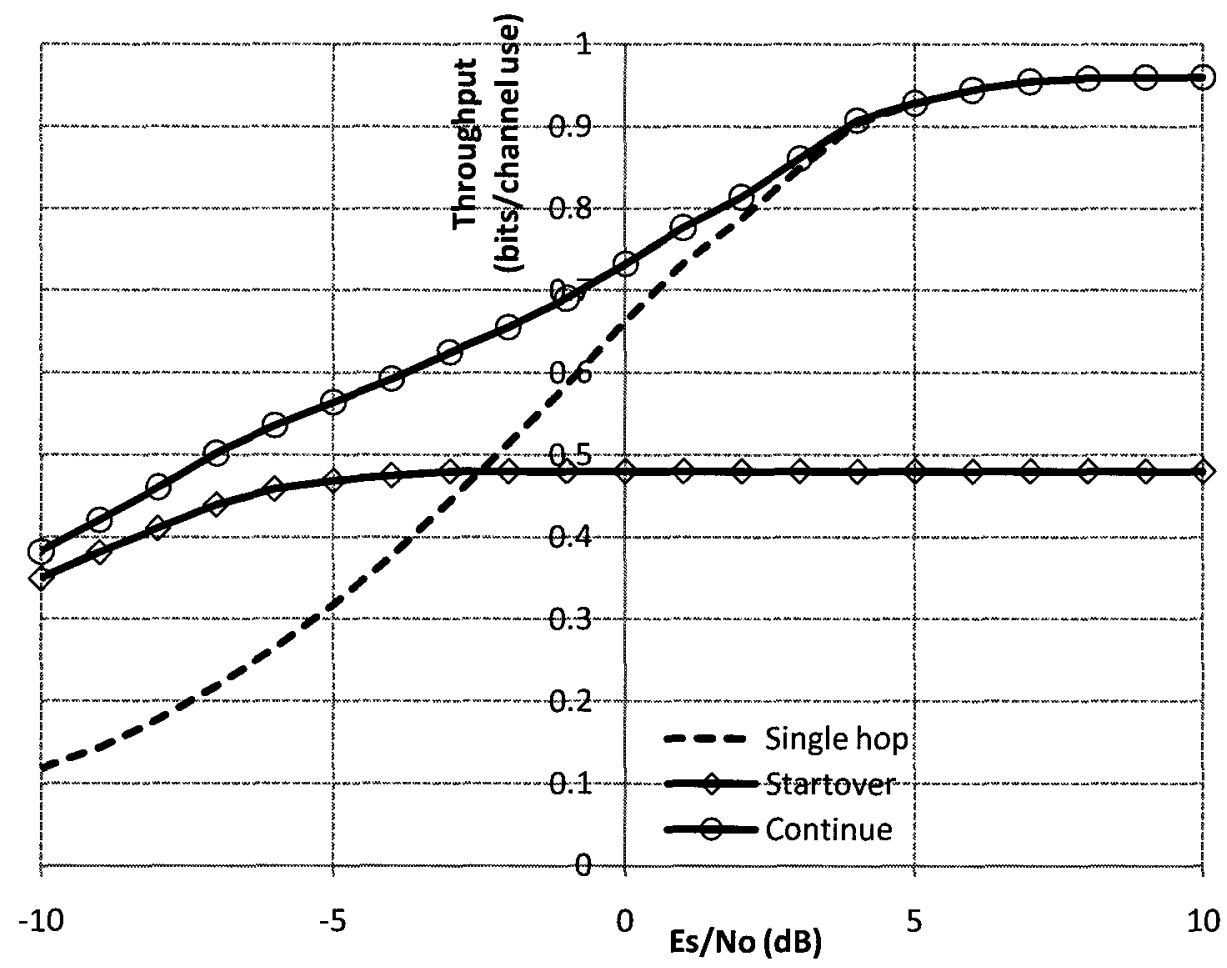

Figure 3.32: Optimum relaying vs. single-hop performance (BPSK). 


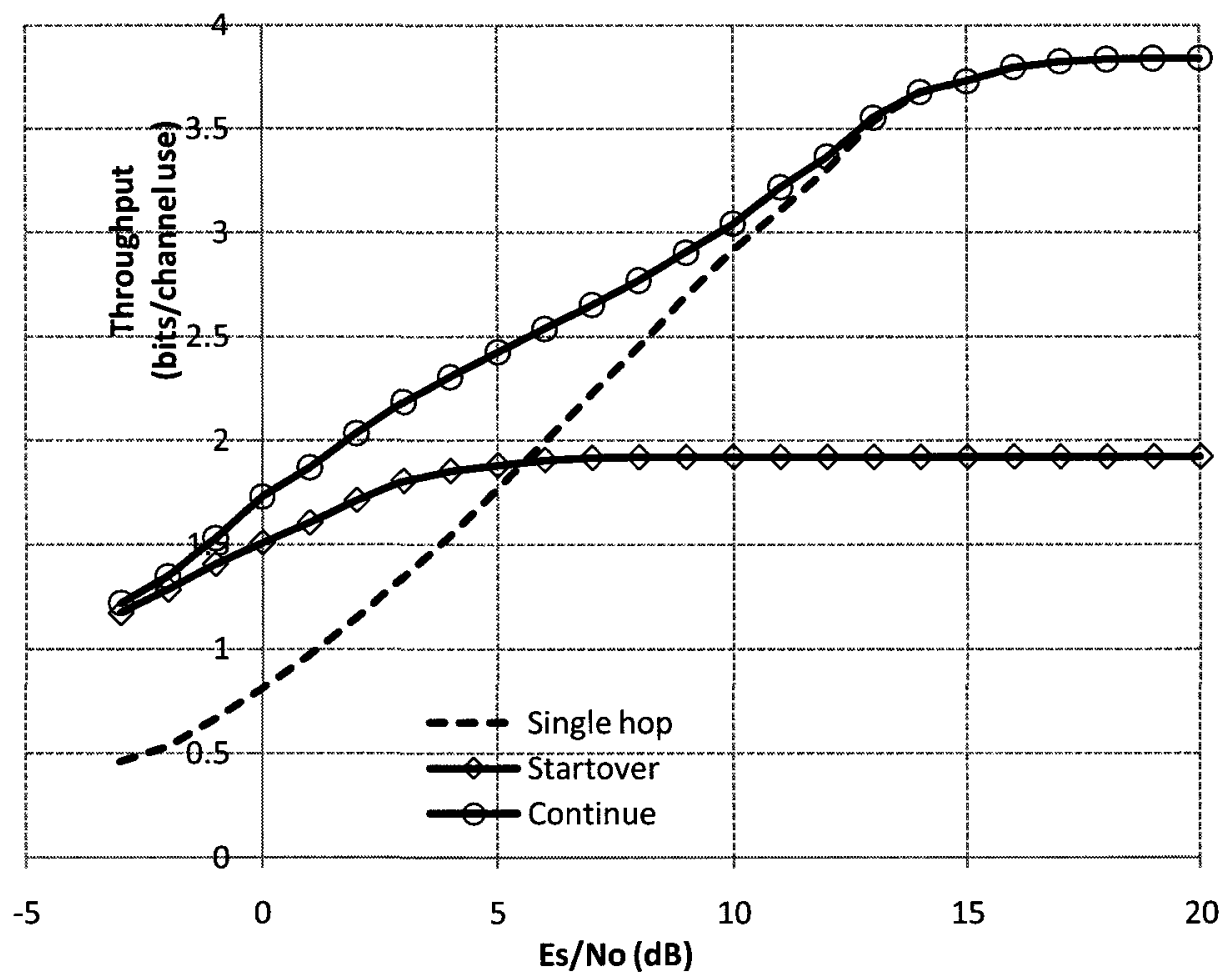

Figure 3.33: Optimum relaying vs. single-hop performance (16-QAM). 


\section{Application to Cellular Systems}

It is worthwhile to see the benefits of the proposed relaying scheme in a more realistic application. This chapter applies relaying to the popular wireless system: the cellular system. The system uses rateless coding, and non-simultaneous continue relaying. Two main goals of improving wireless systems are the enhancement of coverage and data rate. Although most of previous research finds these goals contradictory, in this chapter it will be demonstrated that coverage can be guaranteed without degrading the system average data rate. In fact, the data rate could be improved by using rateless-code continue relaying.

\subsection{Cellular System Model}

Cellular coverage is usually modeled using hexagonal shape cells with the base station in the middle. Each base station coverage area is called a cell. A technique that is widely used in cellular networks to increase the number of access channels is sectorization. Sectorization is basically splitting each cell into different sectors, where each sector operates at different carrier frequency to eliminate interference among adjacent cells while increasing the number of access channels. Figure 4.1 depicts the model of a hexagonal cell with three sectors. The solid lines represent the borders between sectors and the dashed lines represent the boresight of each sector antenna. 


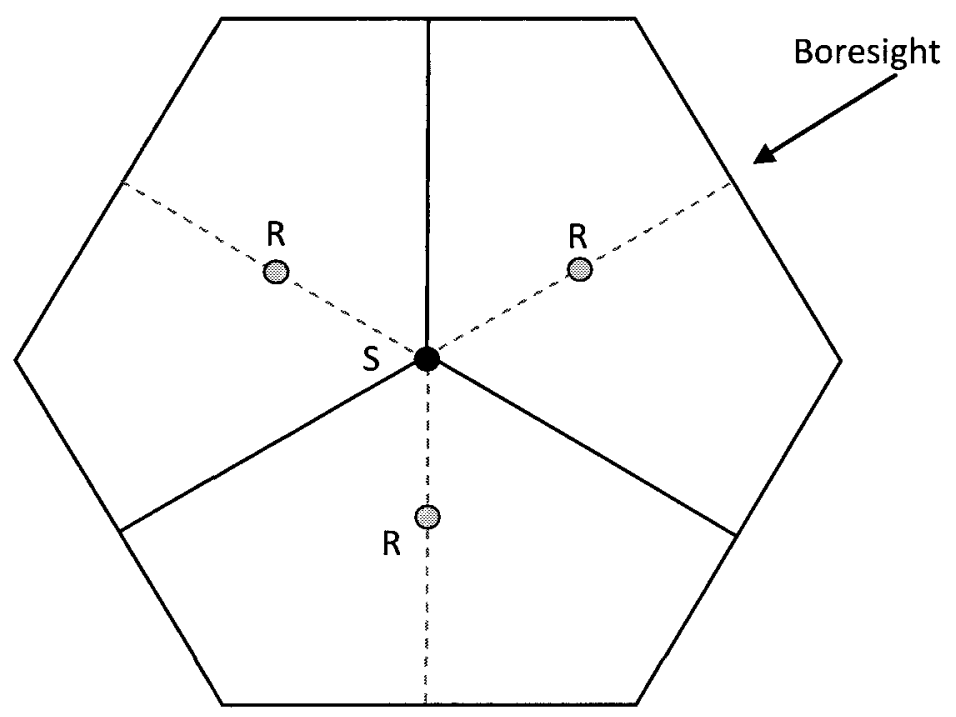

Figure 4.1: Cell sectors.

Each sector is illuminated by one directional antenna operating at a different carrier frequency from adjacent sectors. The directional antenna confines the bulk of its transmitted energy to the targeted sector. The boresight distance (shortest distance) from the base station to the border of the cell is $d_{\text {bore }}$.

There are three relays per cell used in this model; each relay is placed along the boresight line of each antenna. The distance between the base station to the relay is $d_{S R}$

Downlink and uplink channels operate in distinct frequency bands. The uplink channel is used here mainly to carry very brief messages like type II HARQ and relay association messages. The uplink channel is assumed reliable and available all the time, and doesn't suffer any impairment, as the research focuses on the downlink performance only. 
The downlink channel is modeled to take into account path losses, shadowing and AWGN noise. There are small scale impairments that involve multipath fading, and Doppler effects due to receiver/transmitter movement. The propagation model in this chapter ignores small scale impairments. Shadowing is taken into account because it is the main cause of coverage outages.

\subsubsection{Signal Power Model}

The power level of the received signal is modeled as

$$
P_{\text {rec }}=P_{\text {trans }}+G_{\text {trans }}(\theta)+G_{r e c}-L_{p}(d)-L_{s}
$$

where $P_{\text {rec }}$ is the received power $(\mathrm{dBm}), P_{\text {trans }}$ is the transmitted power $(\mathrm{dBm}), G_{\text {trans }}(\theta)$ is the transmitter antenna gain as a function of the angle relative to antenna boresight $(\mathrm{dB}), G_{r e c}$ is the receiver antenna gain (dB), $L_{p}(d)$ is the path loss as a function of distance separation between the transmitter and the receiver, $L_{s}$ is the shadowing loss power (dB), $d$ is the path distance (meter), and $\theta$ is the transmit angle relative to antenna boresight (degrees).

The path loss in $\mathrm{dB}$ is modeled as

$$
L_{p}(d)= \begin{cases}44.373 & ; \text { if } d<1 \text { meter } \\ 44.373+10 n_{p} \log (d) & ; \text { if } d \geq 1 \text { meter }\end{cases}
$$

where $n_{p}$ is the pathloss exponent.

The shadowing loss is a lognormally distributed random variable with zero mean and variance $\sigma_{S}^{2}$.

The base station antenna pattern is 


$$
G_{\operatorname{trans}(S)}(\theta)=G_{D A}-\min \left[12\left(\frac{\theta}{\theta_{3 d B}}\right)^{2}, A_{\max }\right]
$$

where $G_{D A}$ is the main lobe antenna gain (at $\theta=0$ ) in $\mathrm{dB}, \theta_{3 d B}$ is the $3 \mathrm{~dB}$ beam width, and $A_{\max }$ is the maximum attenuation (in $\mathrm{dB}$ ).

The transmitter antenna at the relay is assumed isotropic, with constant gain $G_{\text {trans }(R)}$. The receiver antenna at the relay and the destination are assumed isotropic too, with constant gains $G_{\operatorname{rec}(R)}$, and $G_{\operatorname{rec}(D)}$.

The SNR, $\gamma$, is calculated as the ratio of received power to the noise power

$$
\gamma=\frac{P_{r e c}(m W a t t)}{P_{N}(m W a t t)}=P_{r e c}(d B m)-P_{N}(d B m)
$$

where $P_{N}$ is the noise power $\left(P_{N}=N_{0} / 2\right)$. The noise power is simply the sum of the thermal noise $\left(N_{T}\right)$ power and the noise figure $\left(N_{F}\right)$

$$
P_{N}(d B m)=N_{T}+N_{F}
$$

\subsubsection{Simulation Algorithms}

At each simulation instance, three algorithms are run. The first algorithm is the "signal power evaluation" algorithm, which finds the received power level at both the relay and the destination. The second algorithm is the "relay association" algorithm, which performs the relay association between the destination and the sector relay. The third algorithm is the "codeword transmission" algorithm, which runs the transmission and decoding processes to find the total number of bits required to convey the message to the destination.

The signal power evaluation algorithm is summarized as follows: 
1. The three relays are placed on the boresight lines of the three sector antennas, at a distance $d_{S R}$.

2. The end terminal (D) is placed randomly in the cell.

3. The distances $d_{S D}$, between the base station (S) and the destination (D), and $d_{R D}$, between the destination (D) and the sector relay (R), are calculated.

4. The transmitted powers at the base station $\left(P_{\operatorname{trans}(S)}\right)$ and the relay $\left(P_{\operatorname{trans}(R)}\right)$ are set to their desired values.

5. Three samples are generated corresponding to shadowing power losses for the three channels S-D, S-R, and R-D. Each sample is lognormally distributed with mean zero and standard deviation $\sigma_{S}$.

6. Path losses are computed for the three channels, using (4.1).

7. The SNR ratios are calculated for the three channels, using (4.4).

Once the three channel SNRs are known, the next step is the relay association. The relay association only occurs if the SNR of the R-D channel is higher than the SNR of the S-D channel. Practically, SNRs can be easily found at the destination receiver by sending frequent training messages between the three devices. As so, the relay association is conducted as follows:

1. If $\gamma_{S D}<\gamma_{R D}$, then the destination sends the RelayAssoc message and both the base station and the relay receive it through the uplink channel.

2. If $\gamma_{S D} \geq \gamma_{R D}$, then no association message is sent and so no relaying will occur.

Now the codeword transmission takes place. The codeword transmission algorithm is run as follows: 
1. The information messages are generated randomly with each bit equally likely to be zero or one. The message sizes are of length $K$.

2. Messages are passed to the RC/QC-LDPC encoder, generating codewords of length $N_{\max }$.

3. The codewords are split into packets. The first packet of each codeword is of length $K$, and the consequent packets are of step size $L_{\text {inc }}$.

4. The bits of the codeword packets are mapped into BPSK signals having an averageenergy-per-bit equal to $E_{s}$.

5. The attenuation factors are calculated based on the channel SNRs found earlier, using Equation

$$
\alpha^{2}=\frac{2 P_{N}}{E_{S}} 10^{\left(\frac{\gamma}{10}\right)} \text {. }
$$

6. The received signals are computed by multiplying the transmitted signal by the attenuation factors, and adding them to the AWGN samples using Equations (2.13), (2.14), and (2.15).

7. The receivers at the relay and the destination start decoding after receiving the first packet of size $K$.

8. The decoders generate bit LRs of the received signals and start decoding. The LRs are computed using Equation (2.6).

9. If decoding is not successful at either receiver, more packets are transmitted with a step size of $L_{\text {inc }}$.

10. Every newly received packet is appended to the previously received packets in the decoder and decoded again. 
11. If the destination decodes first, then the transmission is announced successful and the destination sends back ACK message that is successfully received by the base station and the relay which ends the transmission session.

12. If the relay decodes faster than the destination, an ACK message is sent to the base station. If a relay association has already been established between the relay and the destination, the base station will stop transmitting and the relay will take over. Otherwise, the base station would continue transmitting.

13. If relaying is taking place, the relay would continue from where the base station stopped, until the destination decodes successfully or the maximum number of code bits are reached $\left(N_{\max }\right)$.

14. If the destination decodes successfully, it will send ACK message to end the transmission session.

15. In either case (relaying or not relaying), if the total number of transmitted bits reaches $N_{\max }$ then the transmission is considered failed and the destination is experiencing an outage.

The total number of transmission sessions is $T$. The performance of the system is measured by calculating the throughput and the number of outages. The outage probability is found be adding all unsuccessful transmissions, and divide them by the total number of transmission sessions

$$
\text { Outage Probability }=\frac{\sum \text { Outages }}{T}
$$

As for the throughput, it is found as 


$$
\eta_{\text {cell }}=\frac{T K}{\sum_{i=1}^{T} N(i)} .
$$

\subsection{Simulation Results}

This section presents the simulation results for the one-cell relay system, using continue relaying and RC/QC-LDPC coding. The system simulation parameter values are summarized in table 4.1 .

\begin{tabular}{|l|l|}
\hline Parameter & Value \\
\hline Modulation & BPSK \\
\hline$K$ & 384 bits \\
\hline$Z_{B}$ & 192 bits \\
\hline$N_{\max }$ & 3840 bits \\
\hline$n_{p}$ & 3.5 \\
\hline$E_{S}$ & 1 Joule \\
\hline$L_{\text {inc }}$ & 16 bits \\
\hline$d_{\text {bore }}$ & 750 meters \\
\hline$d_{S R}$ & $250 \mathrm{~m}, 300 \mathrm{~m}, 375 \mathrm{~m}, 450 \mathrm{~m}, 500 \mathrm{~m}, 535 \mathrm{~m}$ \\
\hline$P_{\text {trans }(S)}$ & $P_{S}$ \\
\hline$P_{\text {trans }(R)}$ & $P_{S}$ \\
\hline$P_{S}$ & $20 \mathrm{dBm}, 25 \mathrm{dBm}, 30 \mathrm{dBm}, 35 \mathrm{dBm}, 40 \mathrm{dBm}$ \\
\hline$\sigma_{S}$ & $8 \mathrm{~dB}$ \\
\hline$G_{D A}$ & $14 \mathrm{~dB}$ \\
\hline$\theta_{3 d B}$ & $70 \mathrm{degrees}$ \\
\hline$A_{\max }$ & $20 \mathrm{~dB}$ \\
\hline$G_{\text {trans }(R)}$ & $9 \mathrm{~dB}$ \\
\hline$G_{\text {rec }(R)}$ & $9 \mathrm{~dB}$ \\
\hline$G_{\text {rec }(D)}$ & $9 \mathrm{~dB}$ \\
\hline$N_{T}$ & $-101.8403 \mathrm{dBm}$ \\
\hline$N_{F}$ & $+5 \mathrm{~dB}$ \\
\hline
\end{tabular}

Table 4.1: Simulation parameters for the one cell relay model.

The transmitted power at the base station and the relay are set equal. The simulation was run for 20,000 times at different power levels, and different relay positions.

There are different factors that control the optimum relay position. The relay position has to be close enough to the base station to be able to receive strong signals 
and decode faster. It also needs to be close enough to most end users so it can convey strong signals to them. If the end users are uniformly distributed over the cell, then the best relay positions would be closer to the cell borders. As the shadowing and the path losses are introduced, the farther users from the base station would suffer more from channel impairments. This dictates that the relay should be placed closer to the border of the cell. Although relay position optimization is not the research objective, the simulation surveys a few positions that likely to be good and compares the results.

Starting with the results of $P_{S}=20 \mathrm{dBm}$, Figure 4.2 shows the throughput performance of the cell-relay model. The relay position is changed from 250 meters to 525 meters in gradual steps. The system throughput is enhanced by around $50 \%$ by using the relays. The relaying throughput slowly changes with the relay position, with an average throughput of about $0.41 \mathrm{bits} / \mathrm{channel}$ use. The optimum relay position is about in the middle distance from the cell border to the base station ( 375 meters). Actually at this low power level, the optimum relay position is expected to be closer to the base station. Yet, when the relay is close to the base station it wastes more transmissions because of the high number of outages. Hence, the optimum relay position shifts to the middle. Notice that the single-hop transmission throughput is around $0.26 \mathrm{bits} / \mathrm{channel}$ use. 


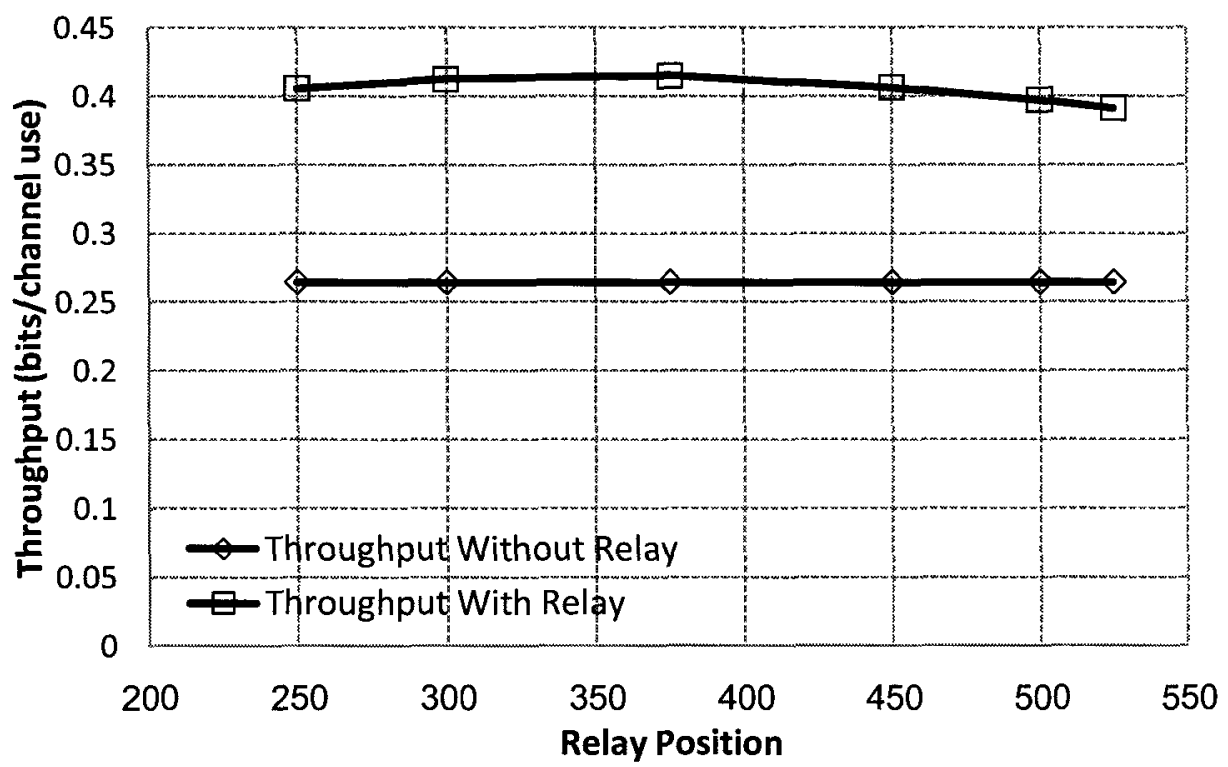

Figure 4.2: Cell throughput performance $\left(P_{s}=20 \mathrm{dBm}\right)$.

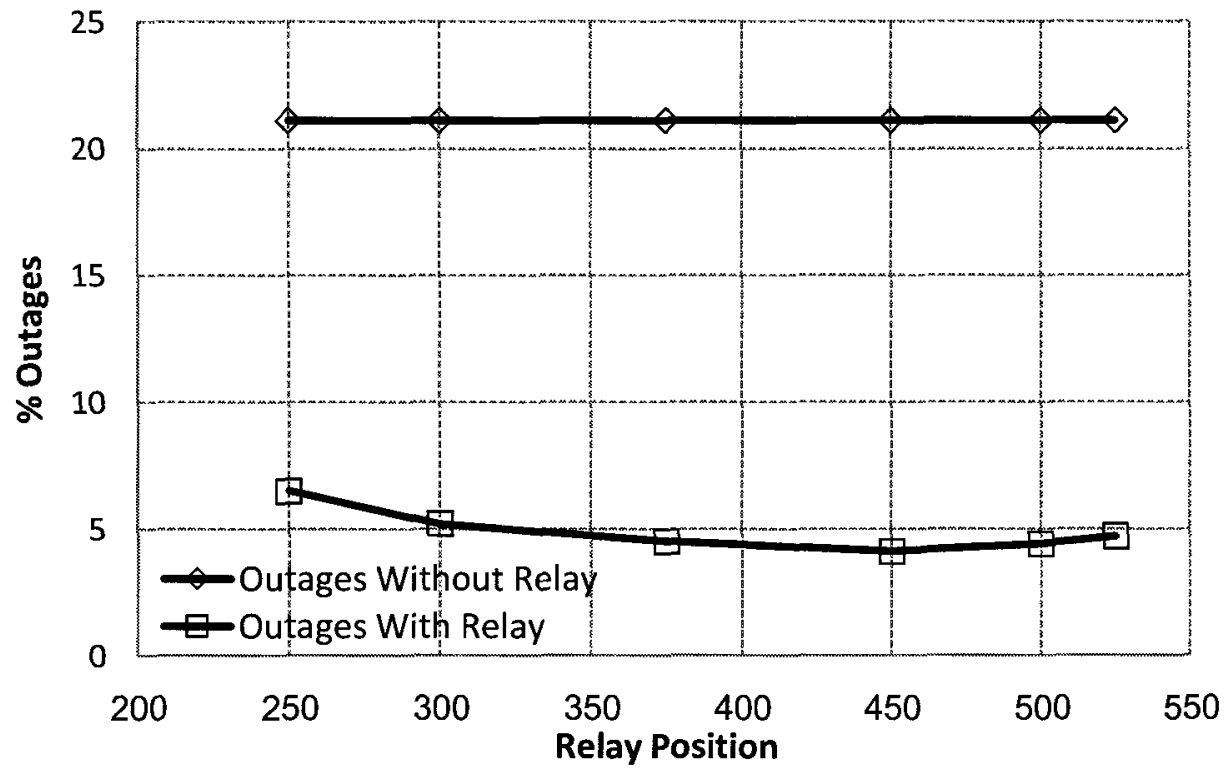

Figure 4.3: Outage probabilities $\left(P_{s}=20 \mathrm{dBm}\right)$.

The outage probability is shown in Figure 4.3. The minimum outage probability occurs at the distance of 450 meters from the base station. The optimum relay position decreases the outage probability by about $80 \%$. The average outage probability is $21 \%$ for the single-hop transmission, and $5 \%$ for the relayed system. 
As the transmitted power increases, the overall throughput and outage probability improve. Figure 4.4 shows the throughput performance of both single-hop and relayed system at transmitted power level $P_{S}=25 \mathrm{dBm}$. The average throughput is 0.39 bits/channel use for the single-hop, and 0.62 bits/channel use for the relayed system. Relaying enhances the transmission throughput by about $58 \%$ over single-hop transmission. The maximum relaying throughput is about 0.63 bits/channel use at 375 meters from the base station. The maximum is close to the average throughput of the relayed system.

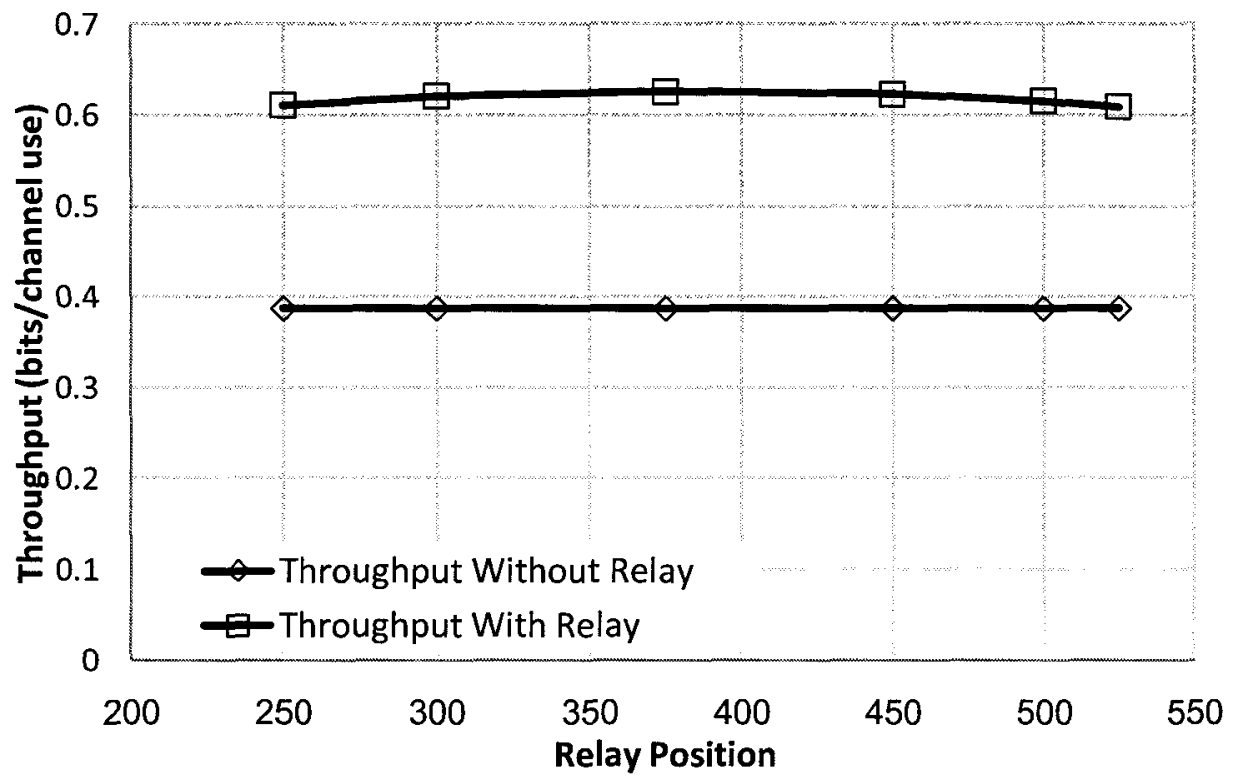

Figure 4.4: Cell throughput performance $\left(P_{s}=25 \mathrm{dBm}\right)$.

The outage probability for $P_{S}=25 \mathrm{dBm}$ is shown in Figure 4.5. The optimum relay position is right at $\mathbf{3 7 5}$ meters from the base station. The average outage probability for the relayed system is about $4 \%$, while it is $9.7 \%$ for single-hop transmissions. The outage enhancement of using relays is about $50 \%$. 
Further simulations are done at higher transmitted power levels: $30 \mathrm{dBm}$, and 35 $\mathrm{dBm}$. The results are depicted in Figures 4.6 to 4.9. The advantage of using relays decreases as the transmitted power increases.

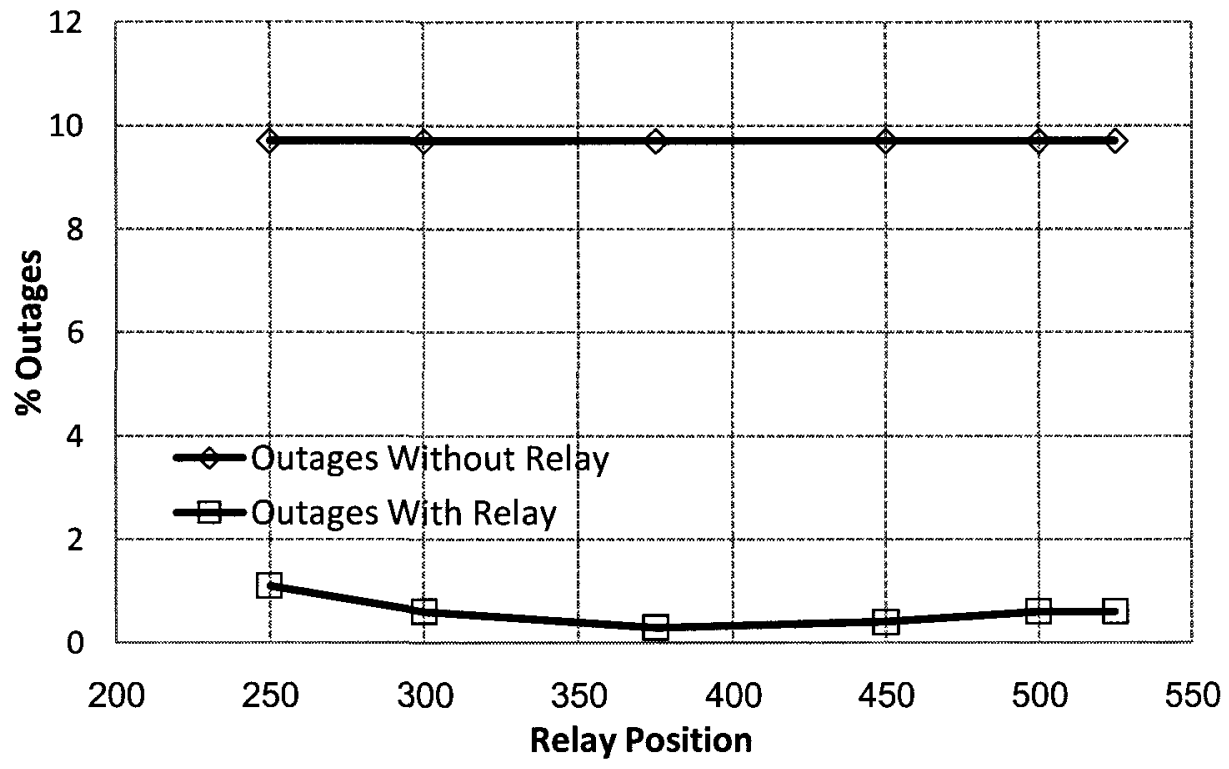

Figure 4.5: Outage probabilities $\left(P_{s}=25 \mathrm{dBm}\right)$.

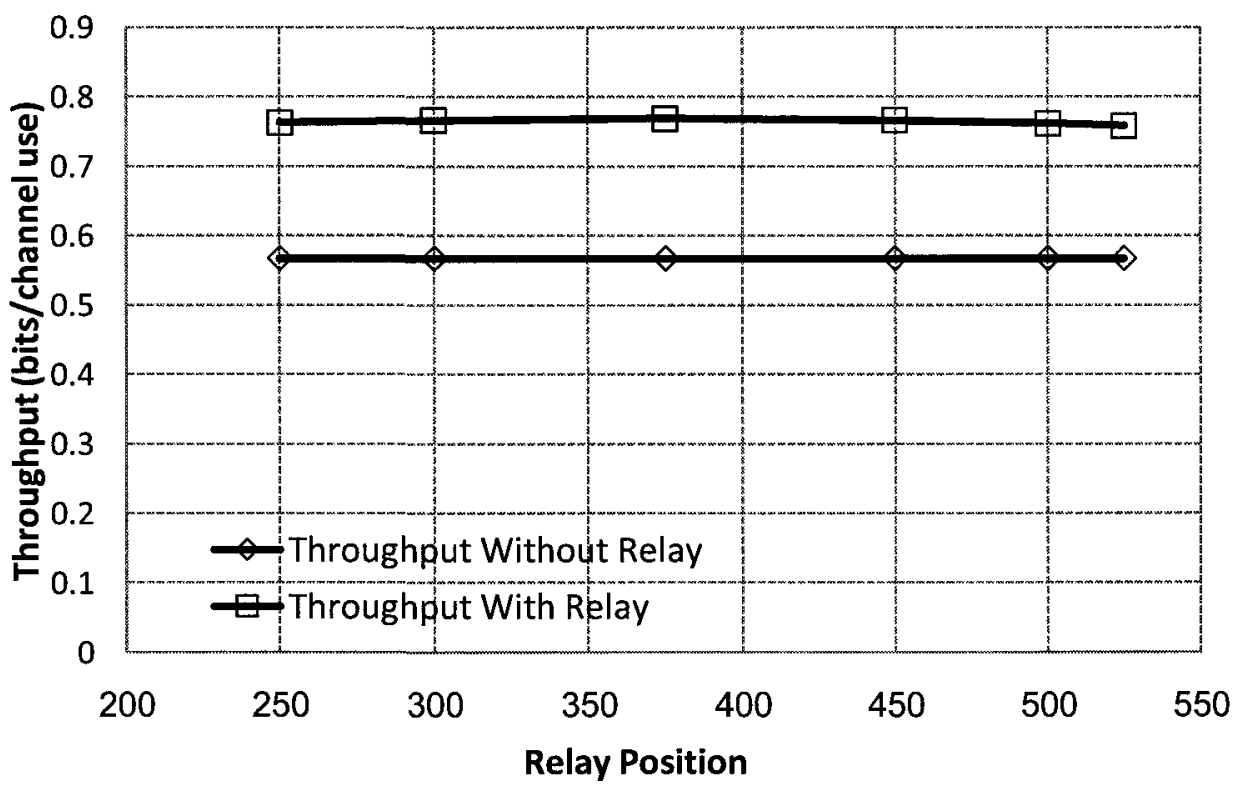

Figure 4.6: Cell throughput performance $\left(P_{s}=30 \mathrm{dBm}\right)$. 


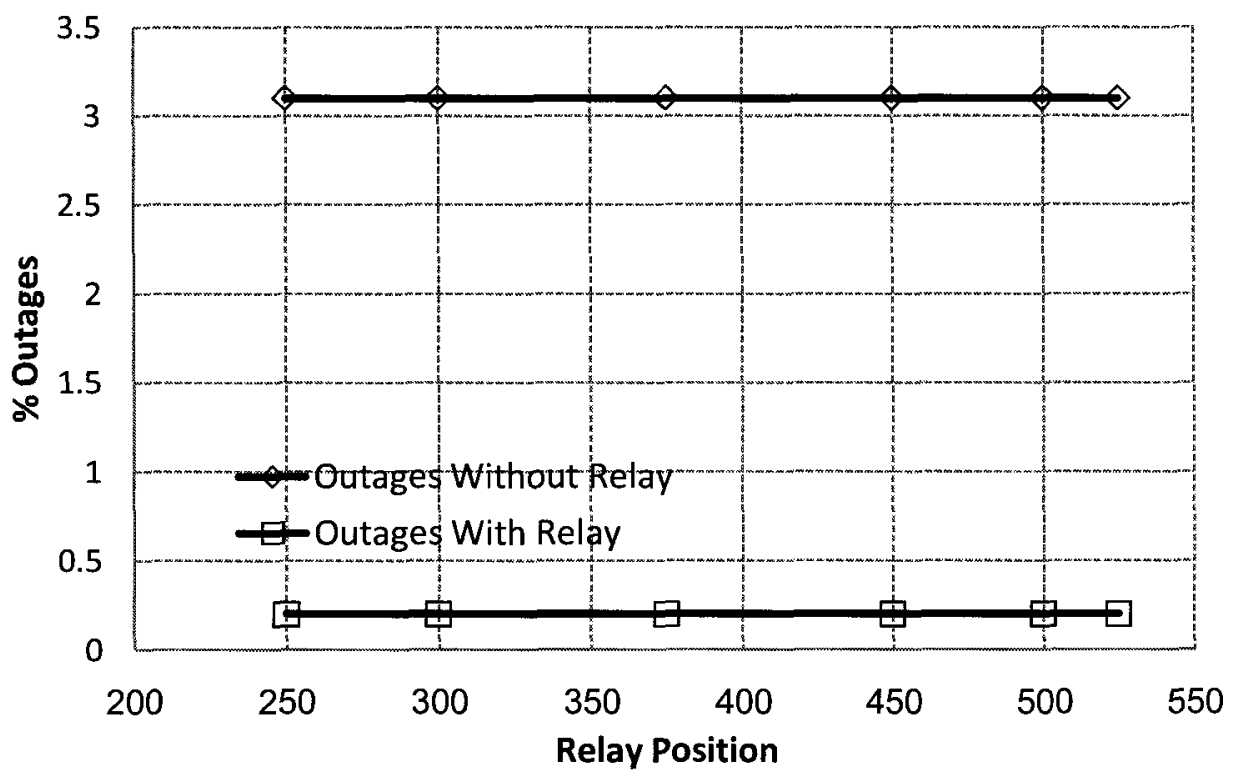

Figure 4.7: Outage probabilities $\left(P_{s}=30 \mathrm{dBm}\right)$.

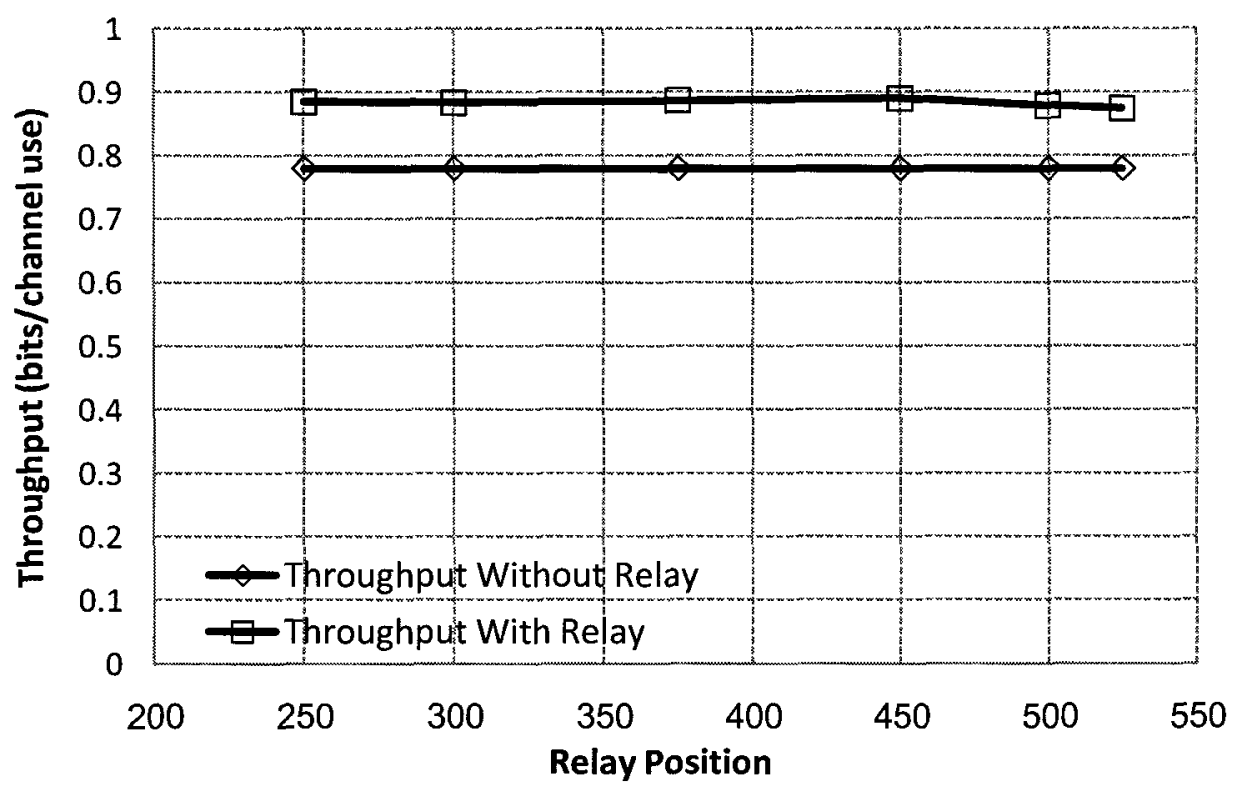

Figure 4.8: Cell throughput performance $\left(P_{s}=35 \mathrm{dBm}\right)$. 


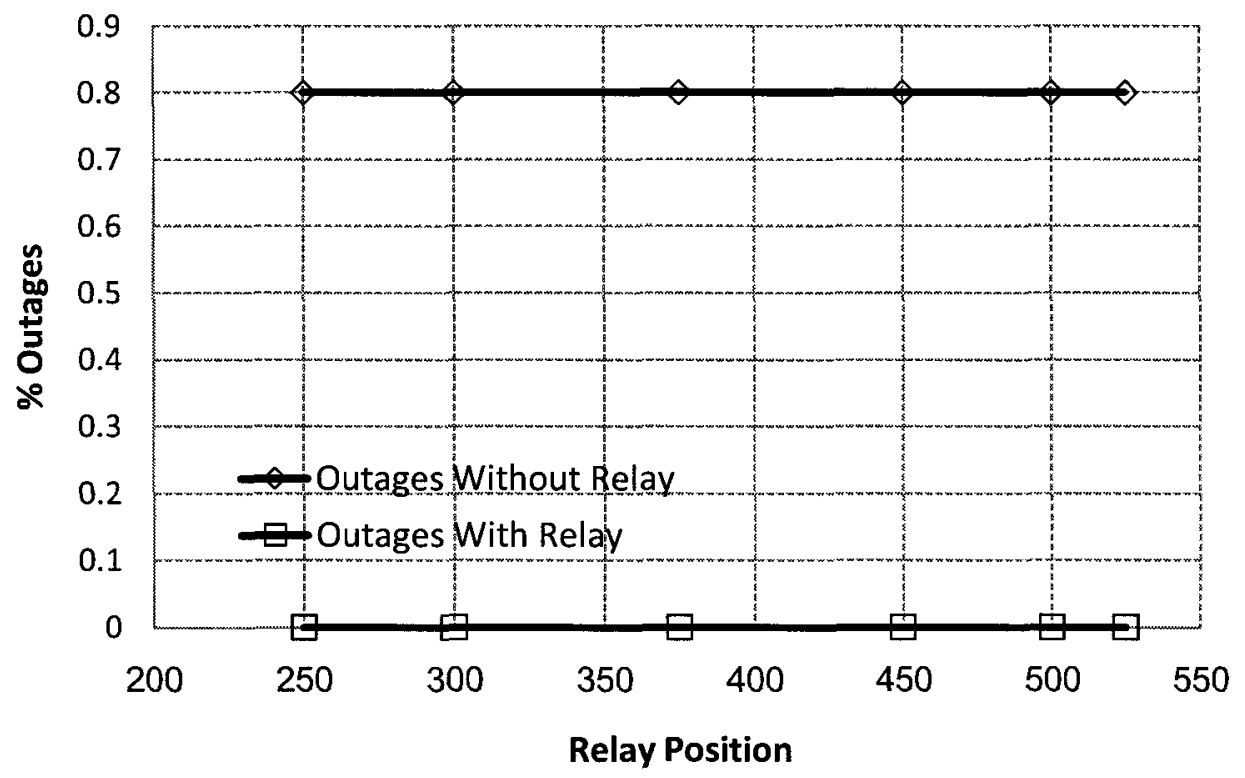

Figure 4.9: Outage probabilities $\left(P_{s}=35 \mathrm{dBm}\right)$.

Figures 4.10 compares the maximum average data rate delivered versus the transmitted power. Figure 4.11 shows the comparison of the minimum outage probability versus the transmitted power. The outage probability decreases sharply as power increases, for both direct single-hop transmissions and relaying. The relays can improve outage probability better than increasing the transmitted power. It is apparent that for low powered wireless cellular systems, and by having three relays in a cell is about equivalent to increasing the power by $10 \mathrm{~dB}$. Continue relaying proves to be an efficient way to reduce outages and achieve higher throughput. 


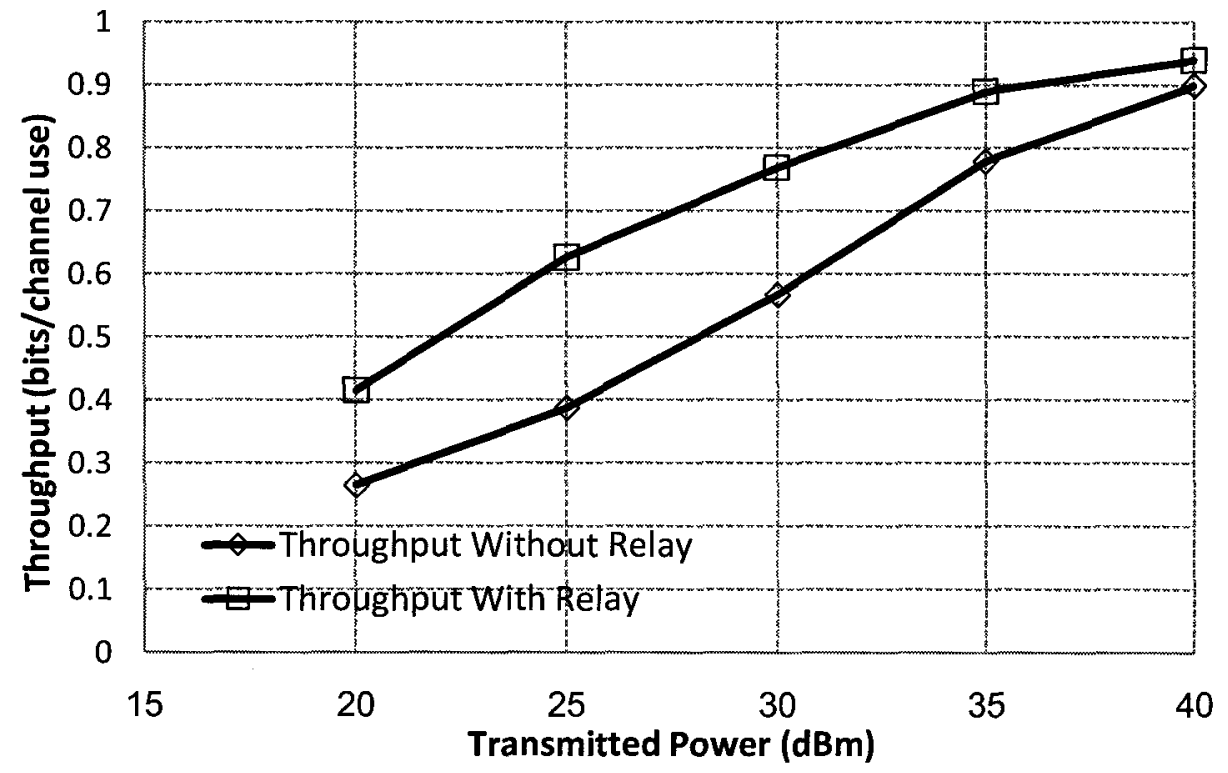

Figure 4.10: Maximum cell throughput profile

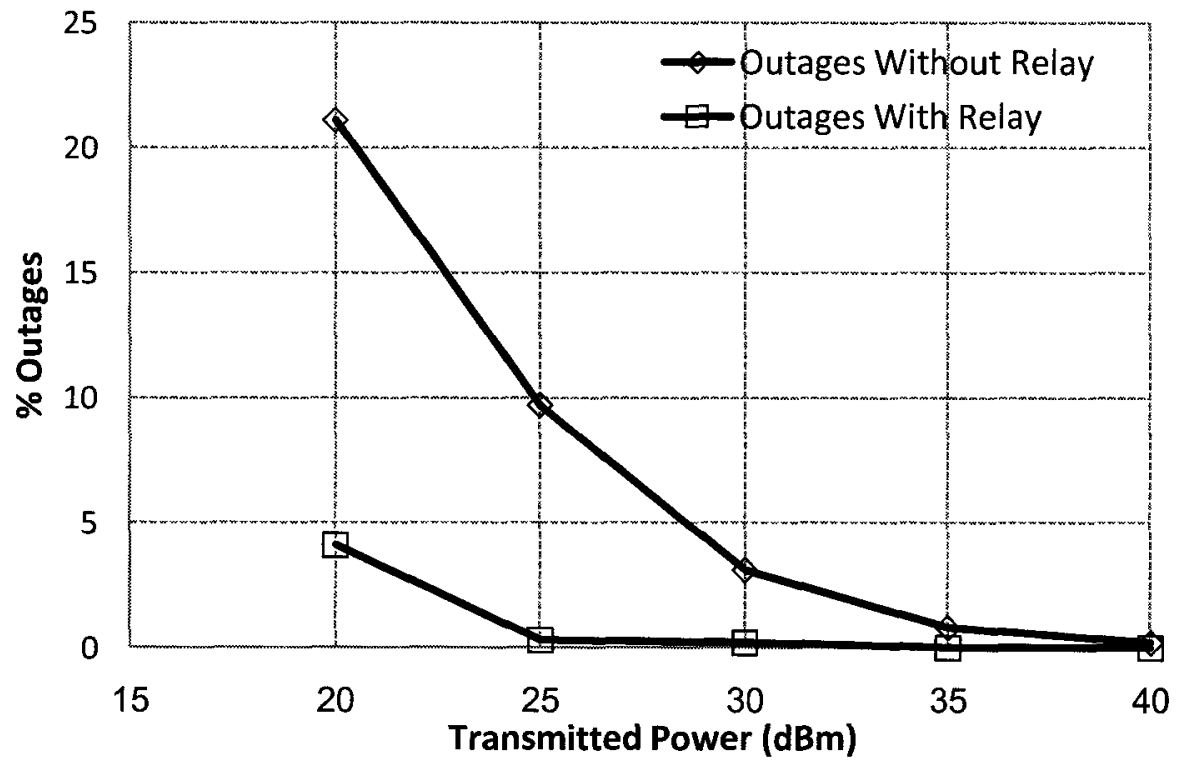

Figure 4.11: Minimum cell outage profile. 


\section{Conclusion and Topics of Future Research}

\subsection{Conclusion}

In this thesis, we explore the use of rateless coding for wireless relay systems. The relay system works based on non-simultaneous TDD relaying, with which the relay and the source transmit in different time slots. The receiver is assumed responsible for the relay association, based on the estimated CSI of the directly connected channels. Three different decode-and-forward relay methods are considered: startover, continue and combine. The startover relaying is the simple cascade multihop relaying. The continue relaying is the method where the relay complements the source transmission by sending additional parity bits instead of resending the whole codeword again as in the startover and combine methods. The combine relaying method is similar to the startover method, except that the receiver combines the data sent by the source with the data sent by the relay.

The theoretical throughput performances were derived for the three relaying modes, and were validated against the simulation results.

The throughput performances of the three relaying modes were generalized to triangular relaying systems. The startover and combine relaying can be improved in the condition that the destination receiver has the full CSI of the three channels: S-D, R-D and S-R. However, the continue relaying method always outperforms the other methods of relaying, while only requiring the CSI of the directly connected channels, and it has 
the least complexity in relay association. It naturally guarantees either the same or better throughput performance than single-hop transmission over all SNRs.

The relaying methods were applied to a linear relay system model, assuming the destination receiver has the CSI of the direct channels S-D and R-D. Both the startover and the combine relaying methods have better throughput performances than the direct transmission for low S-D SNRs, but they perform worse at medium to high S-D SNRs. The combine relaying method has the lowest throughput performance among the three relaying methods, but this may be an artifact of the RC/QC-LDPC code that was used for the simulations. The best relaying method was found to be the continue relaying; even at high S-D SNRS it performs better than or equal to the direct transmission.

The continue relaying method was applied to a single-cell system of three sectors and three relays. The throughputs were enhanced using this method of relaying. The outage probability was reduced vastly at low transmission power levels; better than increasing the transmission power by factor of ten.

\subsection{Future Research Topics}

This research work suggests further research topics that can add more to the wireless communication research community.

The RC/QC-LDPC code that was used performs relatively well on a single-hop transmission. However when applied to relaying systems, it is less effective. This was because data of the same codeword travel over independent channels, experiencing 
different SNRs but are still decoded as one block. LLRs are generated differently for different codeword segments. This raises the question, how do LDPC codes behave if code bits experience different SNR channels? How is this phenomenon related to the structure of LDPC codes? Is it related to the intra-dependency of code bits? This would be an interesting research topic.

Another research topic that should be tackled is the performance of continue relaying when applied to multi-relay network and more specifically ad hoc networks. The theoretical performance of continue relaying may be extended and generalized to multi-relays, yet it has to be verified experimentally.

This research evaluates the downlink channel performance, but not the impact of the uplink channels. A good research topic would be investigating the impact of imperfect uplink channels on rateless coding for relay networks.

The CRC imperfection and overhead impact were ignored in this research. This impact needs to be investigated more with more realistic assumptions.

Although researchers have found that good AWGN codes tend to be good on other channels [48], it is interesting to know how rateless continue relaying method would behave on different channels like multipath fading and others.

This research sought simplicity in resource allocation and relay-association; it was designed based on non-simultaneous relaying. An interesting subject that is a good candidate for further studies is: evaluating the performance of the rateless relaying methods (startover, combine and continue) in simultaneous TDD relaying and full duplex relaying systems. 
The last topic of interest is related to the combine relaying. In theory, combine relaying should behave better than startover. However, the combine relaying method was shown to perform worse than the startover. Also, its performance did not closely match its theoretical performance especially at low SNRs and when the relay is closer to the source. More investigation and study on the combine relaying may reveal much about the relation between practical combining methods and other factors like code structure, LR calculation, and others. 


\section{Appendix}

\section{A RC/QC-LDPC Coding Using 3GPP2}

\section{A.1 3GPP2 QC-LDPC Codes}

The 3GPP2 suggests a series of parity-check matrices based on the length of the information packet size. In general, its base parity-check matrix $\boldsymbol{H}_{B}$ takes the following form:

$$
\boldsymbol{H}_{B}=\left[\begin{array}{cccc}
\boldsymbol{P}_{1,1} & \boldsymbol{P}_{1,2} & \ldots & \boldsymbol{P}_{1, n_{B}} \\
\boldsymbol{P}_{2,1} & \boldsymbol{P}_{2,2} & \because & \boldsymbol{P}_{2, n_{B}} \\
\vdots & \vdots & \ldots & \vdots \\
\boldsymbol{P}_{m_{B}, 1} & \boldsymbol{P}_{m_{B}, 2} & & \boldsymbol{P}_{m_{B}, n_{B}}
\end{array}\right]
$$

where the $\left\{\boldsymbol{P}_{i, j}\right\}$ matrices are the permuted circulants of size $Q \times Q$, chosen to be either

permuted identity matrixes $\left\{\boldsymbol{I}_{Q \times Q}^{(e)}\right\}$ of $e$ permutations, or zero matrices $\left[\mathbf{0}_{Q \times Q}\right]$. The permutation process cyclically shifts every row of $I_{Q \times Q}$ by $e$ places to the left. The mother parity-check matrix $\boldsymbol{H}_{m t h}$ resulted from inserting permuted circulants and zero matrices into $\boldsymbol{H}_{B}$, will look like: [30]

$$
\boldsymbol{H}_{m t h}=\left[\begin{array}{ll}
\boldsymbol{H}_{m t h}^{\prime} & \mathbf{0} \\
\boldsymbol{H}_{e m t h}^{\prime} & \boldsymbol{I}
\end{array}\right]
$$

where $\mathbf{0}$ is a zero matrix, and $\boldsymbol{I}$ is an identity matrix. $\boldsymbol{H}_{m t h}^{\prime}$ is looked at as the original matrix of $\boldsymbol{H}_{m t h}$. On the other hand, $\boldsymbol{H}_{\text {emth }}^{\prime}$ together with $\boldsymbol{I}$ can be thought of as the extended part of $\boldsymbol{H}_{m t h}^{\prime}$. 
The size of $\boldsymbol{H}_{B}$ is $m_{B} \times n_{B}$ of permuted circulants. After inserting the $Q \times Q$ circulants into $\boldsymbol{H}_{B}$, the size of the resulted matrix $\left(\boldsymbol{H}_{m t h}\right)$ is $M_{B} \mathrm{x} N_{B}=\left(m_{B} Q\right) \mathrm{x}\left(n_{B} Q\right) . \boldsymbol{H}_{m t h}^{\prime}$ has a size of $(m Q) \times(n Q)$ with a systematic part of length $K=k_{B} \times Q$. The sizes of the aforementioned matrices depend on the design, and intrinsically depend on the information message size $K$. [45]

The general structure of $\boldsymbol{H}_{m t h}$ is depicted in Figure A.1. $\boldsymbol{H}_{m t h}^{\prime}$ is considered as the basis of $\boldsymbol{H}_{m t h}$ and consists of three parts: $\boldsymbol{H}_{m t h}^{\prime}=[\boldsymbol{A}|\boldsymbol{B}| \boldsymbol{C}]$. The systematic part of $\boldsymbol{H}_{m t h}^{\prime}$ is represented by matrix $\boldsymbol{A}$, and the parity part is represented by $[\boldsymbol{B} \mid \boldsymbol{C}]$. The systematic part $\boldsymbol{A}$ picks different information message bits and relates them to parity check equations. The parity part $[\boldsymbol{B} \mid \boldsymbol{C}]$ complements the parity check equations. Interestingly, the parity part $[\boldsymbol{B} \mid \boldsymbol{C}]$ possesses a modular structure that enables easy encoding. The columns of $C$, are all of weight 2 inspiring us to use "combining" for increasing the code rate. On the other hand, columns in part $\boldsymbol{B}$ are of weight 3 which dictates using puncturing instead. 


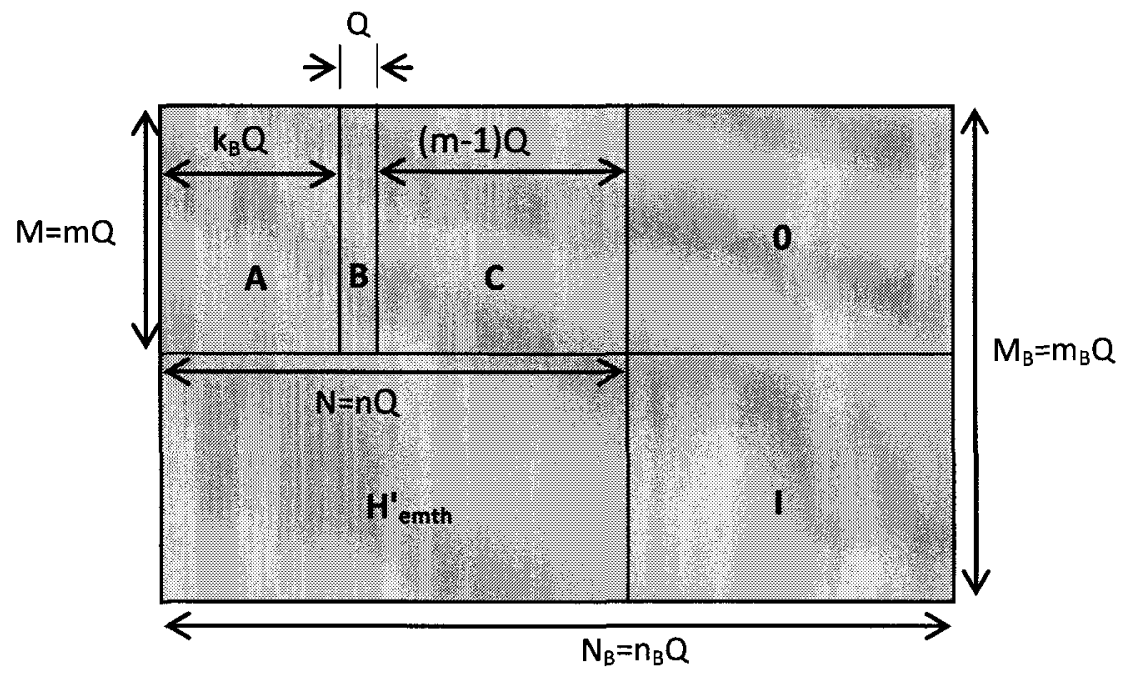

Figure A.1: The general structure of the parity-check-matrix $\boldsymbol{H}_{m t h}$

\section{A.2 RC Code Structure of 3GPP2 QC-LDPC Codes}

The target here is to construct a series family of RC/QC-LDPC parity-check matrices. For higher code rates, we will use the two methods puncturing and combining, while for expanding we will use the method of splitting. Our target code rates are of the range from $R_{c}=0.1$ to 0.96 . The corresponding parity check matrices are the matrices set $\left\{\boldsymbol{H}_{i}\right\}$, with sizes of $\left\{M_{i} \times N_{i}\right\}$, where $\boldsymbol{H}_{0}$ would be the highest rated parity-check matrix.

Noting that the right part of $\boldsymbol{H}_{m t h}^{\prime}$ is of weight 1 , makes it suitable for puncturing as we lose one parity check per each punctured parity node. Puncturing all columns of weight 1 to the right of $\boldsymbol{H}^{\prime}{ }_{m t h}$ and the corresponding rows, leaves us with $\boldsymbol{H}_{m t h}^{\prime}$. The weights of the columns in part $\boldsymbol{C}$ of $\boldsymbol{H}_{m t h}^{\prime}$ are 2, encouraging us to use combining instead of puncturing to prevent losing much of the structure of $\boldsymbol{H}_{m t h}^{\prime}$. Matrix $C$ has a 
special structural feature; the rows that have 1 's in the columns of part $C$ are $Q$-rows apart. The general strategy of structuring the set $\left\{\boldsymbol{H}_{i}\right\}$ from $\boldsymbol{H}_{m t h}$ is as follows:

1. For $N_{i}>N_{B}$ : use splitting to extend the code.

2. For $\left(k_{B}+m\right) \mathrm{x} Q<N_{i}<N_{B}$ : use puncturing to generate higher code rates.

3. For $\left(k_{B}+1\right) \times Q<N_{i}<\left(k_{B}+m\right) \times Q$ : use puncturing and combining to generate higher code rates.

The incremental steps between any parity check matrix $\boldsymbol{H}_{i}$ and the next parity-check matrix $\boldsymbol{H}_{i+1}$ can be made variable or constant. In this work, a constant step that is equal to $L_{\text {inc }}=Q / 2$ is used, to exploit the maximum of rate compatibility and find the optimum code rate $R_{c}(i)$. The following is the a detailed algorithm to build the series family of compatible rated parity-check matrices $\left\{\boldsymbol{H}_{i}\right\}$.

1- For $1 \leq i \leq(2 m-2), \boldsymbol{H}_{i}$ results from the following procedure:

a. Puncture all weight-1 columns and their associated rows:

Columns $V\left(k_{B} Q+j\right) ; m+1 \leq j \leq M_{B}$, where $M_{B}=m_{B} Q$

Rows $R(j) \quad ; m+1 \leq j \leq M_{B}$, where $M_{B}=m_{B} Q$

b. Combine rows: for $j=m Q$, down to $j=\frac{Q}{2}(1+i)+1$

1. $R(j-Q)=R(j) \oplus R(j-Q)$

2. Remove row $R(j)$ and column $V\left(k_{B} Q+j\right)$

2- $\boldsymbol{H}_{0}$ results from puncturing rows $R(j)$ and columns $V\left(k_{B} Q+j\right)$ of $\boldsymbol{H}_{1}$, where $\left(\frac{Q}{2}+\right.$ 1) $\leq j \leq Q$

3- For $i=(2 m-1): \boldsymbol{H}_{i}=\boldsymbol{H}_{m t h}^{\prime}$

4- For $(2 m) \leq i \leq\left(2 m_{B}-2\right)$ 
a. Set $\boldsymbol{H}_{i}=\boldsymbol{H}_{m t h}$

b. Puncture rows $R(j)$ and columns $V\left(k_{B} Q+j\right)$ of $\boldsymbol{H}_{m t h}$; where $\frac{Q}{2}(1+i)+1 \leq$ $j \leq M_{B} ;$ where $M_{B}=m_{B} Q$

5- $\mathrm{H}_{2 \mathrm{~m}_{\mathrm{B}}-1}=\mathrm{H}_{\mathrm{mth}}$

6- For $2 m_{B} \leq i: \boldsymbol{H}_{i}$ is found by extending $\boldsymbol{H}_{i-1}$ as follows:

a. Start with $\boldsymbol{H}_{i}=\boldsymbol{H}_{i-1}$

b. Append a column of zeros to $\boldsymbol{H}_{\boldsymbol{i}}$

c. Find the largest weighted row $R(j)$ in $\boldsymbol{H}_{i}:|R(j)| \geq|R(x)|$, for $x=1 \rightarrow m_{i}=$ $\frac{Q}{2}(1+i)$

d. Split $R(j)$ into $R_{1}$ and $R_{2}$ such that: $R_{1} \cup R_{2}=R(j), R_{1} \cap R_{2}=\emptyset$, and $\left|R_{1}\right|-\left|R_{2}\right| \in\{0,1\}$

e. Replace row $R(j)$ with $R_{1}$, and set the last element in this row to $1\left(H_{i}\left(j, N_{i}\right)=\right.$ 1).

f. Append row $R_{2}$ to $H_{i}$, and set element $H_{i}\left(M_{i}, N_{i}\right)=1$.

g. Repeat steps b-to-f until $N_{i}=N_{\max }$.

By the end of this algorithm, we will have a set of parity-check matrices $\left\{\boldsymbol{H}_{i}\right\}$, of sizes $M_{i}=\frac{Q}{2}(1+i)$ and $N_{i}=k_{B} Q+M_{i}$

\section{A.3 Encoding 3GPP2 RC/QC-LDPC Codes}

As the RC/QC-LDPC encoder receives the CRC-padded information message $\boldsymbol{u}$ of size $K=k_{B} Q$, it encodes it to the lowest rate $R_{c}=0.1$ to yield a codeword $c$ of size $10 K$. The encoding procedure found in the 3GPP2 LDPC proposal is enough to encode up to 
$N_{B}$ bits. The remaining codeword bits must use the extended parity-check matrices $\left\{\boldsymbol{H}_{i}\right\}$ of $\boldsymbol{H}_{m t h}$. The 3GPP2 encoding procedure involves multi-step encoding and matrix inversions.

This research work opts not to use the 3GPP2 encoding procedure. Instead, it uses the parity check matrix set $\left\{\boldsymbol{H}_{i}\right\}$. Note that the code is $\mathrm{RC}$, so each time we generate a parity check block of length $Q / 2$, a different parity check matrix is used. Hence, every parity-check matrix can be used to encode $Q / 2$ parity bits. The complexity of this procedure is low; it is straightforward substitution and mod-2 addition. Codeword bits $c_{1}$-to- $C_{K}$ are the CRC padded information bits. The first $Q / 2$ parity bits are encoded using $\boldsymbol{H}_{0}$, of which the parity part is diagonal of 1-elements. Every successive block of $Q / 2$ parity bits is encoded using the subsequent parity check matrix. This procedure avoids finding inverse matrices.

The general structure of $\boldsymbol{H}_{i}$ looks like:

$$
\boldsymbol{H}_{i}=\left[\begin{array}{cc}
\boldsymbol{H}_{i}^{\prime} & \boldsymbol{H}_{i}^{\prime \prime} \\
\boldsymbol{H}_{i}^{\prime \prime \prime} & \boldsymbol{I}
\end{array}\right]
$$

The second row of matrices are of size $\left(\frac{Q}{2}\right) \times N_{i}$. Actually, this part is all that is required for encoding. The following encoding procedure produces a series of RC codewords $c_{N_{i}}$, each with size $N_{i}$ :

1. Start with the message bits (CRC padded information message) $\boldsymbol{u}$ of size $K=k_{B} Q$.

2. Encode $\boldsymbol{u}$ using $\boldsymbol{H}_{0}: \boldsymbol{H}_{0}=\left[\boldsymbol{H}_{0}^{\prime \prime \prime} \mid \boldsymbol{I}\right]$ to find the first $Q / 2$ parity bits

3. Each time, use the last $Q / 2$ rows of $\boldsymbol{H}_{i}:\left[\boldsymbol{H}_{i}^{\prime \prime \prime} \mid \boldsymbol{I}\right]$ to encode $\boldsymbol{c}_{N_{i-1}}$ to find the next $Q / 2$ parity check bits and append them to $c_{N_{i-1}}$ to yield $\boldsymbol{c}_{N_{i}}$. 
The computation is straightforward; however it requires a proportionally large memory space to store the set of $\left[\boldsymbol{H}_{i}^{\prime \prime \prime} \mid \boldsymbol{I}\right]$ matrices.

\section{A.4 Decoding 3GPP2 RC/QC-LDPC Codes}

In decoding, the method of message-passing algorithm is used. However, we will use the method that was used by Neal [46], where likelihood ratios (LRs) are propagated instead of logarithm likelihood ratios (LLRs). The likelihood ratio $\left(\mathrm{LR}_{n}=p_{n}\right)$ of the $n^{\text {th }}$ bit in codeword $c=\left\{c_{1}, c_{2}, \ldots c_{N_{i}}\right\}$ after observing the corresponding received sequence $\boldsymbol{r}=\left\{r_{1}, r_{2}, \ldots r_{N_{i}}\right\}$, is given as:

$$
\mathrm{LR}_{n}=p_{n}=\frac{\operatorname{Pr}\left[c_{n}=1 \mid \boldsymbol{r}\right]}{\operatorname{Pr}\left[c_{n}=0 \mid \boldsymbol{r}\right]}=\frac{f\left(r_{n} \mid c_{n}=1\right)}{f\left(r_{n} \mid c_{n}=0\right)} \frac{\operatorname{Pr}\left[c_{n}=1 \mid\left\{r_{i \neq n}\right\}\right]}{\operatorname{Pr}\left[c_{n}=0 \mid\left\{r_{i \neq n}\right\}\right]}
$$

provided that codeword bits are independent and $c_{n}$ is equally probable to be zero or one. The term $\mathrm{LR}_{n}^{\text {init }}=p_{n}^{(0)}=\frac{f\left(r_{n} \mid c_{n}=1\right)}{f\left(r_{n} \mid c_{n}=0\right)}$ is the initial value of the likelihood ratio of bit $n$, and it represents the intrinsic information about $c_{n}$. On the other hand, the term $\frac{\operatorname{Pr}\left[c_{n}=1 \mid\left\{r_{i \neq n}\right\}\right]}{\operatorname{Pr}\left[c_{n}=0 \mid\left\{r_{i \neq n}\right\}\right]}$ is the extrinsic information about $c_{n}$ and represents the contribution of other codeword bits. The iterative computation of the likelihood ratio is found using the following equation: [46]

$$
\begin{aligned}
p_{n} & =\mathrm{LR}_{n}^{\text {init }} * \prod_{i \in \mathcal{M}_{n}} f^{-1}\left(\prod_{j \in \mathcal{N}_{m}-n} f\left(p_{i, j}\right)\right) \\
& =\frac{f\left(r_{n} \mid c_{n}=1\right)}{f\left(r_{n} \mid c_{n}=0\right)} \prod_{i \in \mathcal{M}_{n}} f^{-1}\left(\prod_{j \in \mathcal{N}_{m}-n} f\left(p_{i, j}\right)\right)
\end{aligned}
$$


where $f(x)=\frac{1-x}{1+x}$. The term $p_{i, j}$ is the LR propagated from the variable node $j$ to the check node $i . \mathcal{N}_{m}=\left\{n: H_{i}(m, n)=1\right\}$ is the set of $\{n\}$ variable nodes which are involved in the $m^{\text {th }}$ check node, and $\mathcal{M}_{n}=\left\{m: H_{i}(m, n)=1\right\}$ is the set of $\{m\}$ check nodes which are involved in the $n^{\text {th }}$ variable node. The set $\left(\mathcal{N}_{m}-n\right)$ represents a set of variable nodes excluding the $n^{\text {th }}$ variable node. The function $f(x)$ is a special function that is used by the check nodes to combine propagated LRs. It is used here instead of the tanh function used with conventional LDPC decoders. One special property of $f(x)$ is that it is the inverse of its own, i.e. $f^{-1}(f(x))=x$, for $x>-1$.

The hard decision of codeword bits is done by comparing $p_{n}$ with 1 : 1

$p_{n} \gtrless 1$

0

If $p_{n}$ is greater than 1 then $c_{n}$ is 1 , If $p_{n}$ is less than 1 then $c_{n}$ is 0 , and if $p_{n}$ is equal to 1 then $c_{n}$ is equally likely to be 0 or 1.

Since there is a family of parity check matrices $\left\{\boldsymbol{H}_{i}\right\}$ of different sizes $\left\{\left(M_{i}, N_{i}\right)\right\}$, one option of decoding is to use the matching parity check matrix for each codeword $\boldsymbol{r}_{N_{i}}$ being received. The decoding algorithm is:

1. Initialize:
a. $t_{m, n}^{(0)}=1$, for all $m \in\left\{1, \ldots M_{i}\right\}$ and $n \in \mathcal{N}_{m}$
b. $p_{n}^{(0)}=\frac{f\left(r_{n} \mid c_{n}=1\right)}{f\left(r_{n} \mid c_{n}=0\right)}$, for all $n \in\left\{1, \ldots N_{i}\right\}$

2. Iterate for number of iterations: $l=1,2, \ldots l_{\max }$

a. Update check-nodes for $m \in\left\{1, \ldots M_{i}\right\}$ and $n \in \mathcal{N}_{m}$ : 


$$
t_{m, n}^{(l)}=f\left(\prod_{j \in \mathcal{N}_{m}-n} f\left(p_{j}^{(l-1)}\right)\right)
$$

b. Update bit-nodes for $n \in\left\{1, \ldots N_{i}\right\}$ :

$$
p_{n}^{(l)}=p_{n}^{(0)}+\sum_{m \in \mathcal{M}_{n}} t_{m, n}^{(l)}
$$

3. Check for valid codewords:

a. Bit-hard decision $c_{N_{i}}=\left[\begin{array}{lll}c_{1} & \ldots & c_{N_{i}}\end{array}\right]$ :

$$
\begin{aligned}
& \text { - } p_{n}^{(l)}>1 \rightarrow c_{n}=1 \quad \text {; for all } n \in\left\{1, \ldots N_{i}\right\} \\
& \text { - } p_{n}^{(l)}<1 \rightarrow c_{n}=0 \quad \text {; for all } n \in\left\{1, \ldots N_{i}\right\}
\end{aligned}
$$

b. Compute validity:

$$
\text { if } \boldsymbol{c}_{N_{i}} * \boldsymbol{H}_{\boldsymbol{i}}^{\boldsymbol{T}}=0 \text {, quit. Otherwise, iterate more. }
$$

This research work will use this method. One drawback of exactly matching parity check matrices to the codeword lengths is that it creates demand for a large demand for large memory to store every parity check matrix generated. Other options are still valid and might be used successfully, like using only a selected number of check matrices, provided that each matrix used is of the same size as the received codeword or larger. One can always use $\boldsymbol{H}_{i}$ to decode $\boldsymbol{r}_{N_{j}}$, provided $j<i$. If the parity matrix used is larger than the received codeword length, the remaining variable nodes can be initialized with likelihood ratios of value 1 's, or masked out so they don't contribute to the decoding process.

The initialization value $\mathrm{LR}_{n}^{\text {init }}=p_{n}^{(0)}$ (the intrinsic information) is directly found from the received signal sequences provided the receiver knows the channel parameters. The 
intrinsic information depends on the channel and the type of modulation. For BPSK transmission over AWGN channel of noise power equal to $\sigma_{n}^{2}=\frac{N_{0}}{2}$, the initial likelihood ratio is:

$$
\mathrm{LR}_{n}^{\text {init }}=p_{n}^{(0)}=\frac{f\left(r_{n} \mid c_{n}=1\right)}{f\left(r_{n} \mid c_{n}=0\right)}=\exp \left(\frac{2}{\sigma_{n}^{2}} r_{n}\right)
$$

where $\exp ($.$) is the natural exponential function. However, for 16-QAM modulation the$ situation is different. Following the aforementioned constellation mapping, the Ichannel component is used to find the LRs of the first two bits, and the Q-channel component is used to find the LRs of the remaining two bits. If the received 16-QAM signal is $v=\left[v_{I} v_{Q}\right]$ for bits $\left[b_{1} b_{2} b_{3} b_{4}\right]$, then likelihood ratios of bits $b_{1}$ and $b_{2}$ can be found using $v_{I}$, and the likelihood ratios of bits $b_{3}$ and $b_{4}$ can be found using $v_{Q}$. In other words, the LR of $b_{1}$ is:

$$
\begin{aligned}
\mathrm{LR}_{b_{1}}^{\text {init }}=p_{b_{1}}^{(0)}=\frac{\operatorname{Pr}\left[b_{1}=1 \mid v_{I}\right]}{\operatorname{Pr}\left[b_{1}=0 \mid v_{I}\right]}=\frac{\operatorname{Pr}\left[b_{1}=1, b_{2}=0 \mid v_{I}\right]+\operatorname{Pr}\left[b_{1}=1, b_{2}=1 \mid v_{I}\right]}{\operatorname{Pr}\left[b_{1}=0, b_{2}=0 \mid v_{I}\right]+\operatorname{Pr}\left[b_{1}=0, b_{2}=1 \mid v_{I}\right]} \\
=\frac{f\left(v_{I} \mid b_{1}=1, b_{2}=0\right)+f\left(v_{I} \mid b_{1}=1, b_{2}=1\right)}{f\left(v_{I} \mid b_{1}=0, b_{2}=0\right)+f\left(v_{I} \mid b_{1}=0, b_{2}=1\right)} \\
=\frac{\exp \left(\frac{-1}{N_{0}}\left(v_{I}-3 A\right)^{2}\right)+\exp \left(\frac{-1}{N_{0}}\left(v_{I}-A\right)^{2}\right)}{\exp \left(\frac{-1}{N_{0}}\left(v_{I}+3 A\right)^{2}\right)+\exp \left(\frac{-1}{N_{0}}\left(v_{I}+A\right)^{2}\right)}
\end{aligned}
$$

Applying the same procedure to the remaining bits, will yield the following LRs of $b_{2}$, $b_{3}$ and $b_{4}$ respectively: 


$$
\begin{aligned}
& \mathrm{LR}_{b_{2}}^{\text {init }}=p_{b_{2}}^{(0)}=\frac{\exp \left(\frac{-1}{N_{0}}\left(v_{I}+A\right)^{2}\right)+\exp \left(\frac{-1}{N_{0}}\left(v_{I}-A\right)^{2}\right)}{\exp \left(\frac{-1}{N_{0}}\left(v_{I}+3 A\right)^{2}\right)+\exp \left(\frac{-1}{N_{0}}\left(v_{I}-3 A\right)^{2}\right)} \\
& \mathrm{LR}_{b_{3}}^{\text {init }}=p_{b_{3}}^{(0)}=\frac{\exp \left(\frac{-1}{N_{0}}\left(v_{Q}-3 A\right)^{2}\right)+\exp \left(\frac{-1}{N_{0}}\left(v_{Q}-A\right)^{2}\right)}{\exp \left(\frac{-1}{N_{0}}\left(v_{Q}+3 A\right)^{2}\right)+\exp \left(\frac{-1}{N_{0}}\left(v_{Q}+A\right)^{2}\right)} \\
& \operatorname{LR}_{b_{4}}^{\text {init }}=p_{b_{4}}^{(0)}=\frac{\exp \left(\frac{-1}{N_{0}}\left(v_{Q}+A\right)^{2}\right)+\exp \left(\frac{-1}{N_{0}}\left(v_{Q}-A\right)^{2}\right)}{\exp \left(\frac{-1}{N_{0}}\left(v_{Q}+3 A\right)^{2}\right)+\exp \left(\frac{-1}{N_{0}}\left(v_{Q}-3 A\right)^{2}\right)}
\end{aligned}
$$




\section{References}

[1] Abas El Gamal, Thomas M. Cover, "Multiple user information theory," Proceedings of The IEEE, vol. 68, no. 12, pp. 1466-1483, Dec. 1980.

[2] Ralf Pabst, et al. "Relay-based deployment concepts for wireless and mobile broadband radio," IEEE Communications Magazine, pp. 80-89, Sep. 2004.

[3] Halim Yanikomeroglu, "Cellular multihop communications: infrastruture-based relay network architecture for 4G wireless systems," [Online]. Available: http://www.sce.carleton.ca/faculty/yanikomeroglu/Pub/queens04_hy.doc

[4] Özgür Oyman, J. Nicholas Laneman, and Sumeet Sandhu, "Multihop relaying for broadband wireless mesh networks: from theory to practice," IEEE Communications Magazine, vol. 45, no. 11, pp. 116-122, Nov. 2007.

[5] Jing Jiang, John S. Thompson, Peter M. Grant, and Norbert Goertz, "Practicle compress-and-forward cooperation for the classical relay network," 17th European Signal Processing Conference (EUSIPCO 2009), Glasgow, Scotland, pp. 2421-2425, Aug. 24-28, 2009.

[6] Bobak Nazer, and Michael Gastpar, "Compute-and-forward: harnessing interference with structured codes," Proceedings of the IEEE International Symposium on Information Theory (ISIT 2008), Toronto, Canada, pp. 772-776, July 6112008.

[7] J. N. Laneman, "Cooperative diversity in wireless networks: algorithms and architectures," Ph.D. dissertation, Massachusetts Institute of Technology, Cambridge, MA, Aug. 2002.

[8] Mazen O. Hasna, and Mohamed-Slim Alouini, "End-to-end performance of transmission systems with relays over Rayleigh-fading channels," IEEE Transactions on Wireless Communications, vol. 2, no. 6, pp. 1126-1131, Nov. 2003.

[9] Anders Høst-Madsen, and Junshan Zhang, "Capacity bounds and power allocation for wireless relay channels," IEEE Transactions on Information Theory, vol. 51, no. 6 , pp. 2020-2040, Jun. 2005.

[10] E. van der Meulen, "Three-terminal communication channels," Adv. Appl. Probab., vol. 3, pp. 120-154, 1971.

[11] Thomas Cover, and Abbas El Gamal, "Capacity theorems for the relay channel," IEEE Transactions on Information Theory, vol. IT-25, no. 5, pp. 572-584, Sep. 1979.

[12] Michael Gastpar, and Martin Vetterli, "On the capacity of wireless networks: the relay case," in Proc. IEEE Infocom, New York, NJ, USA, vol. 3, pp. 1577-1586, Jun. 2002. 
[13] Gerhard Kramer, Michael Gastpar, and Piyush Gupta, "Capacity theorems for wireless relay channels," in Proc. $41^{\text {st }}$ Annual Allerton Conf. on Comm., Control and Comp., pp. 1074-1083, Oct. 1-3 2003.

[14] Piyush Gupta, and P. R. Kumar, "The capacity of wireless networks," IEEE Transactions on Information Theory, vol. 46, no. 2, pp. 388-404, Mar. 2000.

[15] Jeff Casura, and Yongyi Mao, "Rateless coding and relay networks," IEEE Signal Processing Magazine, pp. 27-35, Sep. 2007.

[16] Neelesh B. Mehta, Vinod Sharma, and Gaurav Bansal, "Queued cooperative wireless networks with rateless codes," IEEE Globecom 2008 Proceedings, New Orleans, Louisiana, USA, pp. 4619-4624.

[17] Azad Ravanshid, Lutz Lampe, and Johannes Huber, "Signal combining for relay transmission with rateless codes," IEEE International Symposium on Information Theory (ISIT 2009), Seoul, Korea, pp. 508-512, June 28 - July 3, 2009.

[18] Xi Liu, and Teng Joon Lim, “ Fountain codes over fading relay channels," IEEE Transactions on Wireless Ccommunications, vol. 8, no. 6, pp. 3278-3287, June 2009.

[19] Andreas F. Molisch, Neelesh B. Mehta, Jonathan S.Yedidia, , and Jin Zhang, "Performance of Fountain codes in collaborative relay networks," IEEE Transactions on Wireless Communications, vol. 6 no. 11, pp. 4108-4119, Nov. 2007.

[20] Yijia (Richard) Fan, Lifeng Lai, Elza Erkip, and H. Vincent Poor, "Rateless coding for MIMO fading channels: performance limits and code construction," IEEE Transactions on Wireless Communications, vol. 9, no. 4, pp. 1288-1292, Apr. 2010.

[21] Youjian (Eugene) Liu, Mahesh K. Varanasi, and Xinming Huang, "Power scheduling for MIMO relay channels employing rateless codes," ICC 2008 Proceedings, pp.44314435, May 2008.

[22] Myung J Lee, Jianliang Zheng, Young-Bae Ko, and Deepesh Man Shrestha, "Emerging standards for wireless mesh technology," IEEE Wireless Communications, vol. 13, pp. 56-63, Apr. 2006.

[23] IEEE806.16 (2010) IEEE Standard for Local and Metropolitan Area Networks - Part 16: Air Interface for Broadband Wireless Access Systems - Advanced Air Interface. [Online]. Available: http://www.ieee802.org/16/pubs/80216m.html, 2010.

[24] 3GPP TR 36.814 V1.1.10(2009-065). 3rd Generation Partnership Project; Technical Specification Group Radio Access Network; Further Advancements for E-UTRA Physical Layer Aspects (Release 9). [Online]. Avaliable: http://www.3gpp.org.

[25] D. Chase, "Code combining -a maximum likelihood decoding approach for combining an arbitrary number of noisy packets," IEEE Transactions Communications, vol. COM-33, no. 5, pp. 385-393, May 1985.

[26] S. Chakraborty, E. Yli-Juuti, and M. Liinaharja, "An ARQ scheme with packet combining," IEEE Comm. Letters, vol. 2, no. 7, pp. 200-202, July 1998. 
[27] J. G. Proakis, Digital Communications, $4^{\text {th }}$ ed. New York, NY: McGraw-Hill Companies, Inc., 2001.

[28] Michael Luby, "LT codes," 43rd Annual IEEE Symposium on Foundations of Computer Science (FOCS'02), Vancouver, BC, Canada, pp. 271-280, Nov. 2002.

[29] Yuanyuan Ma, "Fountain codes and applications to reliable wireless broadcast system," Proceedings of 2006 IEEE Information Theory Workshop (ITW'06), Punta del Este, Uruguay, pp. 66-70, Mar. 2006.

[30] Haoming Li, "A comparison of rateless codes for wireless communication systems," Master's thesis, Carleton University, Ottawa, Ontario, Canada, Aug. 2007.

[31] J. Hagenauer, "Rate-compatible punctured convolutional codes," IEEE Transactions on Communications, vol. 36, no. 4, pp. 389-400, Apr. 1988.

[32] S. Kallel, and D. Haccoun, "Generalized type II hybrid ARQ scheme using punctured convolutional coding," IEEE Transactions, vol. 38, no.11, pp. 1938-1946, Nov. 1990.

[33] J. Li, and H. Imai, "Performance of HARQ protocols with rate compatible turbo codes," International Symposium on Turbo Codes, Brest, France, 1997.

[34] E. Soljanin, N. Varnica, and P. Whiting, "Incremental redundancy hybrid ARQ with LDPC and Raptor codes," IEEE Transactions Information Theory, submitted for publication, Sep. 2005.

[35] M. Yazdani, and A. Banihashemi, "On construction of rate-compatible low-density parity-check codes," IEEE Communications Letters, vol.8, no. 3, pp. 159-161, March 2004.

[36] J. Li, and K. Narayanan, "Rate-compatible low density parity check codes for capacity-approaching ARQ schemes in packet data communications," in Proc. Int. Conference Comm., Internet and Information Technology (CIIT), Virgin Islands, USA, pp. 201-206, Nov. 2002.

[37] T. Okamura, "A hybrid ARQ scheme based on shortened low-density parity-check codes," IEEE Wireless Comm. \& Networking Conference (WCNC 2008) Proceedings, pp. 82-87, April-March 2008.

[38] T. J. Richardson, M. Amin Shokrollahi, and R. L. Urbanke, "Design of capacityapproaching irregular low-density parity-check codes," IEEE Transactions Information Theory, vol. 47, pp. 657-670, Feb. 2001.

[39] S.-Y. Chung, D. Forney, T. Richardson, R. Urbanke, "On the Design of Low-Density Parity-Check Codes within $0.0045 \mathrm{~dB}$ of the Shannon Limit," IEEE Comm. Letters, vol. 5, no. 2, pp. 58-60, Feb. 2001.

[40] Y. Y. Tai, L. Lan, L. Zeng, S. Lin, L. Fellow, and K. A. S. Abdel-Ghaffar, "Algebraic construction of quasi-cyclic LDPC codes for the AWGN and erasure channels," IEEE Trans. on Communications, vol.54, no.10, pp. 1765-1774, Oct. 2006. 
[41] D. J. C MacKay, Information Theory, Inference and Learning Algorithms, $1^{\text {st }}$ edition Cambridge, England: Cambridge University Press, 2002.

[42] G. Liva, S. Song, L. Lan, Y. Zhang, S. Lin, W. Ryan, "Design of LDPC codes: a survey and new results," to appear, J. Commun. Software and Systems, vol.2, no. 3, pp. 191-211, Sep. 2006.

[43] L. Lan, L.-Q. Zeng, Y. Y. Tai, S. Lin and K. Abdel-Ghaffar, "Constructions of quasi-cyclic LDPC codes for the AWGN and binary erasure channels: a finite fields approach," IEEE Transactions Information Theory, vol. 53, no. 7, pp. 2429-2458, July 2007.

[44] L. Chen, J. Xu, I. Djurdjevic, and S. Lin, "Near-Shannon-limit quasi-cyclic low-density parity-check codes," IEEE Transactions on Communications, vol. 52, no. 7, pp.10381042, July 2004.

[45] 3GPP2. (2007) C.p0084-001-0 v1.088 Physical Layer for Ultra Mobile Broadband (UMB) Air Interface Specification (_c.p0084-001 v1.088 phy clean.pdf). [Online]. Available:http://www.3gpp2.org/Public_html/Misc/C.P0084001_v1.088_PHY_clean _V_Due_9_Aug-2007.pdf

[46] Radford M. Neal, "Software for low density parity check codes," [Online]. Available: http://www.cs.toronto.edu/ radford/ftp/LDPC-2006-02-08/index.html, 2006.

[47] M. Good, and F. R. Kschischang, "Incremental redundancy via check splitting," $23^{\text {rd }}$ Biennial Symposium on Communications, Kingston, Canada, pp. 55-8, May 2006.

[48] M. Franceschini, G. Ferrari, and R. Raheli, "Does the performance of LDPC codes depend on the channel ," IEEE Transactions on Communications, vol. 54, no. 12, pp. 2129-2132, Dec. 2006. 